\title{
Options for the Tevatron Tunnel
}

\author{
December 23, 1993
}

\author{
A Report Prepared for the \\ Fermilab Accelerator Division Head
}

Options for the Tevatron Tunnel, December 23, 1993 Page 1 


\section{I.1 Introduction}

This report was written for the Accelerator Division head to present several accelerator options for using the Tevatron tunnel in the Main Injector era. At this point, few ideas were rejected, and it is recognized that many of these options will be eliminated by imposing non-technical but real constraints (such as funding) or by choosing not to take the technical risks associated with some of the options.

Some options use protons on antiprotons in a collider, others use protons on protons in a collider. Some options retain the Tevatron as a fixed target machine, others replace it.

\section{L.1.1 Structure of This Report}

Section 1 presents a proton antiproton accelerator to replace the Tevatron and run at the highest energy achievable. Since this is the first option considered, this section develops many of the issues used in the later sections as well.

Section 2 presents a proton proton accelerator based on an LHC style 2-in-1 dipole.

Section 3 presents a proton proton accelerator using the Tevatron as one of the accelerators.

Section 4 presents a proton proton accelerator based on SSC dipoles.

Section 5 presents some issues related to the injectors for these accelerators.

Section 6 presents some programmatic issues which, once addressed by the Director, will help eliminate most of these options. This section also presents options based on 13 Tesla dipoles.

\section{L.1.2 Summary of Options}

Table 1 gives a comparison of parameters for the various accelerator options. Table $1 \mathrm{a}$ gives a comparison of various proton 
antiproton colliders. Table $1 \mathrm{~b}$ gives a comparison of various proton proton colliders. Table 1c gives a comparison of various proton proton options based on 13 Tesla dipoles.

The inelastic cross sections used in Tables $1 \mathrm{a}, 1 \mathrm{~b}$ and $1 \mathrm{c}$ are based on extrapolations from present measurements ${ }^{1}$.

Table 1a.

\author{
Parameters for antiproton proton \\ options \\ compared to the Main Injector
}

\begin{tabular}{|c|c|c|c|c|c|}
\hline & $\begin{array}{r}\text { Main } \\
\text { Injector }\end{array}$ & PA4 & PA6 & PAZ & PA8 \\
\hline Protons/bunch & $3.30 \mathrm{E}+11$ & $2.70 \mathrm{E}+11$ & $2.70 E+11$ & $2.70 \mathrm{E}+11$ & $2.70 \mathrm{E}+11$ \\
\hline Pbars/bunch & $3.60 \mathrm{E}+10$ & $1.80 \mathrm{E}+10$ & $1.80 \mathrm{E}+10$ & $1.80 \mathrm{E}+10$ & $1.80 \mathrm{E}+10$ \\
\hline Proton emittance & 25 & 25 & 25 & 25 & $25 \mathrm{~mm}-\mathrm{mr}$ \\
\hline Pbar emittance & 15 & 15 & 15 & 15 & $15 \mathrm{~mm}-\mathrm{mr}$ \\
\hline Beta @IP & 0.25 & 0.25 & 0.25 & 0.25 & $0.25 \mathrm{~m}$ \\
\hline Energy & 1000 & 1000 & 1500 & 1750 & $2000 \mathrm{GeV}$ \\
\hline Bunches & 36 & 72 & 72 & 72 & 72 \\
\hline Bunch length (rms) & 0.6 & 0.35 & 0.32 & 0.31 & $0.3 \mathrm{~m}$ \\
\hline Form Factor & 0.49 & 0.66 & 0.69 & 0.70 & 0.71 \\
\hline Arc Dipole Field & 4.4 & 4.4 & 6.6 & 7.7 & 8.8 Tesla \\
\hline Rev. Frequency & 47.713 & 47.713 & 47.713 & 47.713 & $47.713 \mathrm{kHz}$ \\
\hline Typical Luminosity* & $1.01 E+32$ & $1.12 E+32$ & $1.75 E+32$ & $2.07 E+32$ & $2.40 \mathrm{E}+32 \mathrm{~cm}-2 \mathrm{sec}-1$ \\
\hline Best Luminosity ${ }^{\star \star}$ & $1.50 \mathrm{E}+32$ & $1.68 \mathrm{E}+32$ & $2.62 E+32$ & $3.10 \mathrm{E}+32$ & $3.60 \mathrm{E}+32 \mathrm{~cm}-2 \mathrm{sec}-1$ \\
\hline Integrated Luminosity & 20.44 & 22.57 & 35.3 & 41.7 & $48.4 \mathrm{pb}-1 /$ week \\
\hline Coalescing & 2.5 & 7.5 & 7.5 & 7.5 & $7.5 \mathrm{MHz}$ \\
\hline Bunch Spacing & 396 & 132 & 132 & 132 & $132 \mathrm{nsec}$ \\
\hline Interactions/crossing & 2.66 & 1.47 & 2.39 & 2.89 & 3.49 \\
\hline Inelastic Cross Section & 45 & 45 & 47 & 48 & 50 mbarns \\
\hline Antiproton tune shift & 0.019 & 0.016 & 0.016 & 0.016 & 0.016 \\
\hline Proton tune shift & 0.004 & 0.002 & 0.002 & 0.002 & 0.002 \\
\hline
\end{tabular}

1 Stan Pruss, private communication.

Options for the Tevatron Tunnel, December 23, 1993 Page 3 
Table 1 b.

Note:

For Main Injector the second proton

line is for pbars.

Protons/bunch

Protons/bunch

Proton emittance

Proton emittance

Beta @ IP

Energy

Bunches

Bunch length (rms)

Form Factor

Arc Dipole Field

Rev. Frequency

Typical Luminosity*

Best Luminosity ${ }^{\star \star}$

Integrated Luminosity

Coalescing

Bunch Spacing

Interactions/crossing

Inelastic Cross Section

Proton tune shift

Proton tune shift
Parameters for proton proton options

compared to the Main Injector
Main

Injector

$3.30 \mathrm{E}+11$

$3.60 \mathrm{E}+10$

25

15

0.25

1000

36

0.6

0.49

4.4

47.713

$1.01 \mathrm{E}+32$

$1.50 \mathrm{E}+32$

20.44

$\begin{array}{rrrc}\text { PP4a } & \text { PP4b } & \text { PP6 } & \text { PP8 } \\ & & & \\ 6.00 \mathrm{E}+10 & 2.70 \mathrm{E}+11 & 6.00 \mathrm{E}+10 & 6.00 \mathrm{E}+10 \\ 6.00 \mathrm{E}+10 & 2.70 \mathrm{E}+11 & 6.00 \mathrm{E}+10 & 6.00 \mathrm{E}+10 \\ 25 & 25 & 25 & 25 \mathrm{~mm}-\mathrm{mr} \\ 25 & 25 & 25 & 25 \mathrm{~mm}-\mathrm{mr} \\ 0.25 & 0.25 & 0.25 & 0.25 \mathrm{~m} \\ 1000 & 1000 & 1500 & 2000 \mathrm{GeV} \\ 756 & 108 & 756 & 756 \\ 0.1 & 0.35 & 0.1 & 0.09 \mathrm{~m} \\ 0.93 & 0.66 & 0.93 & 0.94\end{array}$

$4.4 \quad 6.6$

$47.713 \quad 47.713$

$2.02 \mathrm{E}+33 \quad 1.48 \mathrm{E}+33$

$3.02 \mathrm{E}+33 \quad 2.22 \mathrm{E}+33$

406

8.8 Tesla

$47.713 \quad 47.713 \mathrm{kHz}$

$9.88 \mathrm{E}+32$

199

299
$2.00 \mathrm{E}+33 \mathrm{~cm}-2 \mathrm{sec}-1$

$3.00 \mathrm{E}+33 \mathrm{~cm}-2 \mathrm{sec}-1$

$403 \mathrm{pb}-1 /$ week
2.5 None

396

2.66

45

0.019

0.004
7.5 None

132

17.60

45

0.016

0.016
None $\quad \mathrm{MHz}$

18.83

1.93

47

$18.83 \mathrm{nsec}$

2.77

50 mbarns

0.004

0.004

0.004

0.004

*"Typical Luminosity" is the luminosity expected at the beginning of a store.

*"Typical Luminosity" translates to integrated luminosity with a 33\% duty factor.

**"Best Luminosity" means the experiments should be prepared for this on good stores. 
Table 1c.

Note:

For Main Injector the second proton line is for pbars.

Protons/bunch

Protons/bunch

Proton emittance

Proton emittance

Beta@IP

Energy

Bunches

Bunch length (rms)

Form Factor

Arc Dipole Field

Rev. Frequency

Typical Luminosity*

Best Luminosity ${ }^{\star \star}$

Integrated Luminosity

Coalescing

Bunch Spacing

Interactions/crossing

Inelastic Cross Section

Proton tune shift

Proton tune shift
Parameters for proton proton options using 13 Tesla dipoles or a new tunnel compared to the Main Injector and LHC

$\begin{array}{rrrrc}\begin{array}{r}\text { Main } \\ \text { Intector }\end{array} & \text { PP13a } & \text { PP13b } & \text { PP13c } & \text { LHC } \\ 3.30 \mathrm{E}+11 & 6.00 \mathrm{E}+10 & 6.00 \mathrm{E}+10 & 2.00 \mathrm{E}+10 & 1.00 \mathrm{E}+11 \\ 3.60 \mathrm{E}+10 & 6.00 \mathrm{E}+10 & 6.00 \mathrm{E}+10 & 2.00 \mathrm{E}+10 & 1.00 \mathrm{E}+11 \\ 25 & 25 & 25 & 25 & 22 \mathrm{~mm}-\mathrm{mr} \\ 15 & 25 & 25 & 25 & 22 \mathrm{~mm}-\mathrm{mr} \\ 0.25 & 0.25 & 0.25 & 0.25 & 0.5 \mathrm{~m} \\ 1000 & 3000 & 6750 & 6750 & 7000 \mathrm{GeV} \\ 36 & 756 & 2398 & 2398 & 2835 \\ 0.6 & 0.1 & 0.1 & 0.1 & 0.09 \mathrm{~m} \\ 0.49 & 0.93 & 0.93 & 0.93 & 1.00 \\ 4.4 & 4.4 & 13.0 & 13.0 & 8.0 \mathrm{Tesla} \\ 47.713 & 47.713 & 21.206 & 21.206 & 11.111 \mathrm{kHz} \\ 1.01 \mathrm{E}+32 & 2.96 \mathrm{E}+33 & 9.40 \mathrm{E}+33 & 1.04 \mathrm{E}+33 & 1.02 \mathrm{E}+34 \mathrm{~cm}-2 \mathrm{sec}-1 \\ 1.50 \mathrm{E}+32 & 4.45 \mathrm{E}+33 & 1.41 \mathrm{E}+34 & 1.57 \mathrm{E}+33 & 1.53 \mathrm{E}+34 \mathrm{~cm}-2 \mathrm{sec}-1 \\ 20.45 & 597 & 1895 & 211 & 2057 \mathrm{pb}-1 / \text { week }\end{array}$

*"Typical Luminosity" is the luminosity expected at the beginning of a store.

*"Typical Luminosity" translates to integrated luminosity with a 33\% duty factor.

**"Best Luminosity" means the experiments should be prepared for this on good stores.

\section{I.2 Overall Assumptions and Tunnel Configurations}

These assumptions are imposed for the purposes of this report to provide some constraints to allow the report to be completed within a month. They should be revisited before a proper design of any one of the options is pursued further.

\section{L.2.1 Lattice}

Options for the Tevatron Tunnel, December 23, 1993 Page 5 
The concrete walls of the Tevatron tunnel will probably force one to use a lattice very similar to the Tevatron ${ }^{2}$. That is, the dipole lattice of the tunnel arc sections needs to be essentially the same as the present main ring and Tevatron lattices in order to keep the orbit within the confines of the tunnel. In the options presented here, each new dipole will have twice the effective magnetic length of a Tevatron dipole and will replace two Tevatron dipoles in such a way as to equal the bend angle and effective bend center of the replaced dipoles. A particular accelerator option which does not use this constraint cannot be taken seriously until it has successfully accommodated the tunnel cross sections throughout the arcs, including the 48 and 17 locations in addition to the long straight sections.

The lattice quadrupoles of the Tevatron lattice have a gradient of $0.76 \mathrm{~T} / \mathrm{cm}$. The presently installed low beta quadrupoles have a $7.5 \mathrm{~cm}$ coil aperture and a gradient of $1.4 \mathrm{~T} / \mathrm{cm}$ at $4.2 \mathrm{~K}$ which is sufficient to equal the Tevatron's tune up to a proton energy of 2 $\mathrm{TeV}$ if the quadrupoles are constructed with a magnetic length equal to the length of the Tevatron lattice quadrupoles ( $66 \mathrm{in}$.). However, the quadrupole lattice might be improved by using quadrupoles with higher gradients or longer length, made possible by small reductions in the length of the new lattice dipoles.

It is assumed that the low beta arrangement is the same as it is today but capable 3 of providing a $25 \mathrm{~cm} \beta^{*}$. In the options which use protons on antiprotons, the separators are assumed to be in their present locations. For a proton energy of $2 \mathrm{TeV}$, the present low beta lattice would require a gradient of $2.8 \mathrm{~T} / \mathrm{cm}$. This is probably possible by reducing the coil aperture to $6 \mathrm{~cm}$, increasing the cable width approximately $20 \%$ and operating at $1.8 \mathrm{~K}$. An alternative is a modified low beta lattice that reduces the required peak gradient.

\section{L.2.3 Tunnel Figures}

Several figures labeled $\mathrm{MSD}^{4}$ are attached to this report.

2 The Tevatron lattice is very similar to the Main Ring lattice for this same concrete reason.

3 It is assumed that a $25 \mathrm{~cm} \beta^{\star}$ will be made operational in Run Ib.

4 Provided by the Mechanical Support Department. 
Figure MSD1 shows two 12.5 meter dipoles replacing 4 Tevatron dipoles per half cell. The figure also shows that the new quads would be shorter than the existing Tevatron lattice quads by about a factor of two.

Figures MSD2 and 3 demonstrate that 12.5 meter magnets can be inserted into the tunnel through the existing accesses. The magnets must be tilted to make it in through the DO access hatch as shown in MSD2a. The magnets will not fit through the A0 access hatch unless a removable piece of the floor is in fact removed as shown in MSD2b. MSD2c and 2d show the plan view of the required maneuvers at the DO and $\mathrm{AO}$ hatches. The magnets can (barely) fit around the major vehicle accesses as shown in MSD3a. The magnets can (again barely) make it around the DO and BO bypasses as shown in MSD3b and 3c.

The ten figures MSD4a through $4 \mathrm{j}$ show various arrangements of accelerators in F-sector. The following table summarizes these arrangements. For each option, the beams are brought into collision at some elevation relative to the present Tevatron. The amount of this change in elevation is indicated in the table. In one case the change is as little as 0.313 inch, and for another it as much as 21.688 inches. 


\begin{tabular}{|c|c|c|}
\hline & $\begin{array}{l}\text { Change in } \\
\text { Collision }\end{array}$ & \\
\hline Figure & $\begin{array}{l}\text { Elevation } \\
\text { (inches) }\end{array}$ & Option \\
\hline MSD4a & 0.313 & $\begin{array}{l}\text { Flipped SSC dipoles replacing } \\
\text { Tevatron dipoles }\end{array}$ \\
\hline MSD4b & 8.063 & HEB dipoles side by side \\
\hline MSD4c & 8.792 & 2-in-1 LHC style \\
\hline MSD4d & 13.522 & $\begin{array}{l}\text { HEB dipoles over } \\
\text { Tevatron dipoles }\end{array}$ \\
\hline MSD4e & 17.25 & $\begin{array}{l}\text { SSC dipoles over } \\
\text { Tevatron dipoles }\end{array}$ \\
\hline MSD4f & 13.063 & $\begin{array}{l}\text { Flipped SSC dipoles over } \\
\text { Tevatron dipoles }\end{array}$ \\
\hline MSD4g & 15.217 & $\begin{array}{l}\text { Tevatron dipoles over } \\
\text { Flipped SSC dipoles }\end{array}$ \\
\hline MSD4h & 19.902 & $\begin{array}{l}\text { Tevatron dipoles over } \\
\text { HEB dipoles }\end{array}$ \\
\hline MSD4i & 21.688 & $\begin{array}{l}\text { HEB dipoles over } \\
\text { HEB dipoles }\end{array}$ \\
\hline MSD4j & 19.563 & $\begin{array}{l}\text { Flipped SSC dipoles over } \\
\text { SSC dipoles }\end{array}$ \\
\hline
\end{tabular}

\section{I.2.4 Civil Construction}

None of these options require civil construction.

\section{L.2.5 Detectors} inches.

It is assumed that the detectors can be moved up about 12

\section{I.3 Physics Motivation}

One view of the physics motivation for a hadron collider which is the next step after the Tevatron is provided in the Attachment "Physics Sensitivity of Fermilab Machine Options", December 1993 by Christopher T. Hill and Stephen J. Parke. They suggest three 
directions: 1) Top Quark Factory, 2) Higgs Boson, and 3) B-Physics. An antiproton proton collider has a definite advantage for providing a top quark factory in the center of mass energy range up to several $\mathrm{TeV}$ as in the accelerator options described here. The Higgs boson physics needs raw integrated luminosity more than anything else, but an antiproton proton machine may also have an advantage starting at $\mathrm{mH}=60 \mathrm{GeV}$ (the present lower limit) up to about 200 $\mathrm{GeV}$. Finally, hadron colliders offer several clear advantages over $\mathrm{e}^{+} \mathrm{e}^{-}$machines, among which are the ability to produce all B states rather than a few.

\section{L3.1 Top Production Rates}

Figure PH shows a plot ${ }^{5}$ by Steve Parke and Chris Hill showing top quark pair production rates. The figure demonstrates that the cross section for top production is larger for antiproton proton collisions. Tables $2 \mathrm{a}$ and $2 \mathrm{~b}$ provide a comparison of top pair production for the accelerator options discussed in this report.

5 Presented by Chris Hill at the Luminosity Workshop held at Pheasant Run, December 1993. 
Table 2a.

\begin{tabular}{|c|c|c|c|c|}
\hline Injector & PA4 & PA6 & PA7 & PA 8 \\
\hline 409 & 451 & 2,116 & 3,546 & 5,319 tops/week \\
\hline 39 & 43 & 243 & 417 & 725 tops/week \\
\hline 1.0 & 1.10 & 5.2 & 8.7 & $13.0140 \mathrm{GeV}$ \\
\hline 1.0 & 1.10 & 6.3 & 10.7 & $18.7200 \mathrm{GeV}$ \\
\hline
\end{tabular}

$140 \mathrm{GeV}$ top mass

$200 \mathrm{GeV}$ top mass

Tops compared

to Main Injector

1.0

6.3

10.7

$18.7200 \mathrm{GeV}$

The above numbers are derived from the following table based on Hill / Parke.

$140 \mathrm{GeV}$ Tops / pb-1

$200 \mathrm{GeV}$ Tops / pb-1

Top Cross Sections for top mass of $140 \mathrm{GeV}$

top mass of $200 \mathrm{GeV}$

top mass of $140 \mathrm{GeV}$

top mass of $200 \mathrm{GeV}$

Table 2b.

$\begin{array}{rrrrr}20 & 20 & 60 & 85 & 110 \\ 1.9 & 1.9 & 6.9 & 10 & 15\end{array}$

$\begin{array}{rrrr}20.0 & 20.0 & 60.0 & 85.0 \\ 1.9 & 1.9 & 6.9 & 10.0\end{array}$

$110.0 \mathrm{pb}$ for $\mathrm{p}$ pbar $15.0 \mathrm{pb}$ for $\mathrm{p}$ pbar

$p b$ for $p p$ $p b$ for $p p$
$140 \mathrm{GeV}$ top mass

$200 \mathrm{GeV}$ top mass

Tops compared

to Main Injector

Mal
Injecto
409
1.

Top production for proton proton options

compared to the Main Injector

Maln

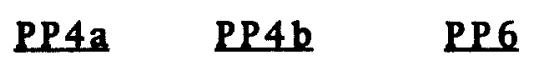

409

39

1.0

1.0
1,792 100

3,656

203

4.4

2.6
8.9

5.2
11,947

1,075

29.2

27.7
PP 8

36,254 tops/week 4,028 tops/week

$88.7140 \mathrm{GeV}$ $103.7200 \mathrm{GeV}$

The above numbers are derived from the following table based on Hill / Parke.

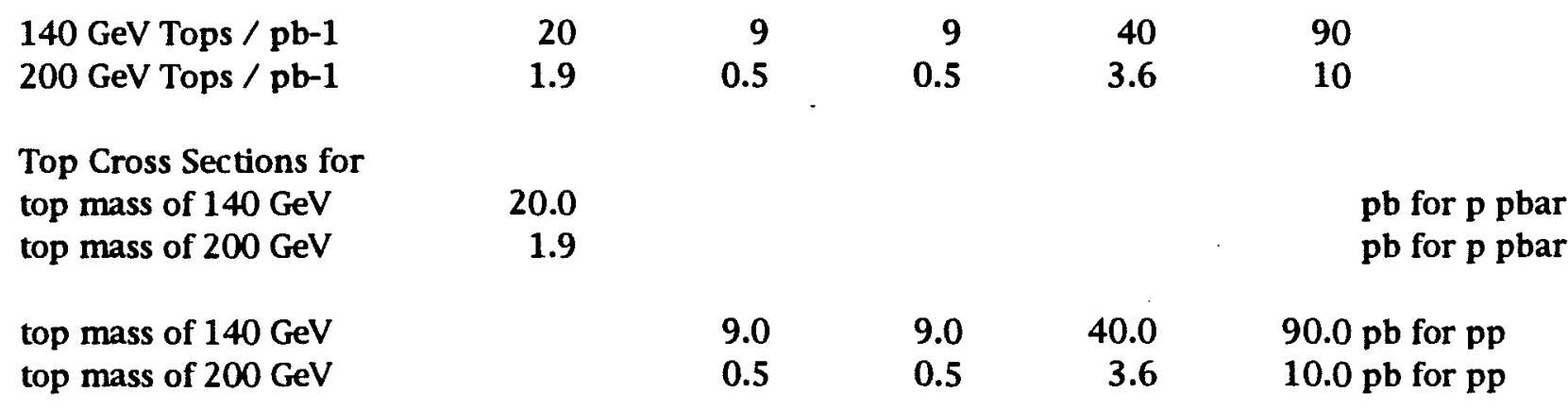

Options for the Tevatron Tunnel, December 23, 1993 Page 10 
Table 2c.

$140 \mathrm{GeV}$ top mass

$200 \mathrm{GeV}$ top mass

Tops compared

to Main Injector

\section{Main}

Top production for proton proton options

using 13 Tesla dipoles or a new tunnel

compared to the Main Injector and LHC
Injector PP13a PP13b PP13c LHC

$409 \quad 179,247 \quad 6,633,238 \quad 737,026 \quad 7,405,389$ tops/week

$39 \quad 23,900 \quad 871,797 \quad 96,866 \quad 987,385$ tops/week

$\begin{array}{lllll}1.0 & 438 & 16226 & 1803 & 18115140 \mathrm{GeV}\end{array}$

The above numbers are derived from the following table based on Hill / Parke. Note: PP13b, PP13c and LHC are estimates.

$\begin{array}{lrrrrr}140 \mathrm{GeV} \text { Tops } / \mathrm{pb}-1 & 20 & 300 & 3500 & 3500 & 3600 \\ 200 \mathrm{GeV} \text { Tops } / \mathrm{pb}-1 & 1.9 & 40 & 460 & 460 & 480\end{array}$

Top Cross Sections for top mass of $140 \mathrm{GeV}$ top mass of $200 \mathrm{GeV}$

top mass of $140 \mathrm{GeV}$ top mass of $200 \mathrm{GeV}$

$\begin{array}{rrrr}300.0 & 3500.0 & 3500.0 & 3600.0 \mathrm{pb} \text { for } \mathrm{pp} \\ 40.0 & 460.0 & 460.0 & 480.0 \mathrm{pb} \text { for } \mathrm{pp}\end{array}$

\section{I.4 Luminosity Lifetime and Integrated Luminosities}

The top production rates in Table 2 are calculated from the weekly integrated luminosity from Table 1 . The weekly integrated luminosity is calculated by assuming

$$
\int_{0}^{1 \text { week }} L d t=L_{\text {typical }}(168 \text { hours } / 3)
$$

The factor of 3 includes loss of luminosity due to luminosity lifetime effects, the time it takes to refresh the store, and unscheduled down time. This relationship is a reasonable approximation to the realities during the previous collider runs. 
However, one can calculate 6 the particle loss due to collisions and vacuum, the transverse emittance growth due to intrabeam scattering and vacuum, and the longitudinal emittance growth due to intrabeam scattering. Then, accounting for the particle losses and emittance growths, one can calculate both the luminosity lifetime and the integrated luminosity. This has been done in Table 3 where the initial luminosity lifetime is displayed along with the integrated luminosity assuming a 15 hour store.

There are two sets of lifetime and integrated luminosity displayed in Table 3: one assuming only the inelastic cross section leads to particle loss due to collisions, and the other assuming the total cross section leads to particle loss. The differences in integrated luminosities is not very large.

The relationship I.4.1 assumes that the ratio of integrated luminosity to the typical luminosity is a constant. This is, of course not true, since the lifetimes are quite different. The options associated with the poorest lifetimes will give overestimates of the integrated luminosity using I.4.1, and the options with the better lifetimes will be underestimated. Nevertheless, for the actual options shown, this is less than a $40 \%$ effect in the worst case.

Table 3a.

$\begin{array}{lllll}\text { Main } & \text { Initial Lifetime } & \text { and } & \text { Integrated Luminosity } \\ \text { Injector } & \text { PA4 } & \text { PA6 } & \text { PA7 } & \text { PA8 }\end{array}$

Typical Luminosity*

Initial Luminosity**

Initial lifetime

15 hour integrated ${ }^{\star \star}$

Initial lifetime ${ }^{\star \star \star}$

15 hour integrated ${ }^{\star \star \star}$

Total Cross Section
$1.01 \mathrm{E}+32$

$1.14 \mathrm{E}+32$

$1.12 \mathrm{E}+32$

$1.04 \mathrm{E}+32$

$1.75 \mathrm{E}+32$

$2.01 \mathrm{E}+32$

$2.07 \mathrm{E}+32$

$2.20 \mathrm{E}+32$

$2.40 \mathrm{E}+32 \mathrm{~cm}-2 \mathrm{sec}-1$

$2.94 \mathrm{E}+32 \mathrm{~cm}-2 \mathrm{sec}-1$
10.66

3.75

9.22

3.49

75
10.9

3.5

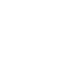

75
5.1

5.3

5.7

5.6

5.1

5.2

86

88
5.1 hours

$6.7 \mathrm{pb}-1$

4.9 hours

$5.8 \mathrm{pb}-1$

90 mbarns

*This is the value from the parameters table

* This value is calculated from an overlap integral program using the inelastic cross section

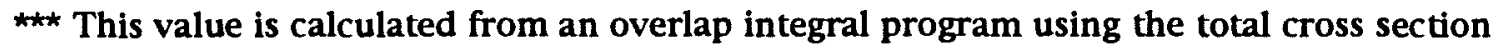

6 Calculations provided by S. Pruss based on TM-1646 "Intrabeam Scattering in the Tevatron Collider Upgrade" by David Finley, and "Calculations of Integrated Luminosity for Beams Stored in the Tevatron Collider" by D. A. Finley in Proceedings of the 1989 IEEE Particle Accelerator Conference, Chicago, IL. 
Table 3b.

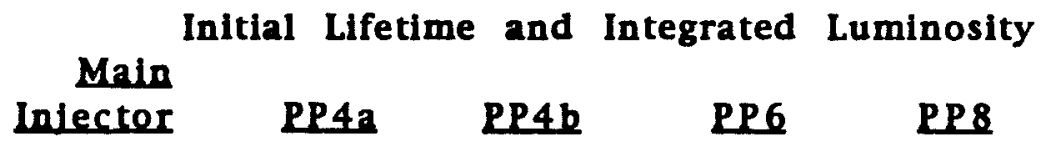

$\begin{array}{lrrrrc}\begin{array}{l}\text { Typical Luminosity* } \\ \text { Initial Luminosity }\end{array} & 1.01 \mathrm{E}+32 & 9.88 \mathrm{E}+32 & 2.02 \mathrm{E}+33 & 1.48 \mathrm{E}+33 & 2.00 \mathrm{E}+33 \mathrm{~cm}-2 \mathrm{sec}-1 \\ & 1.14 \mathrm{E}+32 & 1.07 \mathrm{E}+33 & 2.13 \mathrm{E}+33 & 1.67 \mathrm{E}+33 & 2.27 \mathrm{E}+33 \mathrm{~cm}-2 \mathrm{sec}-1 \\ \begin{array}{l}\text { Initial lifetime } \\ 15 \text { hour integrated }\end{array} & 10.66 & 15 & 5.0 & 13 & 12 \text { hours } \\ & 3.75 & 42 & 57 & 61 & 80 \mathrm{pb}-1 \\ \text { Initial lifetime } & & & & & \text { hours } \\ 15 \text { hour integrated } & 9.22 & & 4.51 & & \text { pb-1 } \\ \text { Total Cross Section } & 3.49 & & 53 & 86 & 90 \text { mbarns }\end{array}$

*This is the value from the parameters table

** This value is calculated from an overlap integral program using the inelastic cross section *** This value is calculated from an overlap integral program using the total cross section

Table 3c.

\begin{tabular}{|c|c|c|c|c|c|}
\hline Table $3 c$ & Main & nitial Life & ime and & Integrated & Luminosity \\
\hline & Injector & PP13a & PP13b & PP13C & LHC \\
\hline Typical Luminosity* & $1.01 \mathrm{E}+32$ & $2.96 E+33$ & $9.40 \mathrm{E}+33$ & $1.04 \mathrm{E}+33$ & $1.02 \mathrm{E}+34 \mathrm{~cm}-2 \mathrm{sec}-1$ \\
\hline Initial Luminosity ${ }^{\star \star}$ & $1.14 \mathrm{E}+32$ & $2.98 \mathrm{E}+33$ & $9.55 E+33$ & $1.06 \mathrm{E}+33$ & $1.00 \mathrm{E}+34 \mathrm{~cm}-2 \mathrm{sec}-1$ \\
\hline Initial lifetime ${ }^{\star \star}$ & 10.66 & 5.1 & 9.0 & 24 & 13 hours \\
\hline 15 hour integrated ${ }^{\star \star}$ & 3.75 & 88 & 306 & 45 & $377 \mathrm{pb}-1$ \\
\hline Initial lifetime ${ }^{\star \star \star}$ & 9.22 & 4.76 & 7.45 & & 11 hours \\
\hline 15 hour integrated ${ }^{\star \star \star}$ & 3.49 & 84 & 278 & & $354 \mathrm{pb}-1$ \\
\hline Total Cross Section & 75 & 86 & 94 & 94 & 94 mbarns \\
\hline
\end{tabular}

Options for the Tevatron Tunnel, December 23, 1993 Page 13 


\subsection{Antiproton Proton Using New Magnets}

This option utilizes the existing antiproton source and requires only one high energy magnet ring in the Tevatron tunnel. The magnets of this ring must have sufficient magnetic aperture to allow injection without loss of the separated proton-antiproton beams, and if fixed target physics is to be retained, slow extraction at $800 \mathrm{GeV}$. This option is based on replacing four Tevatron 4.4 Tesla dipoles with two 7.7 Tesla dipoles, and keeping the total bend angle the same. The dipole may be a follow up to a basic design provided earlier at Fermilab7. Variants on this option use 6.6 Tesla dipoles or 8.8 Tesla dipoles. Specific examples are based on a SSC collider dipole cross section or an SSC HEB dipole cross section.

\subsubsection{Lower Temperature Tevatron}

As an aside, it should be pointed out that the option of operating the existing Tevatron magnets at a lower temperature than the present $3.6 \mathrm{~K}$ is not viable. The Tevatron magnets, in principle, will reach $6.6 \mathrm{~T}$ at $1.8 \mathrm{~K}$. However, at this field, the stresses in the magnets will be a factor of 2.25 greater than the design value. This will lower the number of cycles to fatigue failure, degrade the field quality due to coil motion, and will require the removal of the magnets from the tunnel for training or intolerable in situ training in the tunnel. The higher fields will also increase by a factor of 1.5 the persistent current fields at injection. Finally, because of the large heat leak of the Tevatron magnet cryostat, approximately 20 Watts per $21 \mathrm{ft}$ dipole 8 without a ramp, the refrigerator output will need to be increased by at least a factor of 3 .

\subsubsection{Magnet Choices}

Possible dipole choices for a single new ring in the Tevatron tunnel are the SSC Collider dipole 9 , a single bore version of the LHC dipole, the 1989 design SSC HEB dipole, or a dipole which is very similar in design to the HEB dipole and is based on a Fermilab high

\footnotetext{
7 TM-1641 "The Design of a Large Aperture High Field Dipole", by Fady Harfoush et al., December 1989.

8 Tom Nicol, private communication.

9 "20 TeV Collider Dipole Design Possibilities", A. McInturff et al, Proceedings of The 12th International Conference on High-Energy Accelerators, pg 72.
} 
field design10. The SSC Collider dipole has a coil aperture of $5 \mathrm{~cm}$ and was designed to operate at $6.6 \mathrm{~T}$ and $4.2 \mathrm{~K}$. The original version of the LHC dipole has a $5 \mathrm{~cm}$ coil aperture and operates at $10 \mathrm{~T}$ and 1.8 $\mathrm{K}$. The original HEB dipole has a coil aperture of $7 \mathrm{~cm}$ and was designed to operate at $6.26 \mathrm{~T}$ and $4.2 \mathrm{~K}$. The Fermilab high field dipole has a coil aperture of $7 \mathrm{~cm}$ and was designed to operate at 6.6 $\mathrm{T}$ and $4.2 \mathrm{~K}$, or $8 \mathrm{~T}$ and $1.8 \mathrm{~K}$.

Tooling for the SSC Collider dipole exists and at least 12 dipoles have been assembled and successfully tested. However, its aperture is probably marginal for this application. If fields higher that $6.6 \mathrm{~T}$ are required, the magnet will need to be operated at a temperature colder than $4.2 \mathrm{~K}$ and the adequacy of its preload at higher fields will have to be confirmed.

The LHC dipole has the highest field that one can probably contemplate for a NbTi magnet with a $5 \mathrm{~cm}$ bore and high series production. Its coil dimension demonstrates the wide cable necessary to withstand the extremely high Lorentz forces present at this high field. This coil can be used as an example of present high field magnet technology and as a possible coil if a limited number of higher field than lattice dipoles are required near the low beta or injection/extraction straight sections. As with the SSC Collider dipole, its original $5 \mathrm{~cm}$ dipole probably has marginal aperture for our application. The bore field of this magnet has been recently lowered and its coil aperture has been increased to $5.6 \mathrm{~T}$.

Both the original HEB dipole and the Harrison dipole have the required field aperture due to their larger coil diameters. The Harrison dipole was designed specifically to satisfy the slow extraction and separate orbits presently in the Tevatron. A full sized magnet of neither design has been built and the tooling for these magnets is not available at this time. Because of their larger coil bore, these magnets are more expensive than the SSC Collider magnet to manufacture.

\section{0 .3 Specific Magnet Choice}

An interesting exercise, assuming that we are free to design a magnet specifically for a high energy proton-antiproton option for

10 TM-1641, ibid.

Options for the Tevatron Tunnel, December 23, 1993 Page 15 
the Tevatron tunnel, is to explore the magnetic field limit of a magnet operating at our present $3.6 \mathrm{~K}$ temperature and with sufficient aperture for slow extraction and separated orbits at injection. A $6 \mathrm{~cm}$ coil diameter, $7.7 \mathrm{~T}$ bore field with aluminum coil collars is suggested as a starting goal for such a magnet. This option gives a significant increase in top production over the expected yield after the main injector upgrade without the need for a $1.8 \mathrm{~K}$ refrigerator. If the coils are designed to tolerate the stresses present at $8.8 \mathrm{~T}$, a further increase in top production could be obtained after an eventual $1.8 \mathrm{~K}$ upgrade of the refrigerator. The peak field in the lattice dipoles may also be limited by the achievable low beta insertion gradients.

\subsection{Energy}

The field in the SSC based dipoles can be raised to 7.7 Tesla by incorporating two changes. Firstly, the addition of iron ${ }^{11}$ on the yoke would be needed to correct the sextupole field at high field.

Secondly, $3.5^{\circ} \mathrm{K}$ helium would be used instead of $4.5^{\circ} \mathrm{K}$ helium to increase the short sample limit. The presently installed Tevatron cryogenic system already provides $3.5^{\circ} \mathrm{K}$ helium. If this were done, the center of mass energy would be $3.5 \mathrm{TeV}$.

The lattice quadrupoles in the Tevatron require 76 Tesla / meter for $1 \mathrm{TeV}$ and are 1.676 meters (66 inches) long. A $1.75 \mathrm{TeV}$ direct replacement would require 133 Tesla / meter. The present 75 $\mathrm{mm}$ aperture low beta quads are designed to achieve 140 Tesla / meter at $4.5 \circ \mathrm{K}$. These considerations indicate that the lattice quads are not expected to limit the energy of the collider.

\subsection{Energy Limitations}

The devices other than the superconducting dipoles and lattice quadrupoles in the accelerator may limit the energy. The conventional devices do not depend on superconducting technology and thus do not benefit from the increase in magnetic field. The electrostatic separators are not expected to be a limit as shown in the section on "Crossing Angle" below. The RF is not a problem since the acceleration ramp can be slowed down. The present Tevatron low beta quadrupoles have a gradient of 140 Tesla / meter with a $75 \mathrm{~mm}$

11 James Strait, private communication. 
coil diameter aperture. New low beta quadrupoles would need to achieve a gradient of 245 Tesla / meter. Additional R\&D is needed to achieve this and it is expected that a $1.80 \mathrm{~K}$ cryogenic system will be needed for the low beta quads. The lambertsons for the proton abort at $\mathrm{CO}$ are not considered a collider energy limit since they can be removed from the lattice for collider operation. The lambertsons and kickers used for injection at FO are not a problem for $150 \mathrm{GeV}$ injection, as that is the present injection energy.

The probable limit to the collider energy is given by the AO internal abort. The kickers may be one of the limits to the collider energy if additional R\&D cannot demonstrate a system capable of $1.75 \mathrm{TeV}$ operation instead of $1 \mathrm{TeV}$. In addition, the present $\mathrm{AO}$ beam dump absorber is well matched to ground water limitations. Finally, the thickness of the beam absorber must be re-evaluated for the higher energy to assure that the superconducting magnets downstream of the beam absorber do not absorb enough energy to quench.

The limit on the fixed target extraction energy is expected to remain at $1 \mathrm{TeV}$ due to the electrostatic wire septa at $\mathrm{DO}$, the extraction lambertsons at $\mathrm{A} 0$, the kickers and lambertsons for the beam dump at $\mathrm{CO}$, the beam line devices and shielding in switchyard and the external beam lines.

The ratio of maximum beam energy to injection beam energy is of some concern for superconducting accelerators. For the Tevatron, this ratio is 6.7: $1 \mathrm{TeV}$ top energy and $150 \mathrm{GeV}$ injection. The Tevatron requires careful compensation of persistent currents to avoid beam emittance blow up at injection and at the start of the ramp. Another example from an operating accelerator is given by HERA at DESY where this ratio is $20: 800 \mathrm{GeV}$ top energy and $40 \mathrm{GeV}$ injection. HERA requires the use of real time feedback to control the field quality at injection and at the start of the ramp. For the RHIC design this ratio is $6.25: 250 \mathrm{GeV}$ top energy and $40 \mathrm{GeV}$ injection. For RHIC, however, persistent currents are expected to be less of a problem than in the Tevatron due to the choice of cable. The SSC HEB was redesigned to be $2 \mathrm{TeV}$ instead of $1 \mathrm{TeV}$ to allow this ratio to be only 10 for the SSC Main Ring: $20 \mathrm{TeV}$ top field compared to $2 \mathrm{TeV}$ injection. The HEB itself also has this ratio as 10: $2 \mathrm{TeV}$ top energy and $200 \mathrm{GeV}$ injection. This ratio for the $3.5 \mathrm{TeV}$ center of mass collider considered here is $11.7: 1.75 \mathrm{TeV}$ per beam using the 150 GeV Main Injector as an injector. This ratio is not expected to be a 
problem for the accelerator considered here if the cable is properly chosen. Calculations as well as measurements of field quality should be done to assure that this be the case.

\subsection{Crossing Angle}

For 21 bucket spacing, the beams will collide head on as they do now in the Tevatron. The present separator design is based on a maximum voltage of $120 \mathrm{KVolts}$ which provides five sigma separation for $1 \mathrm{TeV}$ beams with emittances of $30 \pi \times 10^{-6} \mathrm{~m}$. At present, they run at $80 \mathrm{KV}$ for $900 \mathrm{GeV}$. Since the beam size shrinks as the square root of the energy, the required separator voltage required to maintain the same separation only goes up as the square root of the energy. Thus, at $1.75 \mathrm{TeV}$ the separators need to run at:

$$
112 k V=80 k V \sqrt{1.75 / 0.9}
$$

If the separators can be successfully conditioned to run at the design voltage, this should not present an operational problem with sparking.

For 7 bucket spacing, the beams would collide with a crossing angle. The present location of the separators is such that the first satellite crossings encounter one another inside the separators. A set of separator voltages must be developed which provide sufficient separation, and a crossing angle at the interaction point is the price one must pay in luminosity. In addition, one must assure that the low $\beta$ magnets are designed to have an aperture large enough to handle the separated beams where $\beta$ is largest if necessary.

\subsection{Luminosity, Event Rates, and Bunch Spacing}

The luminosity is approximately 1.75 times that provided by the Main Injector due to the increase in energy from $1 \mathrm{TeV}$ to 1.75 $\mathrm{TeV}$ as shown by:

$$
L=3(p / m) \frac{N_{p} N_{\bar{p}} B}{\beta^{*}\left(\varepsilon_{p}+\varepsilon_{\bar{p}}\right)} f G\left(\beta^{\star}, \sigma_{I}\right)
$$


A decrease in the bunch length $(\sigma)$ leads to an increase in luminosity slightly larger than the factor of 1.75 . Table 1a shows that one obtains an integrated luminosity of $41.7 \mathrm{pb}^{-1}$ per week.

As shown in Tables 1a and 2a, an antiproton proton collider operating at $\mathrm{E}_{\mathrm{cm}}=3.5 \mathrm{TeV}$ can produce about $2.07 \times 10^{32} \mathrm{~cm}^{-2} \mathrm{sec}^{-}$ 1 , and provide $41.7 \mathrm{pb}^{-1}$ per week. It is expected ${ }^{12}$ to produce about:

420 top pairs per week assuming $\mathrm{m}_{\mathrm{top}}=200 \mathrm{GeV}$, and 3500 top pairs per week assuming $\mathrm{mtop}=140 \mathrm{GeV}$.

The bunch spacing for this collider is that provided with the Main Injector, and the number is interactions per crossing is expected to be 2.89 . The initial design was for 36 bunches with a minimum of 21 bucket spacing between them, or a $396 \mathrm{nsec}$ minimum time between crossings. However, it is also possible to provide 72 bunches with a minimum of 7 bucket spacing between them, or a $132 \mathrm{nsec}$ minimum time between crossings. The latter is preferred at the higher energies to reduce the number of interactions per crossing. It may also be possible to provide 108 buckets which would reduce the interactions per crossing to 1.93.

The present plans for the $\mathrm{CDF}$ and $\mathrm{D} 0$ detector upgrades will accommodate the $132 \mathrm{nsec}$ time between crossings.

\subsection{Luminosity Limitations}

Table 1 shows $25 \pi \times 10^{-6} \mathrm{~mm}$-mrad for the proton transverse emittance. This is required for the coalesced beam to limit the antiproton tune shift to 0.016 . However, for the uncoalesced beams, the emittance could be reduced to $20 \pi \times 10^{-6} \mathrm{~mm}$-mrad without encountering this limitation. In addition, bunched beam cooling is more effective with the low intensity bunches.

For the antiproton proton option, the luminosity is limited by the beam beam tune shift of the antiprotons caused by the proton intensity and emittance. Thus, reducing the proton emittance will

12 Derived from Attachment to this report "Physics Sensitivity of Fermilab Machine Options", December 1993 provided by Christopher T. Hill and Stephen J. Parke. 
not increase the luminosity due to this limit. The tune shift of the protons caused by the antiprotons not a limitation. Thus, one can increase the luminosity by decreasing the antiproton emittance. However, since the antiproton emittance is already smaller than the proton emittance, this will only have a limited effect on the luminosity.

For the antiproton proton collider, the limitation on the luminosity and the integrated luminosity is the antiproton production rate. If this rate could be doubled, the integrated luminosity could be doubled if this led to a doubling the number of antiprotons per bunch. The proton beam beam tune shift would still small as shown in the Table. The total number of antiprotons required from the stack in these options is $130 \mathrm{mAmps}$ divided by the efficiency of the transfer from the source to low beta. If this efficiency is $70 \%$, then $186 \mathrm{mAmps}$ would be used from the stack. If 15 hours are allowed to replenish the stack and stacking uptime is $70 \%$, then the stacking rate must be $18 \mathrm{mAmps} /$ hour to replenish the stack.

\subsection{Dipole Sagitta}

The sagitta for a dipole is given by

$$
s=R[1-\cos (\theta / 2)]
$$

where $R$ is the bend radius and $\theta$ is the bend angle. For small bend angles the sagitta is given approximately by

$$
s=R \theta^{2} / 8
$$

Using $\theta=L / R$, where $L=$ the length of the magnet, the sagitta may be written as either

$$
s=L \theta / 8
$$

or

$$
s=L^{2} /(8 R) .
$$


For the Tevatron, $\mathrm{L}=6.12$ meters and $\theta=8.12 \mathrm{mrad}$ yields $\mathrm{s}=6.21$ $\mathrm{mm}$ for the Tevatron dipole sagitta.

Since the bend radius for SSC dipoles is postulated to be the same as the Tevatron dipoles, the sagitta scales with the square of the magnet length, that is:

$$
s_{s s C}=s_{\text {Tevatron }}\left(L_{s s C} / L_{\text {Tevatron }}\right)^{2} .
$$

For the SSC dipoles, $\mathrm{L}=12.5$ meters, and $\mathrm{s}=25.9 \mathrm{~mm}$.

The aperture is $50 \mathrm{~mm}$ for the SSC dipoles, and thus, if the sagitta were not taken into the account, it would use up half the aperture. It is not expected 13 to be difficult to make the magnets with the proper sagitta, and this will be assumed to be true.

\subsection{Magnet Aperture}

A proton-antiproton collider operating at center-of-mass energies greater than $2 \mathrm{TeV}$ in the Fermilab tunnel requires a single ring of new dipoles capable of operating at fields higher than $4.4 \mathrm{~T}$. The Tevatron magnets utilize a magnetic horizontal aperture of +- 20 $\mathrm{mm}$ for slow extraction in fixed target operation and separated beam injection during collider operation. Part of this aperture is required for beam steering errors. A physical and magnetic aperture proposal less than this value requires convincing calculations to be taken seriously.

In the following, the injection aperture is estimated from a general and specific point of view. The aperture required during collisions is also considered.

Another important requirement on magnet aperture is not considered here. It is the requirement that the aperture be large enough to accommodate errors (injection, closed orbit, etc.) so that the machine is operable. Thus, the apertures derived below are considered minimum apertures, rather than comfortably large apertures.

13 James Strait, private communication.

Options for the Tevatron Tunnel, December 23, 1993 Page 21 


\subsubsection{Injection Aperture From a General Point of View}

Transverse beam width is determined by the invariant transverse emittance and, through the mechanism of dispersion, energy spread. Representative sizes at a particular location in the ring are set by the simplest, first order approximation for the transverse displacement of a proton on the $\mathbf{n}_{\mathrm{th}}$ turn from its ideal position,

$$
x_{n}=D \frac{\Delta p}{p}+\sqrt{\frac{\beta \varepsilon}{\pi}} \sin (2 \pi n v+\phi),
$$

where $\varepsilon$ is the "emittance of the orbit," $\Delta p / p$ the fractional change in longitudinal momentum from an ideal value, and $D$ and $\beta$ are respectively the dispersion and envelope function of the lattice at the location of interest. ( $\phi$ is an arbitrary phase chosen to fit the initial condition.) Although details may change, the scales of the lattice functions are set by the tune and the geometry of the tunnel, according to

$$
\begin{aligned}
& \beta \sim\left\langle\frac{1}{\beta}\right\rangle^{-1} \sim \frac{R}{v} \sim 10^{3} m / 20 \sim 50 m \\
& D \sim \frac{\beta}{v} \sim 2.5 m
\end{aligned}
$$

Using $\beta \sim 50 \mathrm{~m}$ is a little too generous when compared to the horizontal $\beta$ in the existing Tevatron at injection. For most of the ring, $\beta$ fluctuates between $50 \mathrm{~m}$ and $150 \mathrm{~m} . \beta \sim 150 \mathrm{~m}$ will be used as the limit in the dipoles for this estimate. Also, a dispersion of $D \sim 2.5$ $\mathrm{m}$ is too generous, and the more conservative value of $6 \mathrm{~m}$ will be used.

In the expression for $x_{n}, \varepsilon$ is the emittance referred to one standard deviation of the beam width. The values listed in Table $1 \mathrm{a}$ and $1 \mathrm{~b}$ are invariant emittances referred to $95 \%$ of the beam. To adjust them, divide by 6 to change $95 \%$ to $1 \sigma$, and divide by the relativistic factor 


$$
\beta_{\mathrm{o}} \gamma_{\mathrm{o}}=\mathrm{pc} / \mathrm{mc}^{2}=150 / 0.938 \sim 160
$$

to change from "invariant" to actual emittance. Thus,

$$
\sqrt{\frac{\beta \varepsilon}{\pi}} \sim\left(\frac{1}{6 \beta_{o} \gamma_{o}} \frac{\beta \varepsilon_{i n v}}{\pi}\right)^{1 / 2} \sim\left\{\begin{array}{cc}
1.3 m m, \varepsilon_{i n v} / \pi= & 10 \mathrm{~mm}-\mathrm{mrad} \\
1.5 & 15 \\
1.8 & 20 \\
2.0 & 25
\end{array}\right\}
$$

To estimate $\Delta p / p$, we will use the expression

$$
\Delta E=\sqrt{\frac{2 V E}{\pi|\eta| h}}
$$

for the energy spread in the beam. Here $\mathrm{V}$ is the RF voltage, $\mathrm{E}$ is the energy, $\eta$ is the momentum compaction, and $h$ is the number of buckets. At $150 \mathrm{GeV}$ injection energy ${ }^{14}$, protons are captured in an $800 \mathrm{kV}$ bucket and antiprotons in a $530 \mathrm{kV}$ bucket. Assuming a bucket is filled and using $\gamma_{t}=18.85,|\eta| \sim 1 / \gamma_{t}{ }^{2}$ and $h=1113$ provide the result,

$$
(\Delta E) \sim\left\{\begin{array}{ll}
0.16 \mathrm{GeV}, & p \\
0.13 \mathrm{GeV}, & \bar{p}
\end{array}\right\}
$$

At these energies, $\Delta p / p \sim \Delta E / E$. . The rms energy spread is given approximately by $\sigma_{E}=(\Delta \mathrm{E})_{\max } / 2$. The contribution to the beam width is

$$
D \sigma_{p} / p \sim D \sigma_{E} / E \sim\left\{\begin{array}{ll}
3.2 \mathrm{~mm}, & p \\
2.6 \mathrm{~mm}, & \bar{p}
\end{array}\right\}
$$

We now add the numbers in quadrature, choosing the appropriate values for the invariant emittance from Table 1 .

$$
1 \sigma \sim\left\{\begin{array}{ll}
\sqrt{2.0^{2}+3.2^{2}}=3.8 \mathrm{~mm}, & p \\
\sqrt{1.5^{2}+2.6^{2}}=3.0 \mathrm{~mm}, & \bar{p}
\end{array}\right\}
$$

14 Ioanis Kourbanis, private communication.

Options for the Tevatron Tunnel, December 23, 1993 Page 23 
For stability against the long range beam-beam interaction the proton and antiproton orbits are separated by at least three times the proton standard deviation. Adding five standard deviations in beam width to either side of this provides the minimum diameter of the "good-field" region.

$$
d=3 \times 3.8+5(3.8+3.0)=45 \mathrm{~mm}=\left\{\begin{array}{lll}
60 \% & \text { of } & 7.5 \mathrm{~cm} \\
80 \% & \text { of } & 5.6 \mathrm{~cm} \\
90 \% & \text { of } & 5.0 \mathrm{~cm}
\end{array}\right\}
$$

If we adopt as rule-of-thumb that the "good-field-diameter" of a superconducting magnet is about $50-60 \%$ of the physical aperture, the $7.5 \mathrm{~cm}$ Tevatron magnet barely satisfies this criterion. On the other hand, both the $5.6 \mathrm{~cm}$ LHC magnet and the $5 \mathrm{~cm}$ SSC magnet are uncomfortably small and would require detailed and believable analysis to justify using.

\subsubsection{Iniection Aperture From a Specific Point of View}

The aperture required for injection onto helical orbits is considered here. For the case appropriate to $150 \mathrm{GeV}$ injection into a lattice like the Tevatron, in which $\beta=100$ meters and $\varepsilon=30 \pi \times 10^{-6}$ $\mathrm{mm}$-mrad, $\sigma=1.77 \mathrm{~mm}$ for the contribution from the transverse emittance. The beam is considerably larger than this at the high dispersion locations. For $\sigma_{p}=0.5 \times 10^{-3}$ and a dispersion of 6 meters, $\sigma=3 \mathrm{~mm}$ for the contribution from the longitudinal emittance. Combining these two in quadrature gives an rms beam size at the high dispersion locations of $\sigma=3.45 \mathrm{~mm}$. The aperture required to separate two such beams by 3 sigma and contain +-5 sigma of the beams is $13 \sigma=45 \mathrm{~mm}$.

The dynamic aperture 15 of the Tevatron is $50 \mathrm{~mm}$ compared to its coil diameter of $75 \mathrm{~mm}$. This is barely adequate to accommodate injection onto helical orbits which requires $45 \mathrm{~mm}$. If the dynamic aperture were to scale with the coil radius, then the dynamic aperture of a $50 \mathrm{~mm}$ aperture dipole would be about $50 \mathrm{~mm} \mathrm{x}$ $(50 / 75)=33 \mathrm{~mm}$. This is too small to contain the $45 \mathrm{~mm}$ required

15 Measurements by Bruce Hanna and David Finley. 
for injection onto helical orbits. On the other hand, the dipole discussed earlier ${ }^{16}$ is adequate to contain these helical orbits.

\subsubsection{Aperture During Collisions}

The aperture required at high field for operation of helical orbits during a store is considered here.

For the case appropriate to $1.75 \mathrm{TeV}$ beam, in a lattice like the Tevatron, in which $\beta=100$ meters and $\varepsilon=30 \pi \times 10^{-6} \mathrm{~mm}-\mathrm{mrad}, \sigma=$ $0.52 \mathrm{~mm}$ for the contribution from the transverse emittance. The beam is considerably larger than this at the high dispersion locations. For $\sigma_{\mathrm{p}}=0.15 \times 10^{-3}$ and a dispersion of 6 meters, $\sigma=0.9 \mathrm{~mm}$ for the contribution from the longitudinal emittance. Combining these two in quadrature gives an rms beam size at the high dispersion locations of $\sigma=1 \mathrm{~mm}$. The aperture required to separate two such beams by 3 sigma and contain +-5 sigma of the beams is $13 \sigma=13 \mathrm{~mm}$. If one wanted to contain +-10 sigma and maintain a separation of 5 sigma, one would need an aperture of $25 \sigma=25 \mathrm{~mm}$.

The dynamic aperture required of the triplet low beta quadrupoles at injection may be smaller than that required of the dipoles and quadrupoles in the arcs. This can be done if a separator solution can be found which allows the beams to pass through the center of these quadrupoles during injection 17. Assuming dispersion is zero, and that $\beta=200$ meters in the triplet at injection, one obtains $\sigma=2.5 \mathrm{~mm}$ in the triplet for the $150 \mathrm{GeV}$ beams. The aperture required to contain $+-5 \sigma$ at injection is $25 \mathrm{~mm}$. During collisions of the $1.75 \mathrm{TeV}$ beams, if one assumes $\beta=1000$ meters and the dispersion is zero, one obtains $\sigma=1.64 \mathrm{~mm}$. In this case, the aperture in the low beta quads required to contain $+-5 \sigma$ during collisions would be $16 \mathrm{~mm}$.

However, if a crossing angle is required, the aperture in the low beta quads needs to be larger.

16 TM-1641, ibid.

17 This, of course, defeats the advantage of having the beams separated everywhere at injection as is done now. Whether this can actually be done with the cogging required to inject 36 or more bunches has not be carefully thought through. 


\subsection{Resonant Extraction}

An additional constraint on the aperture is provided by the requirement for resonant extraction at high field. At this time, it has not been demonstrated whether the $5 \mathrm{~cm}$ aperture SSC magnets can or can not support resonant extraction, since this was not required of the SSC project. However, resonant extraction from a ring composed of the new magnets can probably be done. The worst case is posed by the $5 \mathrm{~cm}$ aperture dipoles. The Tevatron dipoles have a coil aperture of $75 \mathrm{~mm}$ and require an aperture of $40 \mathrm{~mm}$ for resonant extraction. If one assumes the available aperture scales with the coil aperture, one has $27 \mathrm{~mm}$ for the available aperture in the dipoles with a $50 \mathrm{~mm}$ coil aperture. In order to confine the extracting beam to this aperture in the arcs some adjustments must be made. These include tripling the strength of octupoles used to drive extraction. In addition, the distance between the extraction septum wires must be reduced by a factor of 0.7 , and the half integer stop band must be increased by a factor of 1.45 .

A simplified analysis supporting the above contention is provided here ${ }^{18}$. Starting with a model Hamiltonian ${ }^{19}$ one can analyze resonant extraction in terms of simple scaling relations, which constrain a basic set of four quantities describing resonant extraction process. These four quantities are:

- $\mathrm{a}^{\max }$ maximum betatron amplitude of the circulating beam

- $x^{\text {sep }}$ septum offset

- $\varepsilon^{\text {ext }} \quad$ extracted beam emittance

- $f$ extraction inefficiency; the ratio of the number of particles hitting a septum wire to the number of extracted particles

The above listed quantities are in turn functions of the following three extraction parameters:

18 Alex Bogacz provided this analysis in consultation with John Johnstone.

19 John A. Johnstone, A Simplified Analysis of Resonant Extraction at the Main Injector, FERMI-Pub. September 1993. 
- $\delta$ fractional tune separation

half-integer resonance driven by harmonic

quadrupoles distributed on the $\mathrm{N}$-th harmonics, so that

$\frac{N}{2}$ is the closest half integer to the horizontal tune, $v$

$$
\delta=\frac{N}{2}-v
$$

- $\chi \quad$ phase-space orientation at the septum half-integer stop-band driven by two families of orthogonal quadrupoles of strengths $\mathrm{q}_{\mathrm{s}}$ and $\mathrm{q}_{\mathrm{c}}$ The phase space orientation at the septum is defined by $\chi$

$$
\chi=\arctan \left(\frac{q_{s}}{q_{c}}\right)
$$

- $\lambda \quad$ octupole strength

0-th harmonic octupoles, which guarantees unstable configuration of the phase space

$$
\lambda=\frac{1}{2 \pi} \int_{L} d s \frac{B^{\prime \prime \prime} \beta^{2}}{96 B_{o} \rho}
$$

Following model Hamiltonian analysis ${ }^{20}$ one obtains the following set of scaling relationship for our basic set of extraction characteristics

$$
\begin{aligned}
& a^{\max }=\left(\frac{\delta \beta^{\max }}{3 \lambda}\right)^{1 / 2} \cdots[\chi] \\
& x^{\operatorname{sep}}=\left(\frac{\delta \beta^{\operatorname{sep}}}{6 \lambda}\right)^{1 / 2} \cdots[\chi] \\
& \varepsilon^{e x t}=\frac{\delta^{3}}{6 \lambda} \cdots[\chi]
\end{aligned}
$$

20 John A. Johnstone, ibid.

Options for the Tevatron Tunnel, December 23, 1993 Page 27 


$$
f=\left(\frac{6 \lambda}{\delta \beta^{s e p}}\right)^{1 / 2} \frac{w}{\delta} \ldots[\chi]
$$

Here, ... $[\chi]$ denotes generic dependencies of the above quantities of the phase-space orientation at the septum, $w$ is the width of the septum wire.

The Tevatron dipoles have a $75 \mathrm{~mm}$ coil aperture and the horizontal dipole aperture in the $\operatorname{arcs}^{21}$ is $a_{0} \max =+-20 \mathrm{~mm}$. For new magnets, such as the HEB $50 \mathrm{~mm}$ magnets, one can scale down the corresponding horizontal dipole aperture at the arc, a $\max$ (assuming the same lattice) by

$$
\mathrm{a}^{\max }=\rho \mathrm{a}_{0}^{\max }, \rho<1
$$

Imposing the same inefficiency for the resonant extraction

$$
f=f_{0}
$$

and applying the scaling relationships given by Eqs.(1.8.4)-(1.8.7), one obtains the following scaling for the extraction parameters:

$\begin{array}{cccccc}\frac{\mathrm{a}^{\max }}{\mathrm{a}_{0} \max } & \frac{\mathrm{x}^{\mathrm{sep}}}{\mathrm{x}_{\mathrm{O}}^{\mathrm{sep}}} & \frac{\mathrm{f}}{\mathrm{f}_{\mathrm{O}}} & \frac{\delta}{\delta_{o}} & \frac{\varepsilon^{\text {ext }}}{\varepsilon_{o}^{\text {ext }}} & \frac{\lambda}{\lambda_{o}} \\ \rho & \rho & 1 & \rho^{-1} & 1 & \rho^{-3}\end{array}$

This table shows that if one wants to have an extraction efficiency from a ring composed of the smaller aperture dipoles to be the same as the Tevatron extraction efficiency, then one requires a smaller septum offset, larger tune separation and stronger octupoles. The extracted beam emittance stays the same.

For the $50 \mathrm{~mm}$ dipoles compared to the $75 \mathrm{~mm}$ Tevatron dipoles, $\rho=a^{\max } / a_{0} \max =50 / 75=0.67$. In this case,

21 H.T. Edwards and M. Harrison, Good Field Region of the Design Bend Magnet and Expected Behavior of Extraction, FERMI-UPC No. 66, October 1978. 


\begin{tabular}{llllll}
$\frac{\mathrm{a}^{\max }}{\mathrm{a}_{0}{ }^{\max }} \quad \frac{\mathrm{x}^{\mathrm{sep}}}{\mathrm{x}_{\mathrm{O}}^{\mathrm{sep}}}$ & $\frac{\mathrm{f}}{\mathrm{f}_{\mathrm{O}}}$ & $\frac{\delta}{\delta_{o}}$ & $\frac{\varepsilon^{e x t}}{\varepsilon_{o}^{e x t}}$ & $\frac{\lambda}{\lambda_{o}}$ \\
\hline 0.67 & 0.67 & 1 & 1.5 & 1 & 3.4
\end{tabular}

Whether one can accept an increase of a factor of 3.4 in octupole strength needs further study. It is limited by higher harmonics of the octupole (2-nd, 4-th), which may drive half- and quarter-integer resonances. The second resonance is especially dangerous in terms of its distorting effect on the phase-space configuration and this consideration sets the limit on the octupole strength. Assuming one can accept the increased value of the octupole, the horizontal dipole aperture for a $50 \mathrm{~mm}$ would be: $\mathrm{a}^{\max }=0.69 \mathrm{a}_{0} \max _{=}+-13.3 \mathrm{~mm}$, or $26.6 \mathrm{~mm}$ full width.

\subsection{Fixed Target Ramp Rate}

This section considers the requirements on superconducting magnets for ramping for a fixed target physics program.

\subsubsection{Tevatron Ramp Rate}

If the Tevatron is replaced by a new accelerator, it would be good if the new accelerator could maintain (or improve upon) the Tevatron's for fixed target cycle rate. There are four factors that limit the ramp rate of the Tevatron:

- maximum voltage available from the magnet power supplies

- maximum voltage available from the RF cavities: this limits the energy gain per turn. The magnetic field must follow the proton energy.

- magnet di/dt limit: higher di/dt more heat dissipated in the cable

- magnet overall life: mechanical fatigue depends on the total number of cycles. Increasing the ramp rate will result in a shorter cycle time. 
In fixed target mode, the objective from an operational standpoint is to maximize the integrated number of protons delivered to the experiments. Since the rate at which protons can be delivered is not always limited by the experiments, there are two possible strategies:

(1) increase the number of protons and spill them over a longer period of time and

(2) reduce the dead time by ramping the magnets faster. In the Tevatron, the 60 second fixed-target cycle is divided approximately equally between up ramp, flat-top and down ramp.

Assuming that the number of protons stored in the accelerator is fixed by other considerations, more protons could be delivered over a given period of time by increasing the ramp rate and reducing the cycle time. It appears reasonable to conclude that the ramp rate was simply set to the highest practical value.

Although there is a limit to the power and the voltage that can be delivered by the magnet power supplies, this was not the determinant factor in setting the ramp rate for the Tevatron fixed target cycle. The same comment applies to the number of RF cavities and the RF power supplies. The main consideration was the heat load associated with cyclic losses in the cable22. Although there is experimental evidence that the Tevatron magnets could be ramped up 1.5 to 2 times as fast as they are presently 23 the probability of a quench under such conditions is not acceptable. Another important consideration is mechanical fatigue. Although no Tevatron magnet has failed due to mechanical fatigue yet, increasing the ramp rate reduces the life expectancy of the magnets.

\subsubsection{Cyclic and Time-Dependent Effects in Superconducting Magnets}

The cyclic nature of the field in superconducting accelerator magnets results in a heat load, called the cyclic heat load. Since cryostats are usually designed to minimize the (static) heat leak, the cyclic heat load determines to a large extent the required refrigerator capacity of a fast cycling accelerator. By raising the

22 Karl Koepke, private communication.

23 Norman Gelfand, private communication. 
operating temperature of the magnets, the cyclic heat load reduces the critical current density and therefore limits the peak field of the magnets.

Hysteresis and eddy current heating are present both in the iron yoke and superconducting cable. The heat load from the aluminum collar and stainless-steel bore tube are due to eddy currents only. The losses due to inelastic mechanical motion in the flexing coil and collars are very small and can be ignored.

The energy loss per cycle per unit volume of cable can be estimated using the following approximate relation

$$
E=c_{1} \frac{1}{1+k} d J_{c} \Delta B+c_{2} \Delta B d B / d t
$$

where $c_{1}$ and $c_{2}$ are constants that depend on a particular magnet and $\mathrm{k}$ is the $\mathrm{Cu}: \mathrm{SC}$ ratio of the cable. $\mathrm{J}_{\mathrm{c}}$ and $\mathrm{d}$ are respectively the average critical current density and the filament diameter of the cable and $B$ is the span of the magnetic field variation.

\subsubsection{Comparison of Tevatron and HEB Dipoles}

A comparison of some of the magnet parameters for the Tevatron and HEB dipoles are given in the following table. 


$\begin{array}{llll} & & \text { Tevatron } & \text { HEB/SSC } \\ \text { Peak Field } & {[\mathrm{T}]} & 4.4 & 6.4 \\ \text { Peak Current } & {[\mathrm{A}]} & 4400 & 6600 \\ \text { Transfer Constant } & {[\mathrm{T} / \mathrm{kA}]} & 1.0 & 0.97 \\ \text { Cycle } & {[\mathrm{s}]} & & \\ \text { Inject } & & 0 & 10 \\ \text { Up Ramp } & & 20 & 50 \\ \text { Flattop } & & 20 & 10 \\ \text { Down Ramp } & & 20 & 50 \\ \text { dB/dt } & {[\mathrm{T} / \mathrm{s}]} & 0.2 & 0.1152 \\ \text { dI/dt } & {[\mathrm{A} / \mathrm{s}]} & 200 & - \\ \text { Period } & {[\mathrm{kA} / \mathrm{s}]} & 0.22 & 0.1250 \\ \text { Magnet Length } & {[\mathrm{m}]} & 6.0 & 15.0 \\ \text { Aperture } & {[\mathrm{mm}]} & 7.62 & 5.0 \\ \text { Inductance } & {[\mathrm{mH} / \mathrm{m}]} & 7.5 & 5.0 \\ \text { Filament size } & {[\mathrm{micron}]} & 8.0 & 6.0(2.5 ?) \\ \text { CurSC } & & - & 1.3-1.5(\mathrm{I} \text {.C.) } \\ & & & 1.8(\mathrm{O} . \mathrm{C.} .)\end{array}$

A comparison of the heat load of the Tevatron and HEB magnets are given in the following table.

Heat Load

Yoke Hysteresis

Yoke eddy

Collar eddy

Coil hysteresis

Coil eddy

Bore tube eddy

Total cyclic

Static

Margin

Total Losses
[W/magnet]

[W/magnet]

[W/magnet]

[W/magnet]

[W/magnet $]$

[W/magnet]

[W/magnet]

[W/magnet $]$

[W/magnet]

[W/magnet $]$
Tevatron HEB/SSC

$\begin{array}{ll}- & 1.0 \\ - & - \\ - & 0.2 \\ 5.9 & 3.6 \\ 0.93 & 2.1 \\ - & - \\ 6.83 & 6.9 \\ 7 \text { to } 10 & 0.8 \\ 1.3 & - \\ \sim 15 & \sim 7\end{array}$

If one wants to duplicate the performance of the Tevatron in fixed target mode with HEB style magnets, the HEB magnets have to run at roughly twice the ramp rate given in the above table. Using 
the superscripts 0 and 1 to distinguish design and scaled values, the cyclic losses are expected to scale roughly as:

eddy losses: $\quad\left[\frac{(d B / d t)^{(1)}}{(d B / d t)^{(0)}}\right]^{2}\left[\frac{\Delta B^{(1)}}{\Delta B^{(0)}}\right]$

hysteresis: $\left[\frac{\Delta B^{(1)}}{\Delta B^{(0)}}\right]$

In the HEB/SSC magnet, the cyclic losses are dominated by hysteresis losses in the SC cable. If the HEB ramp rate were doubled $(200 \mathrm{~A} / \mathrm{s}$ vs $100 \mathrm{~A} / \mathrm{s})$, these losses would increase by roughly $40 \%$. The ability of a magnet to sustain higher ramp rate is determined to a large extent by the effectiveness with which the heat can be removed from the superconductor. This depends, among other things on the geometry of the cooling channels, the helium flow rate, the type of insulation, the filament size (small filament = larger surface to volume ratio) and the superconducting material to copper ratio in the cable.

\subsubsection{Ramping the HEB Dipoles}

In the early stages of the Supercollider design, it was assumed that the HEB dipole magnets would be distinct from the collider ring dipoles. For economic reasons, it was later decided to use the collider magnets in the HEB ring. Early in the test program for SSC dipoles, it was realized that these magnets exhibit rather significant dependence of quench current on ramp rate. This dependence is of no serious consequence for the collider which was designed to ramp at $4 \mathrm{~A} / \mathrm{s}$. However, concerns have been expressed about the ability of the magnets to ramp at $100 \mathrm{~A} / \mathrm{s}$ as required for the HEB cycle. Published experimental data 24 indicates that at ramp rate of the order of $200 \mathrm{~A} / \mathrm{s}$, the quench current was below $4 \mathrm{kA}$ for some fraction of the magnets. While the exact cause of the quench current dependence on the ramp rate for the collider magnets needs to be identified, the data shows clearly that it is possible to ramp the SSC dipole magnets to $4.4 \mathrm{~T}$ at $200 \mathrm{~A} / \mathrm{s}$. There is little doubt that it

$24 \mathrm{~J}$. Kuzminski et al. "Quench Performance of 50-mm Aperture, 15-m-long SSC Dipole Magnets Built at Fermilab", Proceedings of the XVth International Conference on High Energy Accelerators, Hamburg Germany 1992, p 588. 
should be possible to build SSC style magnets that perform reliably at $200 \mathrm{~A} / \mathrm{s}$ by using a different cable with a smaller filament size (to reduce hysteresis losses) and by electrically insulating the strands of the cable to suppress eddy-currents. For the collider magnets, it was decided to save money by not insulating the strands of the cable because of the very slow ramp rate 25 .

The HEB magnets can probably be designed to ramp adequately for fixed target based on the following recent information from SSCL 26 . One 1.8 meter HEB model magnet with all-ebanol cable had been measured and found to have no measurable eddy current harmonics up to $64 \mathrm{Amps} / \mathrm{sec}$. They could not make harmonics measurements at higher ramp rates with their system. Therefore it is not ruled out (based on eddy current harmonics) that an HEB dipole could be used for a Tevatron replacement. However it is yet to be proven 1) that the harmonics are also negligible at $200 \mathrm{~A} / \mathrm{s}$ and 2 ) that the favorable results from this one short magnet are reproducible.

\subsection{Cryostat}

The cryostats of the magnets proposed in this report have not been optimized for the Tevatron tunnel or the Fermilab refrigerator. It is assumed that the cryostats will be redesigned to make them compatible with the Tevatron refrigerator and to minimize the vertical move of the detectors. This should be achievable without seriously degrading the heat leak specifications of the original cryostats.

The vacuum vessel for the SSC HEB magnets has an outer diameter of 0.6 meters and the beam pipe is approximately centered in the cryostat. The SSC Main Ring magnets have an outer diameter of 0.6 meters and the beam pipe is off center in the cryostat. These magnets will sit on the floor of the Tevatron tunnel, as shown in Figure $1 \mathrm{a}$ and $1 \mathrm{~b}$. The beam line for the HEB based collider will be 6 inches above that of the Tevatron, and the beam line for the collider based on the SSC Main Ring magnets will be 12 inches above the Tevatron. These changes require moving the detectors in the CDF

25 SSC Conceptual Design, March 1986, p 279.

26 This information is provided by Jim Strait in consultation with Arnaud Devred of SSC. 
and DO collision halls up by an equal amount. The removal of the Main Ring from the ceiling at CDF is expected to provide space to do this at $\mathrm{CDF}$.

The outer vacuum vessel also requires moving the Main Injector beam transfer line in F-sector up by a similar amount as shown in Figures 1c and 1d. This beam transfer line is used to provide protons to the antiproton production target, receive antiprotons from the source, as well as provide protons to the switchyard for the fixed target physics program. This move may require redesigning the beam transfer line to preserve the vertical dispersion match.

The outer vacuum vessel also requires modifications to the beam transfer lines between the Main Injector and the $3.5 \mathrm{TeV}$ collider since it is not at the same elevation as the Tevatron.

\subsection{Cryogenics Requirements}

The present Fermilab cryogenic system provides for the Tevatron. The HEB/SSC dipole cryogenic heat load is substantially less than for a Tevatron dipole. This is mostly the result of a different cryostat design. The Tevatron dipole has a room temperature iron yoke. The coil support structure is complicated and it has to withstand the forces between the yoke and the coil in case of asymmetry. Because the support structure provides a contact between the cold coil structure and the warm yoke, the static heat leak is rather high, i.e. of the order of $10 \mathrm{~W} /$ magnet. The SSC dipole yoke static heat leak is much smaller because the yoke is inside the cryostat. Assuming that the magnets are operating at the same temperature, the Tevatron refrigerators would easily have sufficient capacity for a ring of HEB/SSC magnets in the existing Tevatron tunnel.

One must keep in mind that the thermodynamic efficiency of the refrigerators decreases dramatically as the temperature is reduced. In practice, it takes approximately 700 Watts at room temperature to remove 1 Watt at $4.2 \mathrm{~K}$. This figure increases very rapidly when the temperature is decreased, at least as fast as 1/T (Carnot Cycle). 
The power leads in the Tevatron use much of the presently installed capacity, and an $R \& D$ program for power leads could alleviate this in a new accelerator.

\subsection{Installation and Commissioning}

The installation and commissioning described here assumes that nearly all the equipment for the $3.5 \mathrm{TeV}$ collider is available when the Main Injector installation begins. It is not necessary, but it is apparent that Fermilab's physics output will be maximized if the installation and commissioning of the Main Injector and this new accelerator are coordinated in the manner described here. If the equipment for the $3.5 \mathrm{TeV}$ collider is not available, the Main Injector would extract into the Tevatron after its commissioning. But the window of opportunity for overlapping the two major down times would have passed.

The installation of the Main Injector requires several changes to the Main Ring components in the Tevatron tunnel. These include the removal of several Main Ring quadrupoles and dipoles throughout the tunnel, and the reconfiguration of the FO and AO straight sections. Reconfiguration of the FO straight section includes the beam transfer lines from the Main Injector to the collider. The installation of the $3.5 \mathrm{TeV}$ collider described here requires additional modifications in F-sector as indicated in the section "Cryostat" above. In addition, the installation of the $3.5 \mathrm{TeV}$ collider requires the removal of the Tevatron, and it would probably be convenient to remove the remainder of the Main Ring as well except in F-sector.

Access to a certain section of the $3.5 \mathrm{TeV}$ collider would be prohibited when passing the Main Injector beam from FO to the switchyard. The prohibited section includes all of F-sector and parts of A-sector and E-sector. Installation of the parts of the $3.5 \mathrm{TeV}$ collider in these sections could be given priority in order to be out of the tunnel before the Main Injector needs it for beam. If properly staged and coordinated, interferences between the Main Injector and the 3.5 TeV collider requirements in this section of the Tevatron tunnel can be avoided. The remainder of the Tevatron tunnel (estimated to be about $3 / 4$ of the tunnel) is available for access when the Main Injector is extracting beam to the switchyard. The available section includes the CDF and DO collision halls, so the installation of the detectors can proceed as well. 
Besides extracting beam to switchyard, the Main Injector could commission other aspects of its operation required for collider operation before the $3.5 \mathrm{TeV}$ collider is available for beam. These include antiproton production, antiproton transfers from the source to the Main Injector, and coalescing of both protons and antiprotons. During this time the Main Injector can still be running a $120 \mathrm{GeV}$ fixed target program.

After the 3.5 TeV collider ring is installed, the cryogenics and power systems can be commissioned and made operational. The power systems include the main bus, the low beta inserts, the correction elements, and the beam transfer devices. Once the 3.5 $\mathrm{TeV}$ collider is ready for beam, one can continue to run the Main Injector fixed target program at a slightly reduced rate. This segue into simultaneous Main Injector fixed target running and collider running clearly maximizes the use of the new Fermilab accelerators.

\subsection{Elements of Cost}

\subsubsection{Summary of Cost Elements}

The proton antiproton option is expected to be the least expensive of the options presented in this paper, and poses the smallest technical risk as a project. It produces about 10 times as many tops per week as the Main Injector.

The elements of the cost can be divided into four parts: $R \& D$, production, installation, and operation.

R\&D on superconducting devices for $3.5 \% \mathrm{~K}$ :

7.7 Tesla dipoles, lattice and straight section quads

R\&D on superconducting devices for $1.8 \mathrm{OK}$ : low beta quads

$R \& D$ on conventional devices: beam dump kickers and absorber separators

Development of lattice design and engineering layout production of 38412.5 meter lattice dipoles production of 6 "half dipoles" as necessary for the 48 locations. production of about 200 lattice quads of two types production of straight section quads of three types 
production of about 200 correction packages of many types production of low beta quads

production of kickers and dump for beam dump if necessary installation of superconducting ring and connection to cryogenics operation of 3.5 degree cryogenics for Tevatron plus another accelerator

\subsubsection{Details of Cost Estimate for Dipoles}

The basis of the cost estimates for all dipoles in this report is given in this section.

Approximately 400 dipoles, each with a magnetic length of $12.2 \mathrm{~m}$, are required per ring in the Tevatron tunnel. The dipole will be similar in design and assembly to the SSC Collider magnet for which actual fabrication costs are available. The cost of magnets with other coil apertures and operating fields can be estimated as follows: The SSC Collider dipole is assumed to have been designed to the current limit of the superconducting cable, to the stress limit of the cable and insulation, and to the saturation limit of the iron yoke. Under these assumptions, the material costs of a similar magnet with unchanged bore field and different coil aperture can be estimated with a linear scaling of the apertures. The cost change due to a change in operating field from $6.6 \mathrm{~T}$ to $8.8 \mathrm{~T}$ while keeping the coil aperture constant can be obtained by scaling the cost linearly with field if the increased field is obtained by reducing the operating temperature from $4.2 \mathrm{~K}$ to $1.8 \mathrm{~K}$. Scaling the costs due to changing field at constant temperature is more complicated.

A summary of the costs associated with producing a $14.98 \mathrm{~m}$ magnetic length SSC Collider dipole at Fermilab ${ }^{27}$ shows the following rounded off costs:

Collar Parts

Yoke \& Skin

Collaring \& Final Assembly

Cryostat

Miscellaneous

Total M\&S
$60 \mathrm{K \$}$

30

54

75

10

$229 \mathrm{KS}$

27 Provided by G. Kobliska, 30 January, 1993.

Options for the Tevatron Tunnel, December 23, 1993 Page 38 
In addition, there is the labor to assemble the magnets at IB1 which was approximately $200 \mathrm{~K} \$$ per magnet. The labor cost could conceivably be reduced by a factor of two during a long production run. The labor costs do not include EDIA or magnet testing at MTF.

We also have a cost estimate made for LRIP (Low Rate Initial Production) for a 500 magnet production run 28 as follows:

Material

Labor

G\&A

Shipping

Conductor

Total including labor
$\$ 141 \mathrm{~K}$

$25 \mathrm{~K}$

$52 \mathrm{~K}$

$2 \mathrm{~K}$

$50 \mathrm{~K}$

$\$ 270 \mathrm{~K}$

The following magnet costs for $15 \mathrm{~m}$ magnetic dipoles, arbitrarily using $229 \mathrm{~K} \$$ and $100 \mathrm{~K} \$$ for the material and labor costs of a $15 \mathrm{~m}$ SSC Collider dipole as reference are:

Dipole

$15 \mathrm{~m}, 5 \mathrm{~cm}, 6.6 \mathrm{~T}$ SSC Collider

$15 \mathrm{~m}, 5 \mathrm{~cm}, 8.8 \mathrm{~T}$

$15 \mathrm{~m}, 7 \mathrm{~cm}, 6.6 \mathrm{~T}$

$15 \mathrm{~m}, 7 \mathrm{~cm}, 8.8 \mathrm{~T}$

$15 \mathrm{~m}, 5 \mathrm{~cm}, 8.8 \mathrm{~T}$ LHC type

$\begin{array}{lll}\text { Material } & \text { Labor } & \text { Total } \\ \text { K\$ } & \text { K\$ } & \text { K\$ } \\ 229 & 100 & 329 \\ 305 & 100 & 405 \\ 321 & 100 & 421 \\ 428 & 100 & 528 \\ 484 & 150 & 634\end{array}$

The LHC magnet cost scaling assumed that the material costs scaled as twice the coil cost of a single $5 \mathrm{~cm}$ and $8.8 \mathrm{~T}$ coil, twice the collaring, 1.5 times the yoke and skinning, equal the cryostat, and 1.5 the labor.

The new dipoles in the tunnel have a nominal magnetic length of $12 \mathrm{~m}$. The above listed material costs should therefore be scaled down by the ratio $12 / 15$. This is done in the following table.

28 Report DOE/ER/0594P, Pg.87.

Options for the Tevatron Tunnel, December 23, 1993 Page 39 


$\begin{array}{llll}\text { Dipole } & \text { Material } & \text { Labor } & \text { Total } \\ & \mathrm{K} \$ & \mathrm{K \$} & \mathrm{K \$} \\ 12 \mathrm{~m}, 5 \mathrm{~cm}, 6.6 \mathrm{~T} \text { SSC Collider } & 183 & 100 & 283 \\ 12 \mathrm{~m}, 5 \mathrm{~cm}, 7.7 \mathrm{~T} & 213 & 100 & 313 \\ 12 \mathrm{~m}, 5 \mathrm{~cm}, 8.8 \mathrm{~T} & 244 & 100 & 344 \\ 12 \mathrm{~m}, 6 \mathrm{~cm}, 6.6 \mathrm{~T} & & & \\ 12 \mathrm{~m}, 6 \mathrm{~cm}, 7.7 \mathrm{~T} & 220 & 100 & 320 \\ 12 \mathrm{~m}, 6 \mathrm{~cm}, 8.8 \mathrm{~T} & 257 & 100 & 357 \\ 12 \mathrm{~m}, 7 \mathrm{~cm}, 6.6 \mathrm{~T} & 293 & 100 & 393 \\ 12 \mathrm{~m}, 7 \mathrm{~cm}, 7.7 \mathrm{~T} & & & \\ 12 \mathrm{~m}, 7 \mathrm{~cm}, 8.8 \mathrm{~T} & 257 & 100 & 357 \\ 12 \mathrm{~m}, 5 \mathrm{~cm}, 8.8 \mathrm{~T} \text { LHC type } & 300 & 100 & 400 \\ & 342 & 100 & 442 \\ & 387 & 150 & 537\end{array}$




\subsection{Proton Proton Using 8.8 Tesla 2-in-1 Magnets}

This option requires two high field rings within the tunnel and an interaction region lattice design that brings the two beams into collision.

If two new rings are installed in the tunnel, we have the option of installing two vertically stacked separate rings or a single ring composed of two-in -one style magnets as the LHC dipole.

The LHC dipole option can have apertures that are small if the Tevatron ring is retained for fixed target physics. The detectors need not be raised in this case if the Tevatron is raised above the LHC style ring. As in the single ring proton-antiproton case, the peak operating field of the dual bore magnet is limited to approximately $6.6 \mathrm{~T}$ for $4.2 \mathrm{~K}$ operation and approximately $8.8 \mathrm{~T}$ for $1.8 \mathrm{~K}$ refrigeration. Collisions with horizontal crossings are easily achieved with dual dipoles but a new low beta lattice and/or stronger low beta quadrupoles will be required for beam energies above $1 \mathrm{TeV}$.

In order to have the two beams traverse the same circumference in one turn, it is required that the beams pass from the inner to the outer accelerator an even number of times. This can be done by having two interaction regions, for example. However, it is probably a good idea to have the beams pass through a common $\mathrm{RF}$ system. It is not necessary, nor desirable due to the additional beam beam tune shift, to have the beams collide in the RF system. If so, one must have a fourth location where the beams exchange accelerators.

\subsection{Energy}

A dipole field of 8 Tesla gives $\mathrm{E}_{\mathrm{Cm}}=(8 / 4.4) \times 2 \mathrm{TeV}=3.64$ TeV.

\subsection{Energy Limitations}

Although it not thought to be as difficult to bring the beams into collision as with other proton proton options, it still has to be shown to be possible in within the constraints of the Tevatron tunnel. 
For the purposes of comparison to the proton antiproton option, a center of mass energy of $4 \mathrm{TeV}$ will be chosen.

\subsection{Crossing Angle}

For 1 bucket spacing, a crossing angle is required. For the 2-in1 magnet design, a horizontal angle is the natural choice.

\subsection{Luminosity, Event Rates, and Bunch Spacing}

As shown in Tables $1 \mathrm{~b}$ and $2 \mathrm{~b}$, a proton proton collider operating at $\mathrm{E}_{\mathrm{cm}}=4 \mathrm{TeV}$ can produce about $2 \times 10^{33} \mathrm{~cm}^{-2} \mathrm{sec}^{-1}$, and provide $403 \mathrm{pb}^{-1}$ per week. It is expected ${ }^{29}$ to produce about:

4000 top pairs per week assuming mtop $=200 \mathrm{GeV}$, and 36,000 top pairs per week assuming $m_{\text {top }}=140 \mathrm{GeV}$.

The bunch spacing for this collider option is $18.83 \mathrm{nsec}$, and the number is interactions per crossing is expected to be 2.77. The present plans for the CDF and D0 detector upgrades will not accommodate this. However, this bunch spacing is similar to those planned for the SSC / LHC, and the detectors were / are being designed to handle this event rate.

\subsection{Luminosity Limitations}

The luminosity is limited by the Main Injector proton brightness $N / \varepsilon$. It is a factor of about 4 from being limited by the beam beam tune shift.

The integrated luminosity is limited by the interaction rate of the protons being used to create collisions for high energy physics. One would fill the proton proton collider more often than the antiproton proton collider, since the limit on antiproton production does not exist.

29 Hill and Parke, ibid. 


\subsection{Dipole Sagitta}

The magnets will be designed so that the sagitta is OK.

\subsection{Magnet Aperture}

Magnets for a proton proton collider do not face the same aperture requirements as an antiproton proton collider because the proton beams can be injected into the center of the magnet aperture.

However, the $50 \mathrm{~mm}$ aperture of the SSC magnets was considered barely adequate for the SSC beam parameters. The SSC design transverse emittance was a factor of four smaller than that to be provided by the Main Injector. In addition, the SSC lattice provided beta functions which were smaller than the Tevatron lattice, and the energy was a factor of two higher. Thus, the Fermilab beam size is at least twice as large as the SSC design. This is presents a technical risk for the use of $50 \mathrm{~mm}$ magnets at Fermilab.

\subsection{Resonant Extraction}

These magnets can probably support resonant extraction. See Section 1.8 for discussion on this point.

\subsection{Fixed Target Ramp Rate}

Designing these magnets to accommodate fixed target ramping rates may prove to be a problem.

\subsection{Cryostat}

The outer vacuum vessel fits in the Tevatron tunnel fine. The detectors would have to move up slightly.

\subsection{Cryogenics Requirements}


The present Fermilab cryogenic system can provide $3.5^{\circ} \mathrm{K}$. To achieve 8.8 Tesla these magnets would require $1.8^{\circ} \mathrm{K}$.

\subsection{Installation and Commissioning}

The installation and commissioning would not have considerations different from those discussed in Section 1.12.

\subsection{Elements of Cost}

This proton proton option is more expensive than the antiproton proton option and it poses some significant additional technical risks as a project. However, it does produce about 10 times as many tops per week as the antiproton proton option, and is a first class top factory.

The elements of the cost can be divided into four parts: $R \& D$, production, installation, and operation.

R\&D on 2-in-1 superconducting devices for $1.8^{\circ} \mathrm{K}$ :

8.8 Tesla dipoles, lattice and straight section quads, low beta quads

R\&D on conventional devices:

beam dump kickers and beam absorber

R\&D on lattice design

R\&D on a detector to handle $18.83 \mathrm{nsec}$ bunch spacing

Development of lattice engineering layout

production of 38412.5 meter lattice dipoles

production of 6 "half dipoles" as necessary for the 48 locations.

production of about 200 lattice quads of two types

production of straight section quads of three types

production of about 200 correction packages of many types

production of low beta quads

production of kickers and dump for beam dump if necessary

installation of superconducting ring and connection to cryogenics

operation of 1.8 degree cryogenics for low beta inserts 


\subsection{Proton Proton Using Tevatron and A New Ring}

This option requires two high field rings within the tunnel and an interaction region lattice design that brings the two beams into collision.

This option, $1 \mathrm{TeV}$ protons on $1 \mathrm{TeV}$ protons, retains the Tevatron magnets and adds a second ring at the approximate location of the removed conventional Main Ring. This option has been covered in the Fermilab conceptual design report "Proton-Proton Collider Upgrade", May 1988.

The Tevatron would continue as the fixed target accelerator with slow extraction. The SSC Collider dipole operated at 4.4 T maximum could form the upper ring. The same dipole, operated at $6.6 \mathrm{~T}$, can be used to make room for the vertical bends required at both sides of each interaction region. This option does not require a major refrigerator upgrade, utilizes the same lattice in the upper and lower ring and essentially the same low beta insertions. It does require that the detectors be raised to accommodate the raised collision point elevation and requires a major upgrade in the collider detectors to utilize the higher event rates required if this option is to produce a higher top production rate than will be available after the Main Injector upgrade.

In this option, if the RF cavities are shared between the two accelerators when in collider mode, it will have to be reinstalled in the Tevatron for fixed target to maintain an acceptable ramp rate.

As with the antiproton proton option, this proton proton option only needs one new ring. However, it is not as efficient in producing tops. As Tables 1 and 2 show, one produces more luminosity with the proton proton option, but fewer tops. In addition to larger backgrounds in the detectors, one also produces more radiation on the vertex detectors per top. Also the number of interactions per crossing is much larger (as much as 17) and the top production is still smaller. These taken together indicate that the antiproton proton option is a better choice for studying the top than this proton proton option. 


\subsection{Energy}

The center of mass energy is limited to $2 \mathrm{TeV}$ since the Tevatron can only go to $1 \mathrm{TeV}$. The new ring need only need go to 1 $\mathrm{TeV}$ but it would probably be designed to allow higher energy. Beams can be brought into collision within the constraints of the Tevatron tunnel using 6.6 Tesla dipoles.

\subsection{Energy Limitations}

The present Tevatron limits the center of mass energy to 2 TeV.

\subsection{Crossing Angle}

Table $1 \mathrm{~b}$ shows both a 7 bucket spacing and a 1 bucket spacing option. For the 1 bucket spacing the discussion is similar to that in section 2.3 except the natural crossing angle may not be horizontal since the accelerators are on spaced vertically. For the 7 bucket spacing, one may be able to get the beams separated more easily using magnets rather than electrostatic separators as discussed in section 1.3.

\subsection{Luminosity, Event Rates, and Bunch Spacing}

As shown in Tables $1 \mathrm{~b}$ and $2 \mathrm{~b}$, a proton proton collider operating at $\mathrm{E}_{\mathrm{cm}}=2 \mathrm{TeV}$ can produce about 1 to $2 \times 10^{33} \mathrm{~cm}^{-2} \mathrm{sec}^{-}$ 1 , and provide 200 to $400 \mathrm{pb}^{-1}$ per week. It is expected ${ }^{30}$ to produce about:

100 to 200 top pairs per week assuming $m_{t o p}=200 \mathrm{GeV}$, and 1800 to 3600 top pairs per week assuming mtop $=140 \mathrm{GeV}$.

The bunch spacing for this collider option is either $18.83 \mathrm{nsec}$ or $132 \mathrm{nsec}$. For the former the number of interactions per crossing would be 1.23, and for the latter it would be 17.6. The present plans for the $\mathrm{CDF}$ and $\mathrm{D} 0$ detector upgrades will not accommodate the

30 Hill and Parke, ibid.

Options for the Tevatron Tunnel, December 23, 1993 Page 46 
18.83 nsec spacing, and it is not obvious how the detectors would handle 18 interactions per crossing. However, this bunch spacing is similar to that planned for the LHC, and the detectors are being asked to handle 20 interactions per crossing.

\subsection{Luminosity Limitations}

The luminosity limitations are similar to those discussed in section 2.5 and is limited by the Main Injector proton brightness $\mathrm{N} / \varepsilon$. For the 1 bucket spacing, it is a factor of about 4 from being limited by the beam beam tune shift. However, for the 7 bucket spacing it is near the limit.

The integrated luminosity is limited by the interaction rate of the protons being used to create collisions for high energy physics. One would fill the proton proton collider more often than the antiproton proton collider, since the limit on antiproton production does not exist.

\subsection{Dipole Sagitta}

The magnets will be designed so that the sagitta is OK.

\subsection{Magnet Aperture}

The new ring can be designed as a collider and the beams can be injected into the center of the magnet aperture. However, the discussion about the $50 \mathrm{~mm}$ aperture in section 2.7 still applies.

\subsection{Resonant Extraction}

The new magnets do not have to support resonant extraction since the Tevatron would be retained.

\subsection{Fixed Target Ramp Rate}

The new magnets do not have to support resonant extraction since the Tevatron would be retained. 


\subsection{Cryostat}

The outer vacuum vessel fits in the Tevatron tunnel above the Tevatron. The detectors would have to move up about 12 inches. The situation in F-sector becomes complicated by the presence of the Main Injector beam transfer line. The elements in the upper superconducting accelerator probably become trapped by the conventional magnets in the beam line.

\subsection{Cryogenics Requirements}

The present cryogenic plant is matched to the Tevatron. Adding another accelerator will require an upgraded cryogenics system.

\subsection{Installation and Commissioning}

The installation and commissioning would not have considerations different from those discussed in Section 1.12.

\subsection{Elements of Cost}

This proton proton option is probably not more expensive than the antiproton proton option and it does not pose significant technical risks as a project. However, it does produce about 10 times the luminosity as the Main Injector. However, for top production it is not as good as the antiproton proton option already discussed.

The elements of the cost can be divided into four parts: R\&D, production, installation, and operation.

$\mathrm{R} \& \mathrm{D}$ on a detector to handle $18.83 \mathrm{nsec}$ bunch spacing in one case Development of superconducting devices for $3.5 \% \mathrm{~K}$ : 6.6 Tesla dipoles, lattice and straight section quads Development of conventional devices: beam dump kickers and beam absorber

Development of lattice engineering layout production of 38412.5 meter lattice dipoles 
production of 6 "half dipoles" as necessary for the 48 locations. production of about 200 lattice quads of two types production of straight section quads of three types production of about 200 correction packages of many types production of low beta quads production of kickers and dump for beam dump if necessary installation of superconducting ring and connection to cryogenics operation of 1.8 degree cryogenics for low beta inserts 


\subsection{Proton Proton Using SSC Magnets}

This option requires two high field rings within the tunnel and an interaction region lattice design that brings the two beams into collision.

If two new rings are installed in the tunnel, we have the option of installing two vertically stacked separate rings or a single ring composed of two-in-one style magnets as the LHC dipole.

There is insufficient room in the tunnel for the horizontal placement of two separate rings. At least one of the vertically stacked separate rings must have sufficient aperture for slow extraction if the fixed target program is to be retained. The detectors will have to be raised because of the higher collision point. This option also requires space for vertical dipoles to bring the beams into collision and for beam energies higher than $1 \mathrm{TeV}$, a new low beta system with rearranged lattice and/or stronger quadrupoles.

This option could evolve from the antiproton proton option by the addition of a second ring and provide higher luminosities. However, spending money on this second ring should be considered in competition with providing a better antiproton source.

\subsection{Energy}

The center of mass energy would be the same as the antiproton proton collider if the two proton beams could be brought into collision.

\subsection{Energy Limitations}

The ability to bring the proton beams into collision and provide a low beta insert will limit the energy.

\subsection{Crossing Angle}

The issues are the same as discussed in section 3.3. 


\subsection{Luminosity, Event Rates, and Bunch Spacing}

The issues are the same as discussed in section 3.4.

\subsection{Luminosity Limitations}

The issues are the same as discussed in section 3.5.

\subsection{Dipole Sagitta}

The magnets will be designed so that the sagitta is OK.

\subsection{Magnet Aperture}

The issues are the same as discussed in section 3.7.

\subsection{Resonant Extraction}

The issues are the same as discussed in section 2.8.

\subsection{Fixed Target Ramp Rate}

The issues are the same as discussed in section 1.9.

\subsection{Crvostat}

The issues are the same as discussed in section 3.10.

\subsection{Cryogenics Requirements}

The present cryogenic system can probably handle two new accelerators since the heat leak would be much smaller than that from the Tevatron. 


\subsection{Injector Issues}

\subsection{Bunch Length and Intensity}

The section calculates the intensities and bunch lengths resulting from the standard 21 bucket coalescing as well as the 7 bucket coalescing required to provide $132 \mathrm{nsec}$ collider bunch spacing.

Figure 5.1a contains a plot of the rotation RF voltages with unit fundamental $\mathrm{RF}$ voltages at $2.5 \mathrm{MHz}$ (21 bucket coalescing) or 7.5 $\mathrm{MHz}$ ( 7 bucket coalescing) and a 2nd harmonic voltage which is $-1 / 5$ the amplitude of the fundamental to linearize the wave in the region of the bunches. The 13 bunches of 21 bucket coalescing are also shown. Note that 5 bunches can be rotated by 7 bucket coalescing in the same region of linearity as used in the 21 bucket coalescing case.

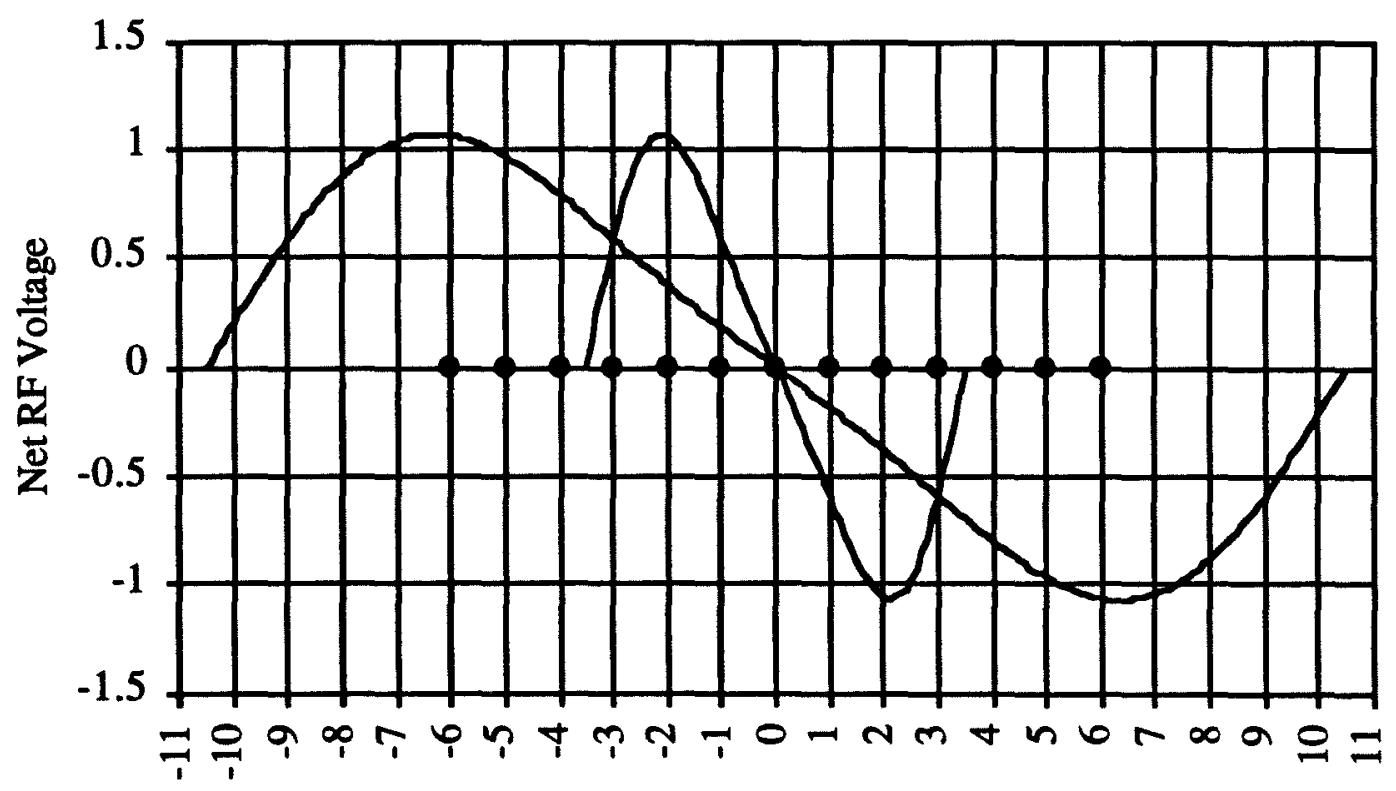

$53 \mathrm{MHz}$ RF Bucket

Figure 5.1a: Rotation RF voltages vs bucket number for both 21 and 7 bucket coalescing. The 13 bunches rotated during 21 bucket coalescing are also shown.

\subsubsection{Bucket (High Intensity) Calculation}

Options for the Tevatron Tunnel, December 23, 1993 Page 52 
The following calculation assumes 13 bunches, since increasing to 15 bunches only yields a small intensity increase. The assumed initial bunch parameters are longitudinal emittances of $0.2 \mathrm{eV}-\mathrm{sec}$ and intensities of $6 \times 10^{10}$. Since Main Injector era parameters are assumed, the rotation voltage is assumed to be $60 \mathrm{kV}$ at $2.5 \mathrm{MHz}$.

The first stage of coalescing is the minimization of the momentum spreads of the 15 bunches. Assuming perfectly adiabatic debunching into sinusoidal $53 \mathrm{MHz} \mathrm{RF}$ bucket the bucket half height would be $8.3 \mathrm{MeV}$. The equation relating voltage and bucket half height is

$$
\Delta E_{1 / 2}=\sqrt{\frac{2 V_{o} E_{o}}{\pi \eta h}}
$$

The bucket area associated with this bucket height is

$$
A=\frac{8}{\omega_{R F}} \Delta E_{1 / 2}
$$

Snap coalescing plus a second harmonic cavity can be used to generate a bunch phase space distribution with a $95 \%$ bunch half height of approximately $10 \mathrm{MeV}$. The net $95 \%$ invariant longitudinal emittance associated with this full phase space distribution is roughly the full height times 13 times the RF bucket length, or 4.9 $\mathrm{eV}$-sec. Note that the theoretical total emittance of $13 \times 0.2=2.6 \mathrm{eV}$ sec is not achieved since there are still spaces between the bunches which are not filled with particles.

To zeroth order, bunch rotation by the $2.5 \mathrm{MHz} \mathrm{RF}$ system preserves this emittance when the linearizing $2 \mathrm{nd}$ harmonic $\mathrm{RF}$ voltage is tuned to the optimum value. The $60 \mathrm{kV}$ is enough to guarantee that the beam distribution is completely contained within the central $53 \mathrm{MHz}$ recapture bucket. The central bunch simply spins around itself, trading off initial momentum spread for final bunch length after $90^{\circ}$ of synchrotron oscillation according to the equation 


$$
\Delta \tau=\frac{\eta}{E_{0} \Omega_{s}} \Delta E
$$

where

$$
\Omega_{s}=\sqrt{\frac{2 \pi h \eta V_{0}}{E_{o}}} \frac{f_{o}}{\beta_{r}}
$$

For $60 \mathrm{kV}$, angular synchrotron frequency $\Omega_{\mathrm{s}}$ is $30 \mathrm{rad} / \mathrm{sec}$ and the half height of $10 \mathrm{MeV}$ turns into a momentum half spread of $7 \mathrm{nsec}$. Simultaneously, the time length of the bunches is converted into a momentum half spread of $200 \mathrm{MeV}$.

To recapture the rotated charge distribution, the bucket height of the recapturing $53 \mathrm{MHz} \mathrm{RF}$ must exceed the momentum distribution. More importantly, the voltage should be set such that the bucket shape is matched to the shape of the charge distribution, thus minimizing emittance dilution due to phase space mismatch. Unfortunately, since the distribution is roughly uniform in the energy plane and approximately Gaussian in the time plane, and the general distribution is rectangularly shaped, some mismatch is inevitable. In the above case a final longitudinal emittance of approximately $5.5 \mathrm{eV}-\mathrm{sec}$ is expected.

After ramping the Tevatron to $1 \mathrm{TeV}$, the rms bunch length is calculated using equations (5.1.1c) and (5.1.1d) to be roughly $60 \mathrm{~cm}$ at an $\mathrm{RF}$ voltage of $1 \mathrm{MeV}$. Since the charge efficiency of coalescing should be $>90 \%$ in the Main Injector era (snap coalescing, 2 nd $h=2226$ linearization RF system, 20 to $60 \mathrm{kV}$ beam rotation $R F$ voltage upgrade), the total charge of the coalesced bunch is $700 \times 10^{9}$.

\section{1 .221 Bucket (Main Injector) Calculation}

In the standard Main Injector parameter set the proton intensity is estimated at $330 \times 10^{9}$. Assuming an efficiency of $90 \%$ and $60 \times 10^{9}$ protons per bunch means that the number of bunches coalesced is 7 . This reduced intensity was required due to the beambeam tune shift limit in the Tevatron Collider. 
The only parameter which changes in the rotation phase of coalescing is the longitudinal emittance of $2.6 \mathrm{eV}$-sec. Assuming a further dilution to $3 \mathrm{eV}-\mathrm{sec}$ due to recapture, the bunch length at 1 $\mathrm{TeV}$ in the Tevatron with a $1 \mathrm{MVolt} R \mathrm{RF}$ level is $45 \mathrm{~cm}$. The expected intensity at a coalescing charge efficiency of $90 \%$ is $380 \times 10^{9}$.

\section{1 .37 Bucket Calculation}

In this scenario 5 bunches is the maximum number which can be coalesced (see figure 5.1a). Since the longitudinal emittances of the bunches are the same as the 21 bucket coalescing case, the minimum momentum spread is also the same. But since there are fewer bunches, the momentum half spread and beam area of the charge distribution after rotation in a $20 \mathrm{kV} 7.5 \mathrm{MHz} \mathrm{RF}$ are $75 \mathrm{MeV}$ and $1.9 \mathrm{eV}$-sec. The diluted emittance after the process of recapture is estimated to be approximately 2.1 .

Therefore, after acceleration to $1 \mathrm{TeV}$ the bunch length of 35 $\mathrm{cm}$ is attainable. At an efficiency of $90 \%$, the total charge of the coalesced bunch is $270 \times 10^{9}$.

\subsubsection{Anticipated Coalescing Performance}

Though the numbers quoted in the above calculations are only approximate, they should be reasonably accurate for estimates of future injector performance after the commissioning of the Main Injector. The coalescing efficiencies quoted are based on present operational experience and straightforward and conservative estimates of the performance of coalescing upgrades already being acted on. In table 5.1.4 a compilation of the estimated performance numbers of coalescing under a number of scenarios are displayed. 
Table 5.1.4: Compilation of coalescing performance predictions in the era of Main Injector operations.

\begin{tabular}{|l|c|c|c|c|}
\hline & $\begin{array}{c}\text { No } \\
\text { Coalescing }\end{array}$ & $\begin{array}{c}\text { Full } \\
\text { Intensity } \\
21 \\
\text { Buckets }\end{array}$ & $\begin{array}{c}\text { MI } \\
\text { Params } \\
21 \\
\text { Buckets }\end{array}$ & $\begin{array}{c}\text { Full } \\
\text { Intensity } \\
7 \text { Buckets }\end{array}$ \\
\hline \hline Rotation Buckets & 1 & 21 & 21 & 7 \\
\hline $\begin{array}{l}\text { Number of } \\
\text { Bunches }\end{array}$ & 1 & 13 & 7 & 5 \\
\hline $\begin{array}{l}\text { Bunch Area (eV- } \\
\text { sec) }\end{array}$ & 0.2 & 5.5 & 3.0 & 2.1 \\
\hline Bunch Length (cm) & 10 & 60 & 45 & 35 \\
\hline $\begin{array}{l}\text { Final Intensity } \\
\left(x 10^{9}\right)\end{array}$ & 60 & 700 & 380 & 270 \\
\hline
\end{tabular}

\subsection{Counter-clockwise Protons in the Tevatron Tunnel.}

There are two options for injecting protons which travel counterclockwise in the Tevatron tunnel.

\subsubsection{Counter-clockwise in the Main Injector.}

In this option, protons are accelerated counter-clockwise in the Main Injector. They are transferred into the counter-clockwise ring in the Tevatron tunnel through a mile-long, essentially straight beamline from the MI-40 straight section to the Tevatron E-0 straight section. This option is obviously the simplest and probably the most expensive, unless the site between MI-40 and E- 0 is already developed as a new experimental area for $120 \mathrm{GeV}$ Main Injector extracted beamlines. Such an experimental area might need to be developed if new accelerators in the Tevatron tunnel displace the Main Ring remnant used for transporting beams to A0. If the area is developed, then the extension to E0 would be relatively short and simple.

\subsubsection{Clockwise in the Main Injector.}

In this option, protons are accelerated clockwise in the Main Injector and transferred to the Tevatron tunnel through the "A-150 line". This line was designed to transfer antiprotons into the Tevatron. While there is substantial effort required to install reversing switches on essentially 
every large power supply, the major difficulty in this option is injecting clockwise protons into the Main Injector. Three possible routes for the protons can be readily identified:

(i) From the Booster, protons are taken out the AP-4 line, through the Antiproton Source enclosures, down the AP-3 line and AP-1 line, through the Main Ring remnant from F-17 to F-0, and finally down the P-150 line. (Once the protons enter the AP-3 line, this is the same route antiprotons take from the Source to the Main Injector.) This solution is the least expensive, since no civil construction is required. It is undesirable in that the AP-4 line is restricted to low intensity due to the limited shielding, although that limitation either could be accepted or could be mitigated with additional shielding.

(ii) Construct a branch in the new Booster to Main Injector $8 \mathrm{GeV}$ line which connects into the AP-3 line, and then continues as in (i). The branch would require about 500 feet of new enclosure, plus the work on either end to connect to the $8 \mathrm{GeV}$ or AP-3 line. Both this approach and (i) require the Main Ring remnant to remain in place, which, as noted earlier, is a questionable assumption.

(iii) Construct a branch in the new Booster to Main Injector $8 \mathrm{GeV}$ line to connect into the Main Injector at MI-62. This branch would require more than 1000 feet of new enclosure, and the exact routing needs considerable discussion, due to the presence of the AP- 2 line, AP- 0 , etc. If the beamline for neutrino experiments is developed, some of that beamline enclosure (and even perhaps the beamline elements) could be common, so the actual injection could be in the MI-60 straight section. . However, without the neutrino line, vertical injection of $8 \mathrm{GeV}$ protons at MI-62 appears compatible with horizontal extraction to the Tevatron. The primary constraint is that the injection kicker must fit below the extraction C-magnet. 


\subsection{Issues to Be Addressed to The Director}

This section contains several issues that can be brought to the attention of the Director to impose additional constraints on the options discussed in this report.

\subsection{Physics Emphasis}

This report emphasizes top physics because Fermilab can exploit this physics in a timely fashion by choosing one of these accelerator options. However, it is the place of the PAC to formally advise the Director on such matters of physics. If a different physics emphasis were chosen, it is likely that a different set of accelerator options could emerge.

\subsection{Fixed Target}

This report emphasizes collider physics rather than fixed target physics. If the emphasis were reversed, perhaps different options would emerge.

Magnet designs which optimize collider operation result in requirements which conflict with requirements for an optimum fixed target magnet design. The electrical and cryogenics subsystem designs also are optimized differently for fixed target or collider operation. The following options are presented as alternatives to trying to design a magnet to do both.

The options presented above preserve the $120 \mathrm{GeV}$ fixed target option. However, in some options, the conventional $120 \mathrm{GeV}$ beam line in F-sector has to be mounted higher than the Main Ring level. In these cases:

a) the Main Injector has to match up to the new level,

b) the extraction from F17 must match the new higher level, and finally,

c) this $120 \mathrm{GeV}$ beam line must be brought down to Switchyard level in Transfer Hall.

However, the F-sector beam line does not have to be mounted over the present Main Ring accelerator, since it is no longer part of a 
closed accelerator. Finally, the conventional magnet string may "trap" the elements of the upper superconducting ring; if so, the conventional elements must be removed while replacing elements of the superconducting ring.

Using the Tevatron for $800 \mathrm{GeV}$ fixed target physics can be preserved in the proton antiproton options in one of two scenarios. In both cases, the Tevatron would be operated only for those months scheduled for fixed target physics, and the collider ring would be operated only for those months scheduled for collider physics. In the first scenario, the Tevatron would be left in place on the tunnel floor and the new collider ring would be mounted above it. This scenario requires moving the detectors at $\mathrm{BO}$ and $\mathrm{DO}$ up. In the second scenario, the new collider is placed on the floor and the Tevatron is remounted in a new position above it at approximately the old Main Ring level. This leaves the detectors approximately in place. In both scenarios, the Main Injector must match injection into the new locations.

\subsection{No New Beam Line Construction}

It has been assumed in this report that there will be no tunnel construction in addition to that which is presently part of the Main Injector project. Relaxation of this constraint leads to a proper proton beam transfer line for the proton proton option. In addition, such enclosures would relieve the congestion in F-sector where the $120 \mathrm{GeV}$ beam line shares a tunnel with a fixed target accelerator and a collider accelerator.

\section{No Improvements in the Antiproton Source}

No improvements in the Antiproton Source are included in this report beyond those required to allow full use of the Main Injector. The number of antiprotons could be increased by an order of magnitude before the beam beam tune shift limit in the collider is approached. One could expect to gain a factor of two in luminosity (and interactions per crossing) by adding a second source, for example.

\subsection{Interactions Per Crossing}

The number of interactions per crossing is seldom below 1 for the options in this report. One option has nearly 20 interactions per 
crossing. This may not too many for discovery of the top, but the following types of questions should be posed: "What physics cannot be done with multiple interactions per crossing?" and "Can precision top physics be done under these conditions?" It is not prudent to build an accelerator for which there is no detector to exploit its capabilities in a timely fashion.

\subsection{New Collider Tunnel}

The options appropriate for construction of a separate tunnel for the collider accelerator ${ }^{31}$ is presented here. The three main advantages of a separate tunnel are:

a) it allows access to larger energy, and thus more tops per week,

b) it allows year round high energy fixed target operation and collider operation,

and

c) it allows a collider lattice to be designed in which the low beta quads and devices for bringing beams into collision do not limit the energy more than the arc dipoles.

For reference, if a new tunnel were proposed, it could:

d) be 9 miles in circumference compared to the 4 mile Tevatron circumference,

e) house an accelerator based on 13 Tesla dipoles ${ }^{32}$ instead of 7.7 Tesla dipoles,

f) achieve luminosities which are about the same for the proton antiproton options (about $2 \times 10^{32} \mathrm{~cm}^{-2} \mathrm{sec}^{-1}$ ), but at least33 a factor of three higher for the proton proton options (at least 0.5 $x 10^{34} \mathrm{~cm}^{-2} \mathrm{sec}^{-1}$ ),

and

31 These options have been studied before under the names of site filler, Pentavac, Dedicated Collider, LINCOLN and site buster.

32 Perhaps these dipoles are similar to the 2-in 1 design described by $\mathrm{P}$. McIntyre of TAC at a Fermilab Accelerator Seminar, December 1993.

33 With two detectors and three abort gaps, proton proton accelerators confined to the Tevatron tunnel do not fill more than 756 of the 1113 buckets available. One could fill a larger fraction in the 9 mile ring. 
g) reach a center of mass energy of

$$
3.5 \mathrm{TeV} \times(13 / 7.7) \times(9 / 4)=13 \mathrm{TeV} .
$$

For reference ${ }^{34}$, the design for the $\mathrm{LHC}$ is $14 \mathrm{TeV}$ center of mass with luminosity of $10^{34} \mathrm{~cm}^{-2} \mathrm{sec}^{-1}$.

One set of choices for the accelerator parameters for these options are summarized in Table 1c. Table $2 \mathrm{c}$ contains the top production rates for such colliders. Table 6 summarizes calculations related to synchrotron radiation. Option PP13a puts 13 Tesla dipoles in the Tevatron tunnel; there is no known way to do the low beta for this option. Option PP13b puts the 13 Tesla dipoles in a new tunnel and achieves a center of mass energy of $13.5 \mathrm{TeV}$. Option PP13c reduces the bunch intensities until the synchrotron radiation power density is the same as in the LHC.

Table 6a.

Table 6b.

Total Power

Power Density

Energy Loss/Turn

Total Power

Power Density

Energy Loss/Turn
Synchrotron radiation

for antiproton proton options

compared to the Main Injector

$\begin{array}{rrrrc}\begin{array}{r}\text { Main } \\ \text { Injecter }\end{array} & \text { PA4 } & \text { PA6 } & \text { PA7 } & \text { PA8 } \\ & & & & \\ 0.8 & 1.2 & 6.2 & 11.5 & 19.7 \text { Watts } \\ 0.12 & 0.20 & 1.0 & 1.8 & 3.1 \mathrm{mWatts} / \mathrm{m} \\ 7.8 & 7.8 & 39 & 73 & 124 \mathrm{eV} / \text { particle }\end{array}$

Synchrotron radiation

for proton proton options

compared to the Main Injector

Main

Injector

$\begin{array}{llll}\text { PP4a PP4b } & \text { PP6 } & \text { PP8 }\end{array}$

0.8

5.4

3.5

27.3

86.2 Watts

0.1

0.9

0.6

4.3

$13.7 \mathrm{mWatts} / \mathrm{m}$

7.8

7.8

7.8

39.4

$124.4 \mathrm{eV} /$ particle

34 W. Scandale of CERN, talk presented at a Fermilab Accelerator Seminar, December 1993. 
Table 6c

\begin{tabular}{lrrrrl} 
& $\begin{array}{r}\text { Main } \\
\text { Injector }\end{array}$ & PP13a & PP13b & PP13c & \multicolumn{1}{l}{ LHC } \\
Total Power & 0.8 & 436 & 7006 & 2335 & 4383 Watts \\
Power Density & 0.1 & 69 & 495 & 165 & $162 \mathrm{mWatts} / \mathrm{m}$ \\
Energy Loss/Turn & 7.8 & 630 & 7173 & 7173 & $4347 \mathrm{eV} / \mathrm{particle}$
\end{tabular}

Of course, it is recognized that it takes money (billions of dollars):

h) to do the R\&D needed to achieve an inexpensive 13 Tesla dipole and corresponding quadrupoles and correction packages,

i) to build a tunnel with a 9 mile circumference,

j) to do the R\&D for new detectors and build new detectors,

k) to build a new accelerator (or two) which requires $1.8^{\circ} \mathrm{K}$ cryogens,

and

1) to run fixed target and collider year round. 


\section{Acknowledgments}

The principle authors of this report, David Finley and Gerry Jackson, enjoyed batting some of these ideas around with other members of the Accelerator Division. Much of the discussion, some of the text and some of the calculations were provided by members of the Accelerator Physics Department - headed by Pat Colestock including Alex Bogacz, Norman Gelfand, Karl Koepke, Leo Michelotti, Francois Ostiguy and Stan Pruss. Phil Martin also contributed some crucial pieces of text. Discussions with Dixon Bogert, Mike Church, John Marriner, Mike May, Bill Miller, Jay Theilacker and Dan Wolff brought some reality checks to some of the ideas dreamed up during this process. Finally, discussions with Chris Hill, Steve Parke and Alan Stange on the fundamental particle physics topics was a refreshing break for one of us (Finley).

Options for the Tevatron Tunnel, December 23, 1993 Page 63 


\section{List of Attachments}

MSD1

$\begin{array}{llll}M S D 2 a & 2 b & 2 c & 2 d\end{array}$

MSD3a $\quad 3 b \quad 3 c$

MSD1 thru 3

$\begin{array}{llllllllll}\text { MSD4a } & 4 b & 4 c & 4 d & 4 e & 4 f & 4 g & 4 h & 4 i & 4 j\end{array}$

Physics Sensitivity of Fermilab Machine Options

PH 

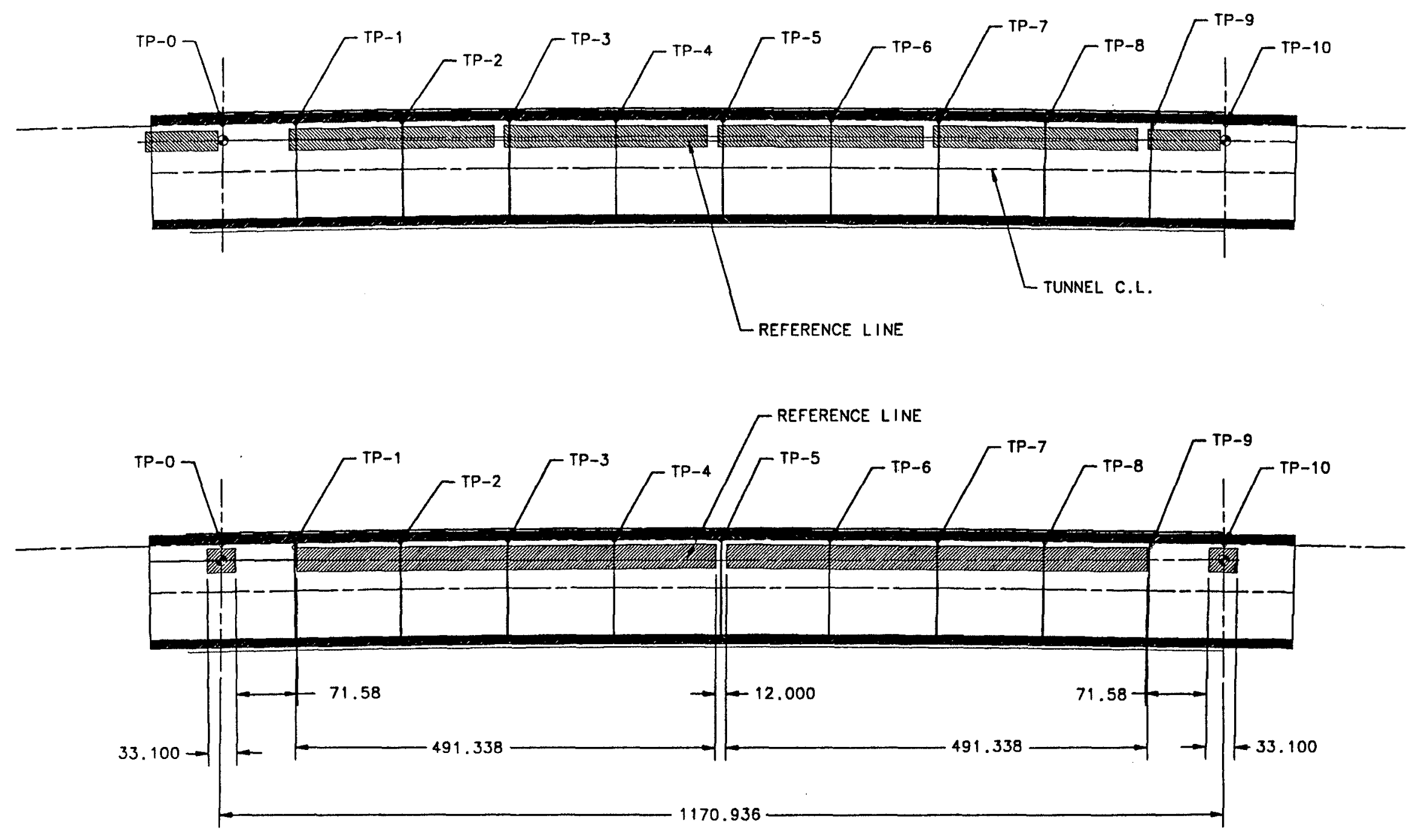

MSD 1 


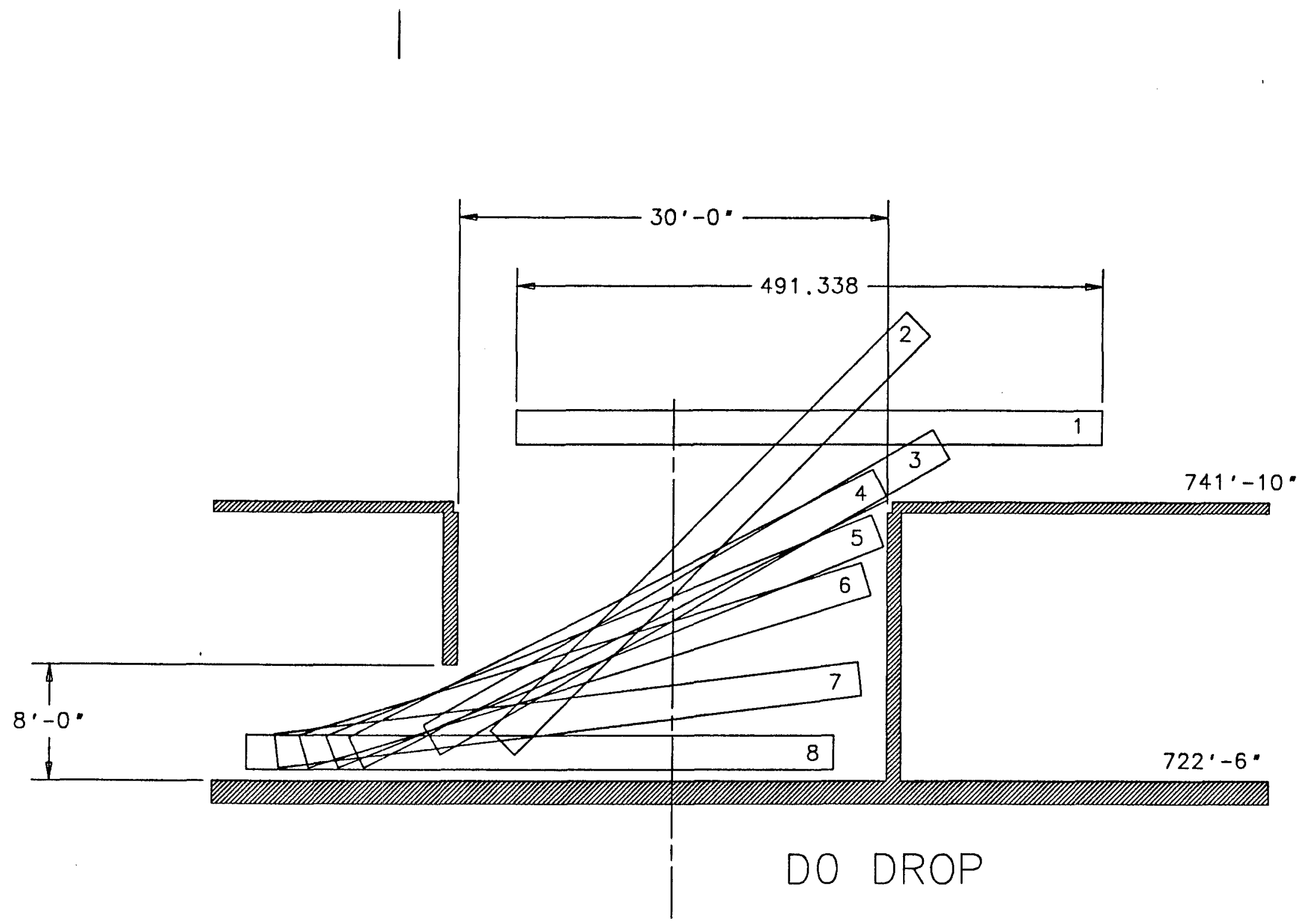

MSD Za 


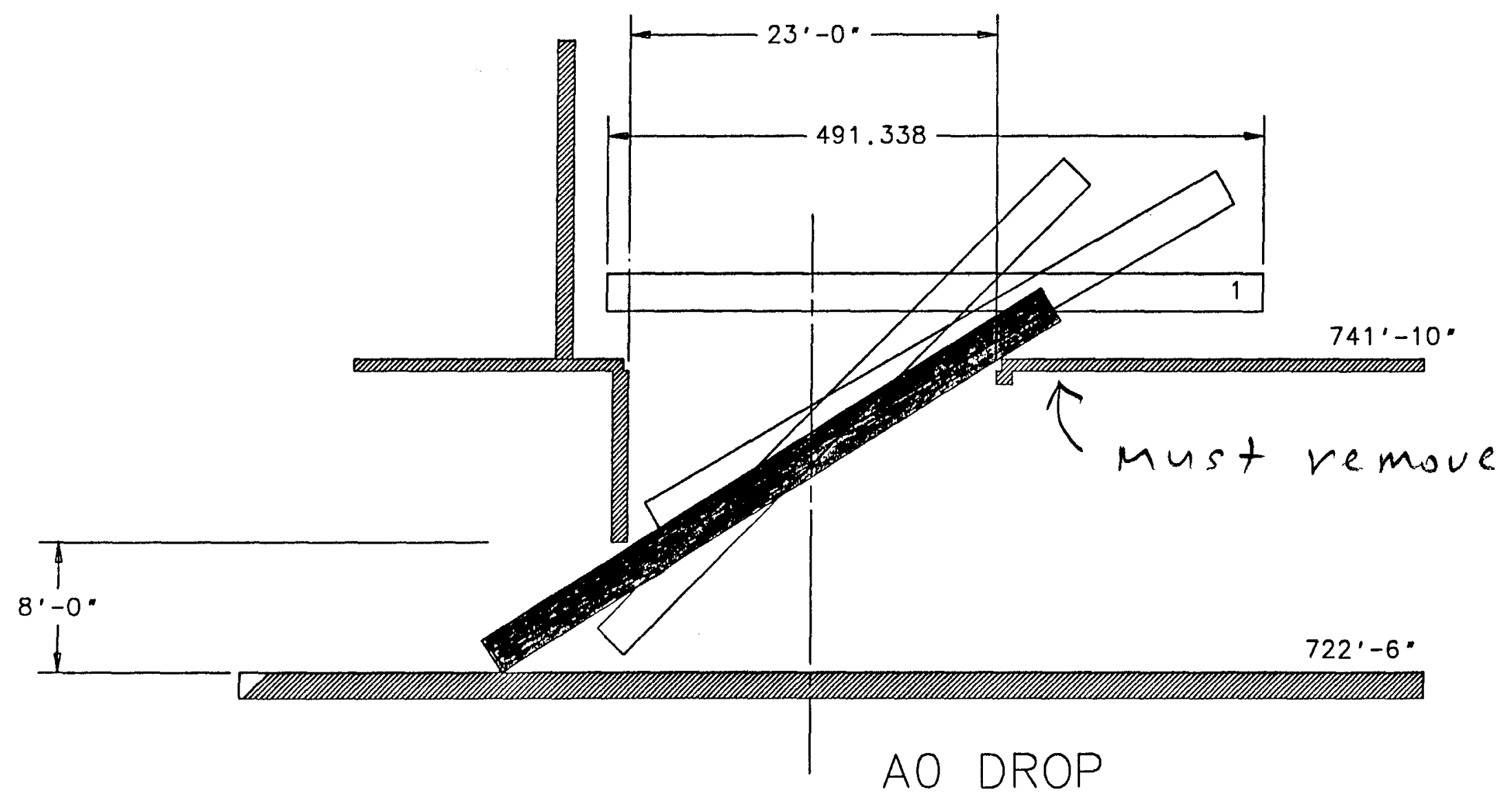

can we upend maguets

MSD 2b 


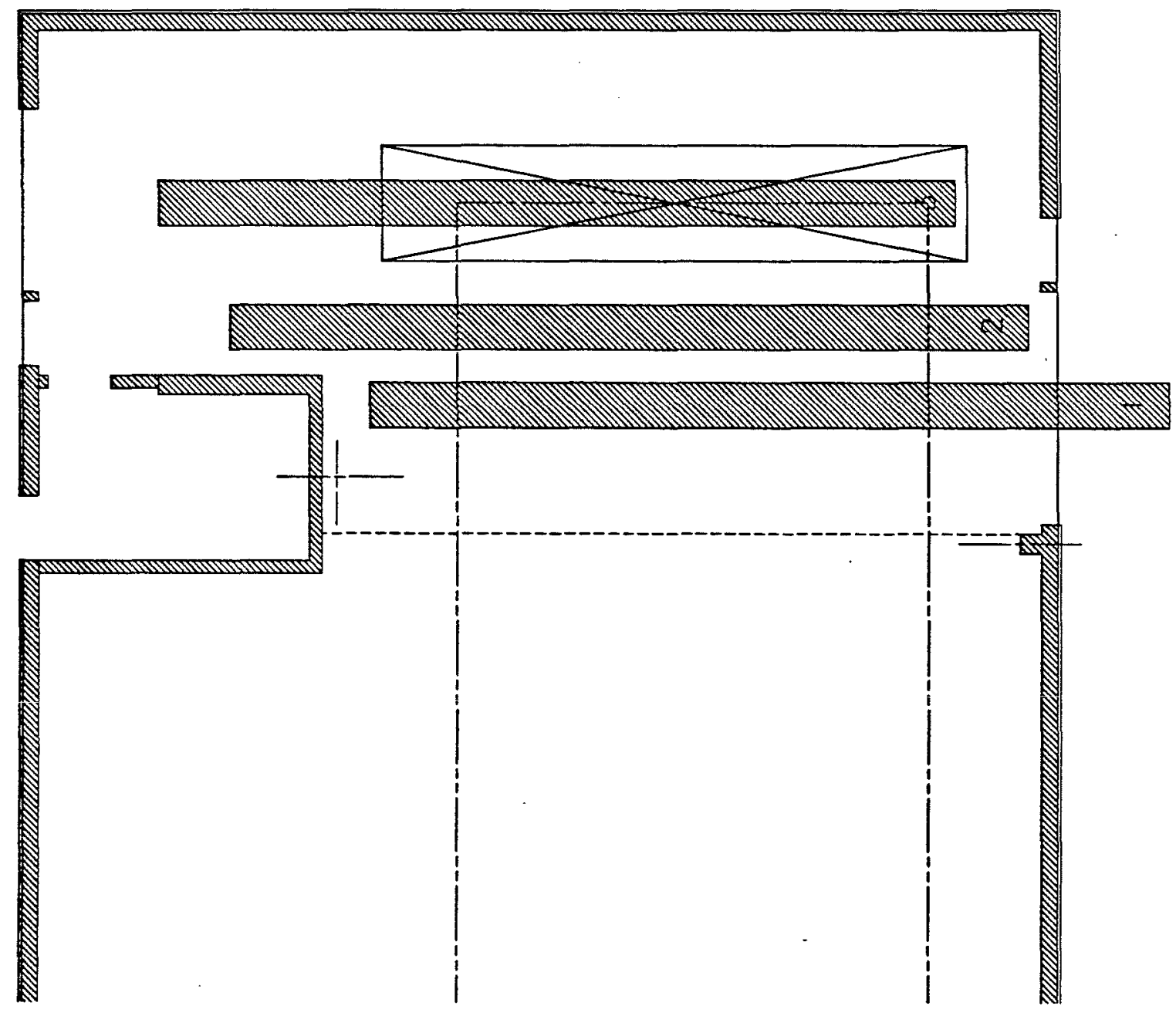

0
0
0
0
0
0

2
0
$n$
$N$




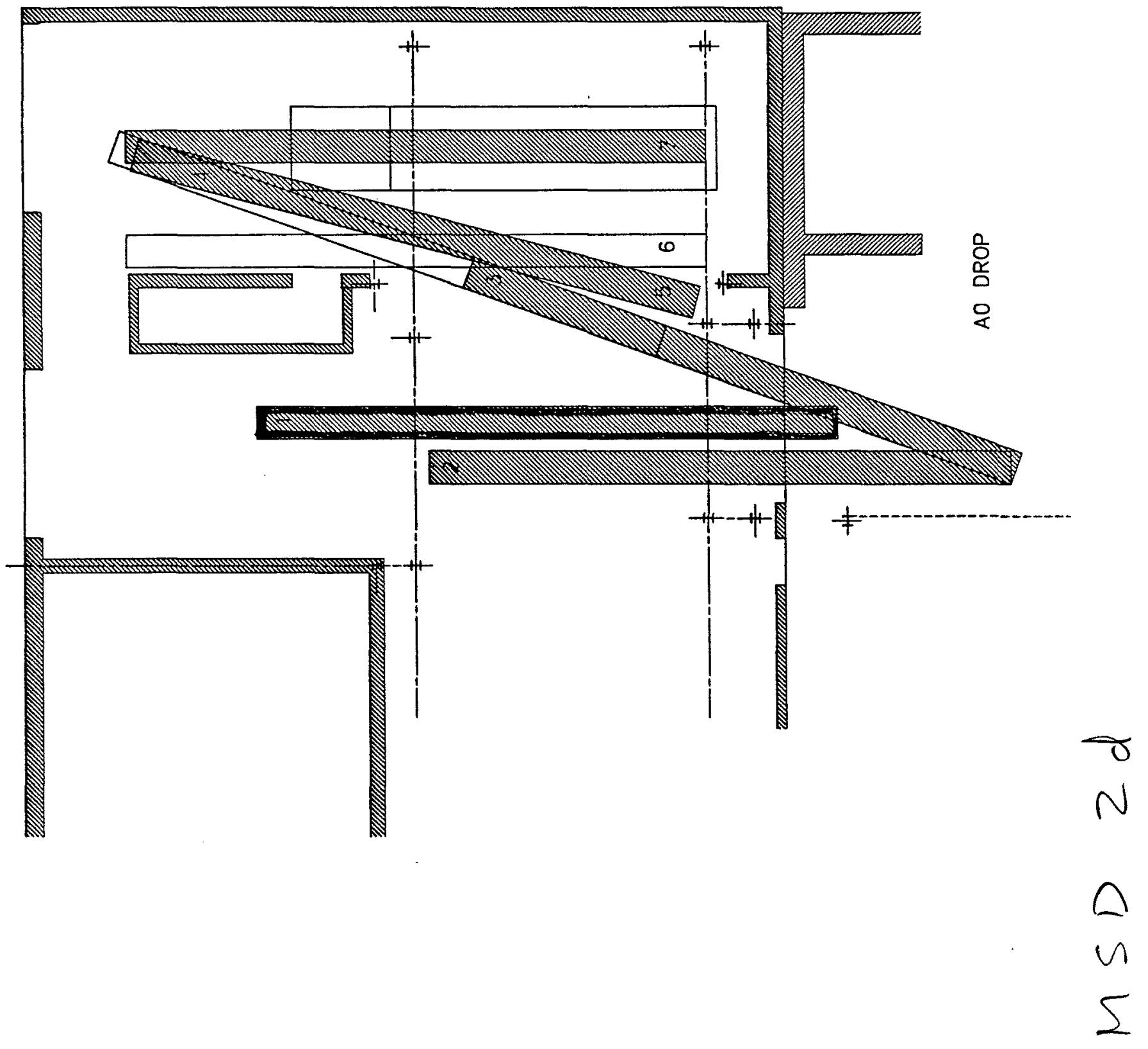



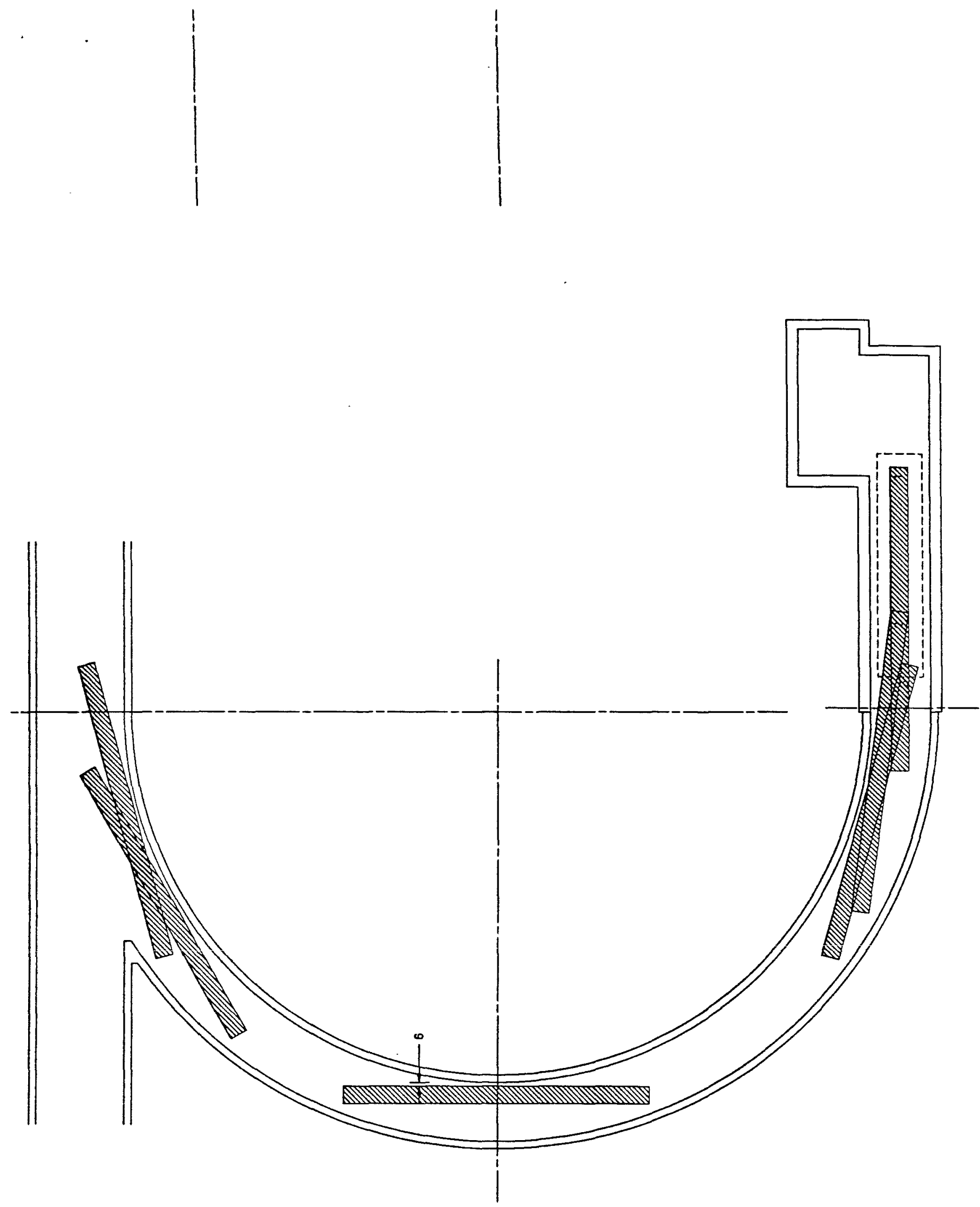

$\sigma$
$m$
$n$
$n$ 

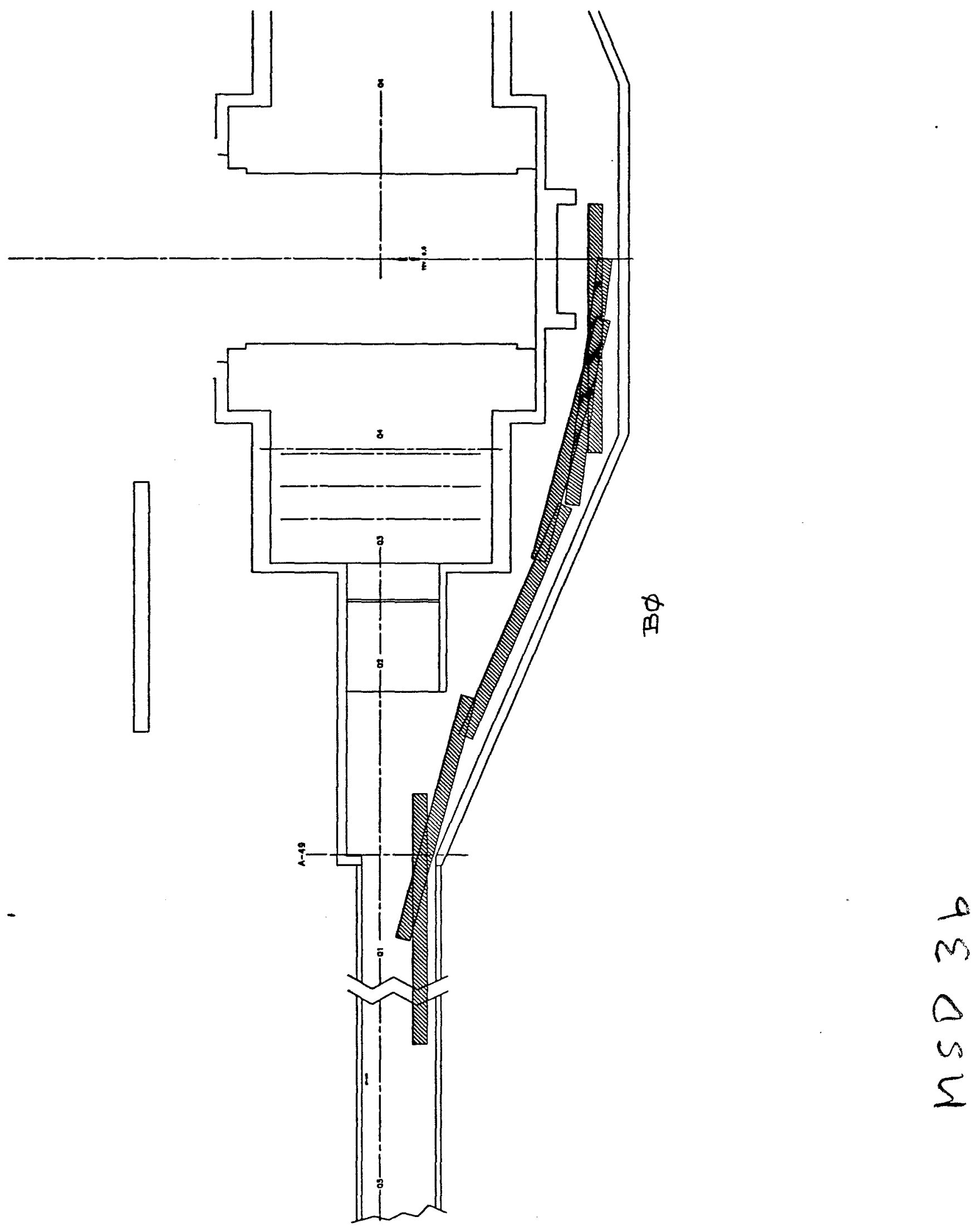


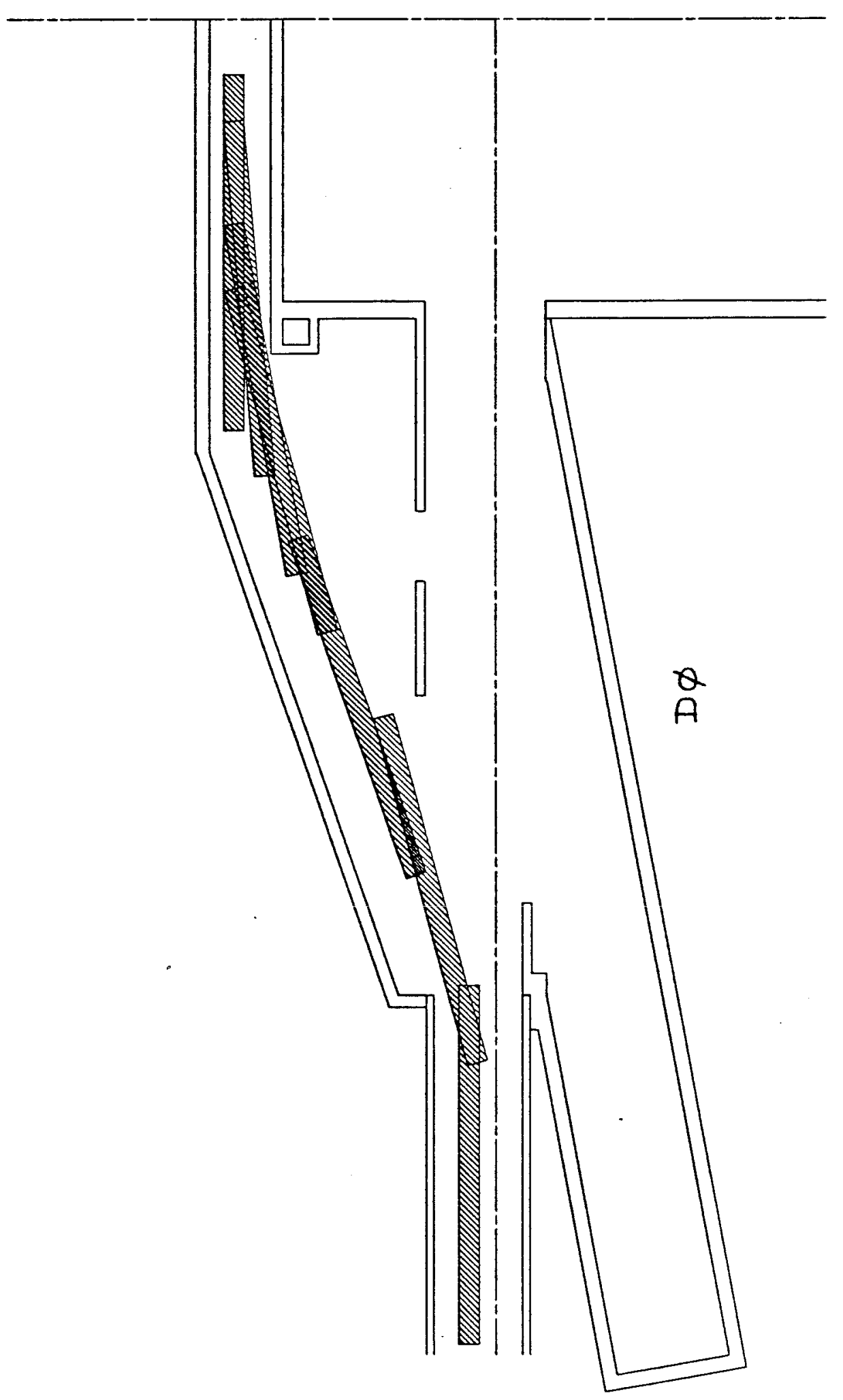

$u$
$n$
$\Sigma$ 

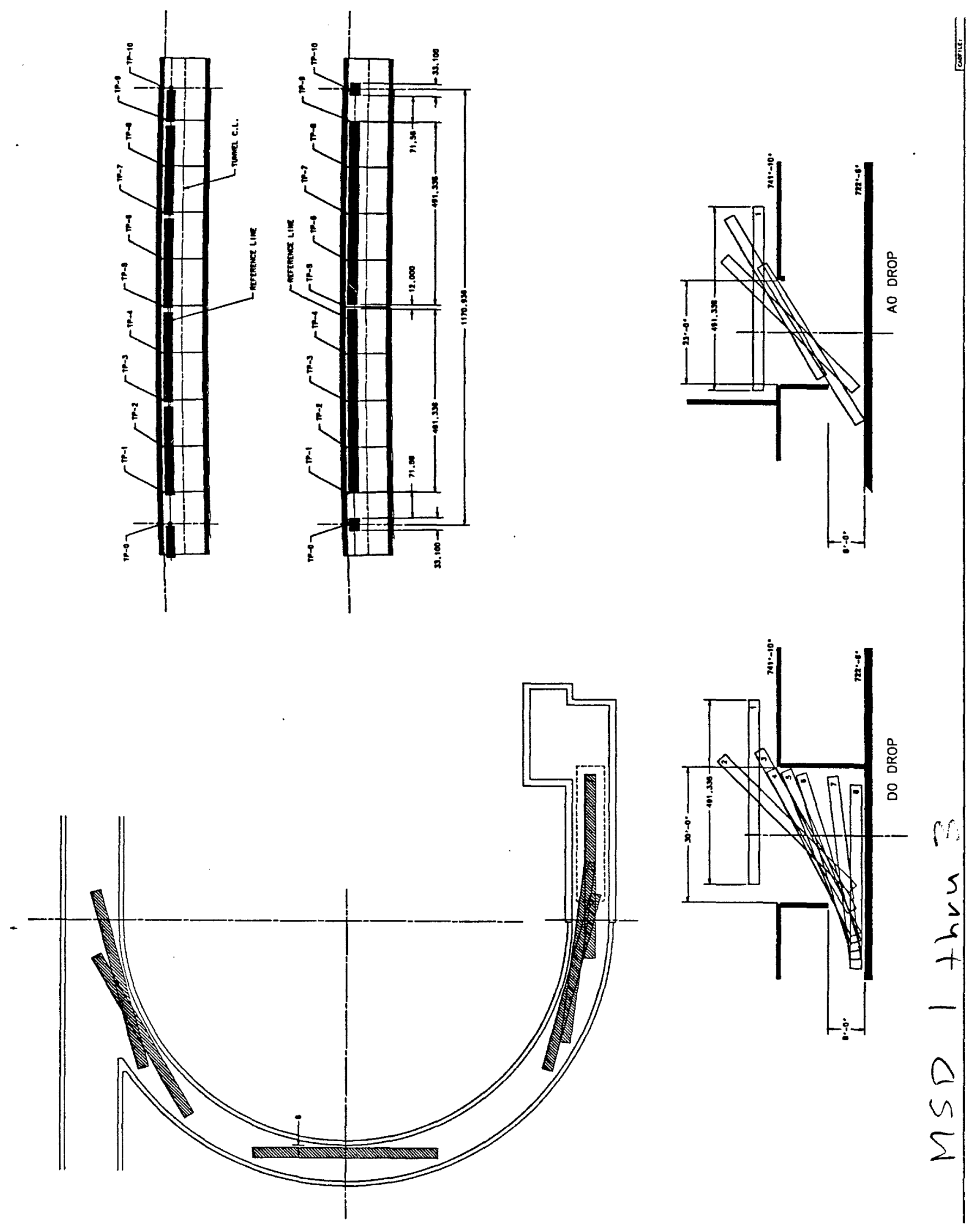


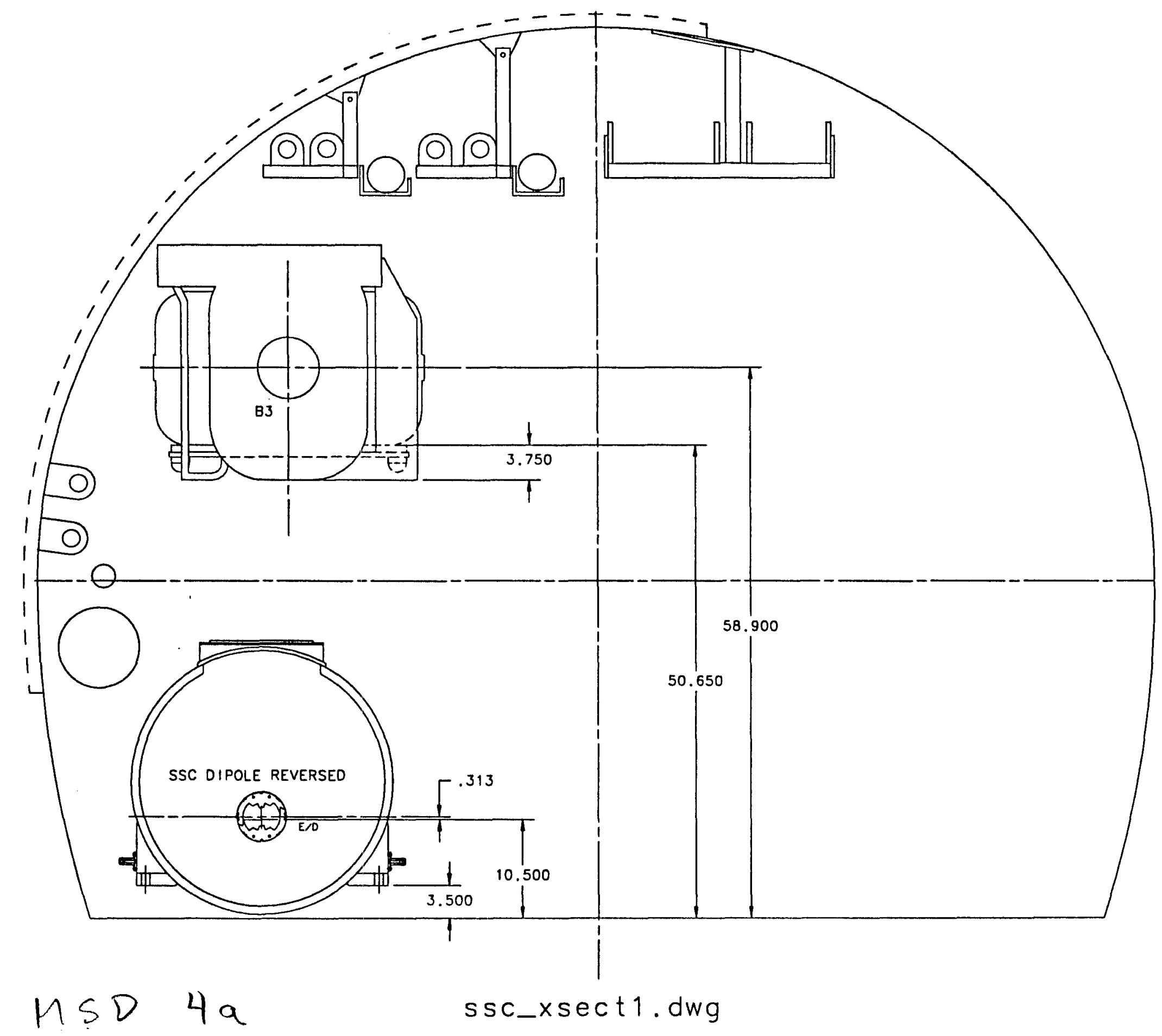




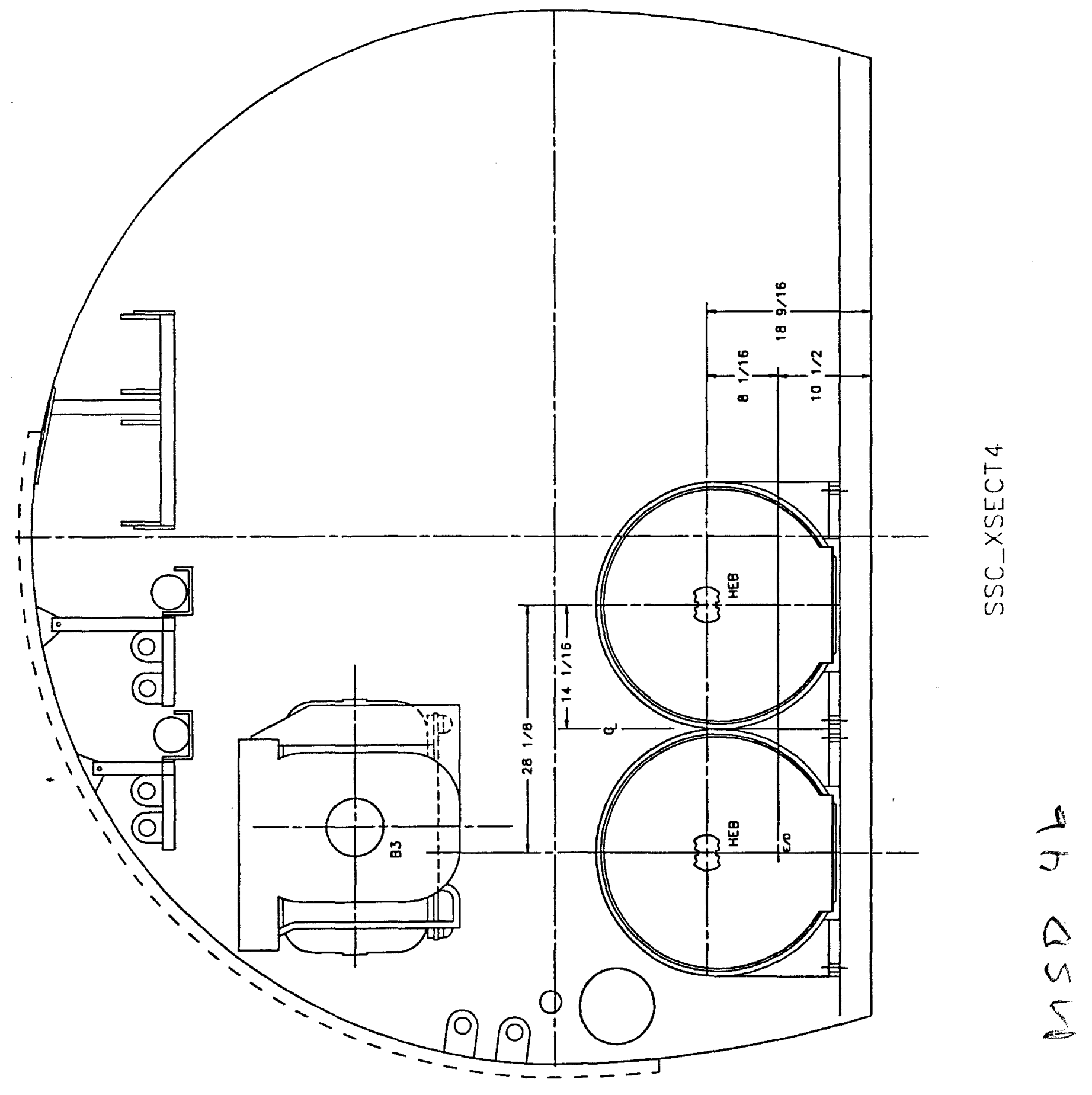




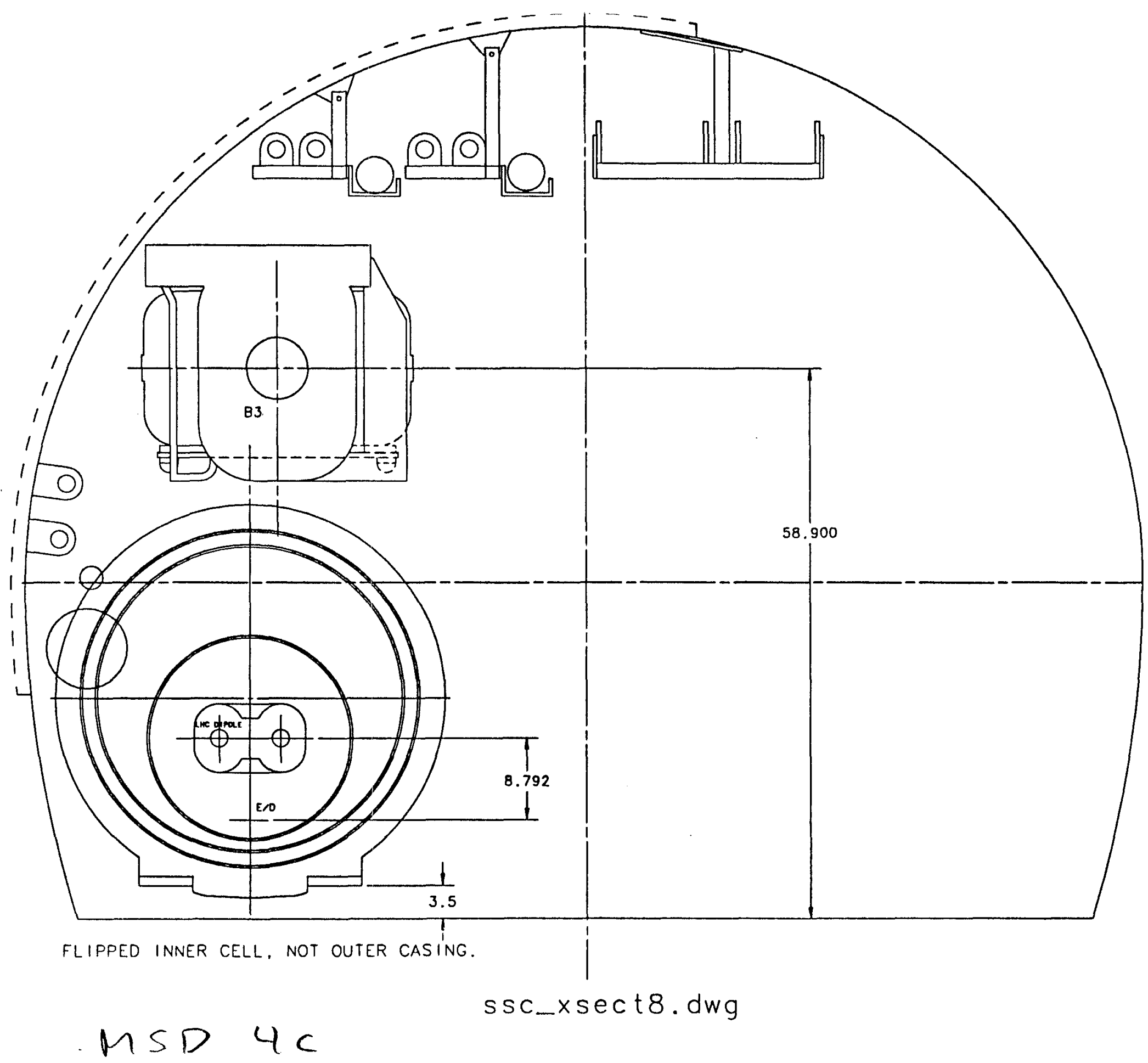




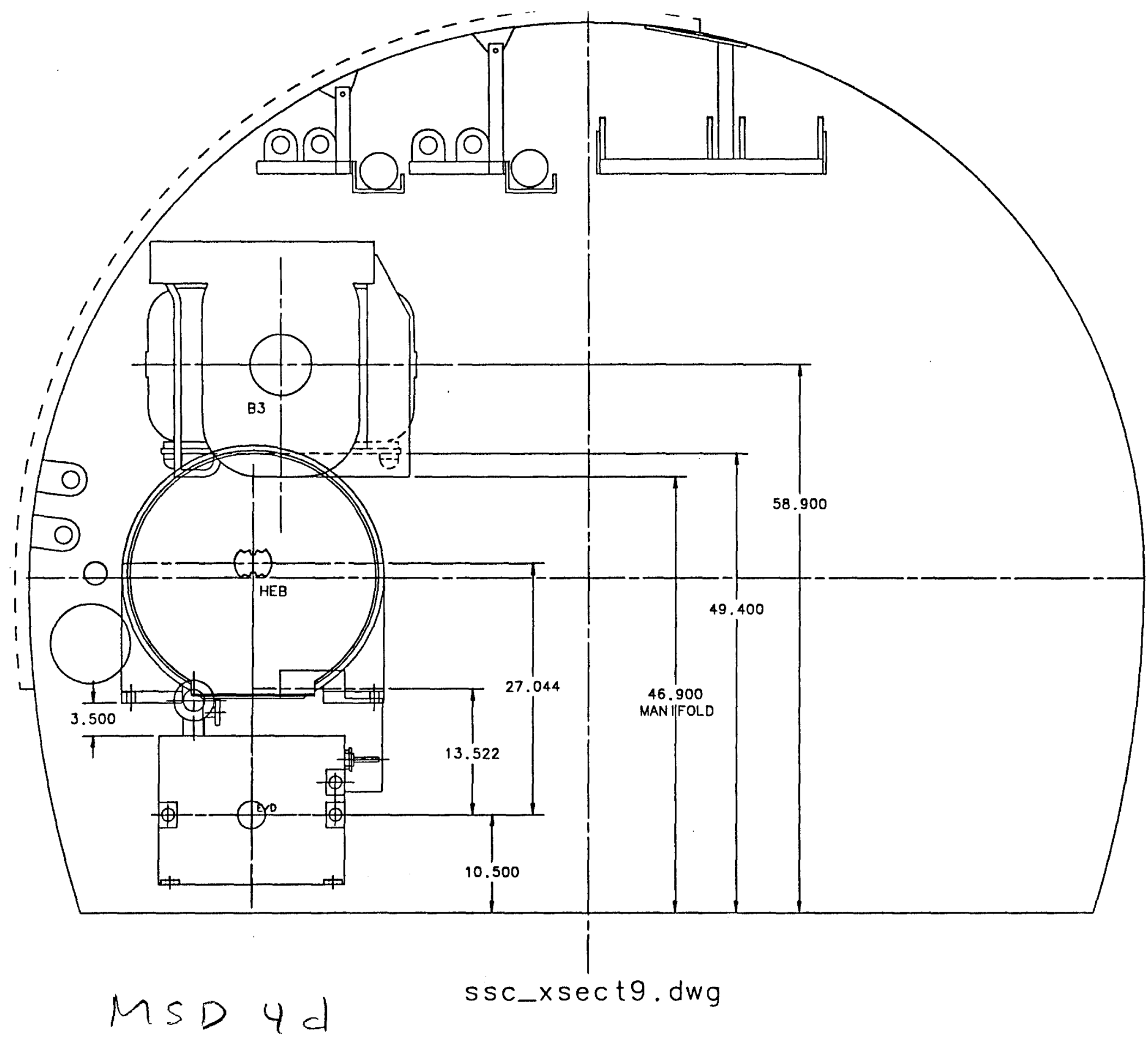




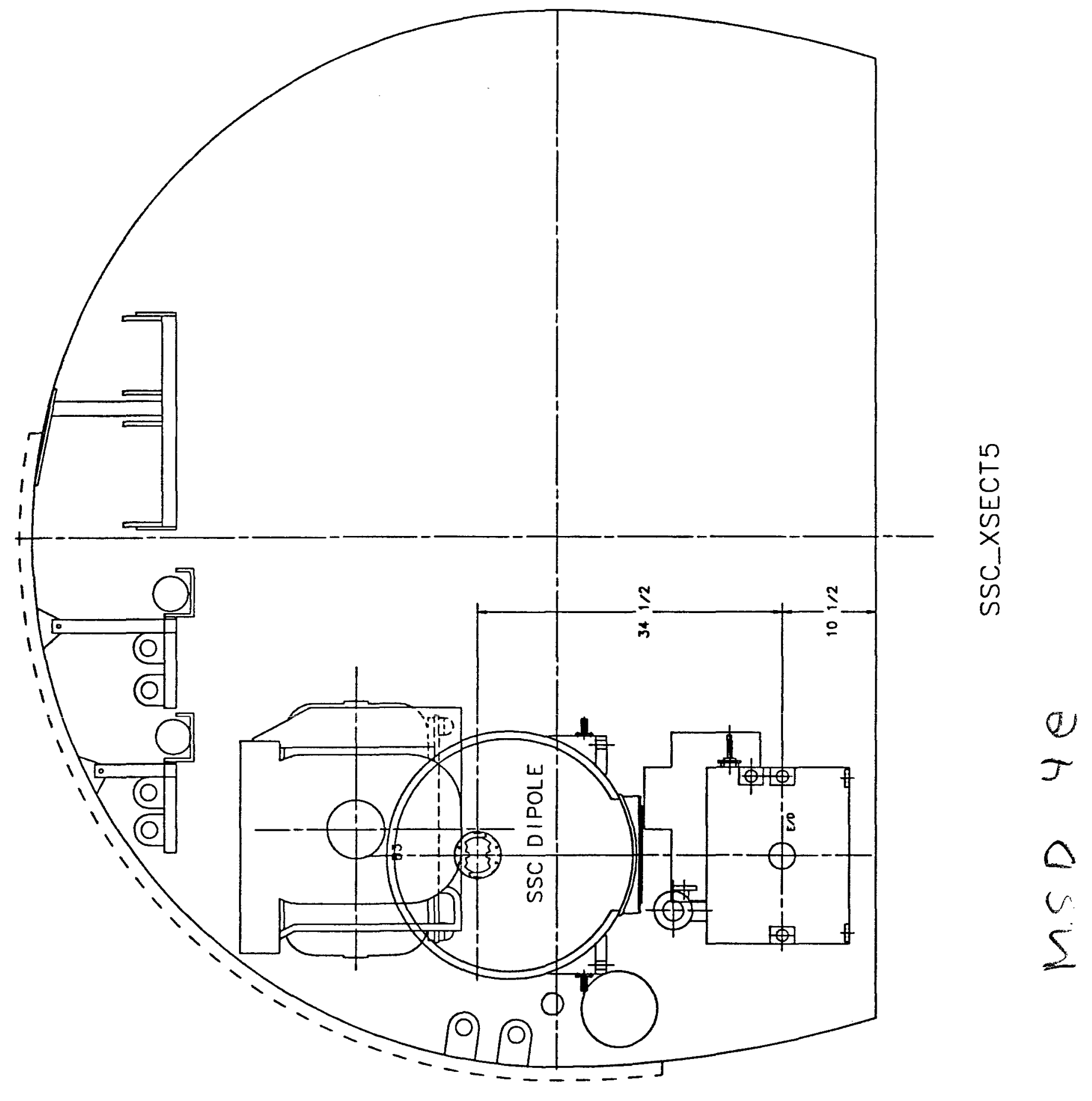




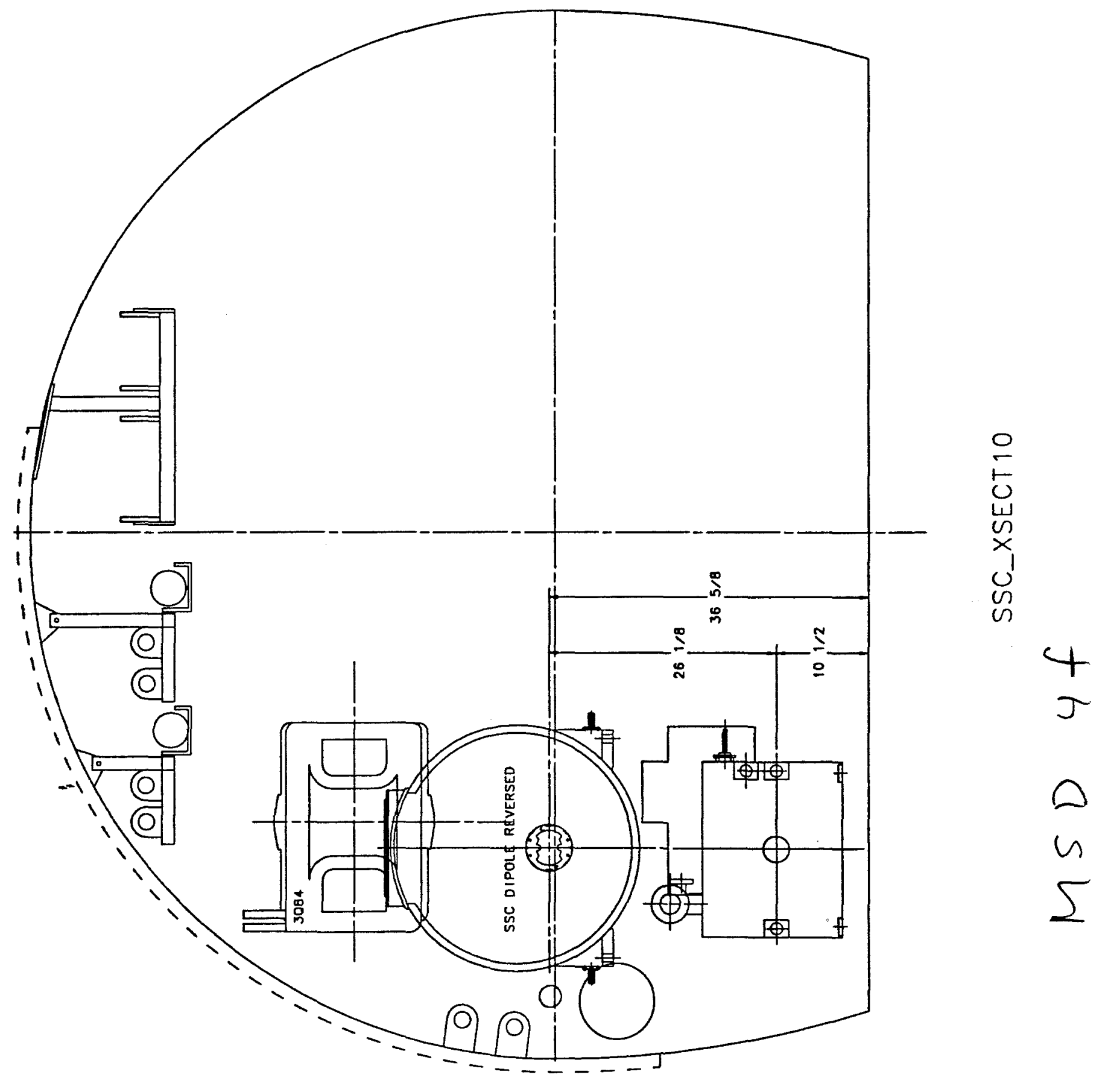




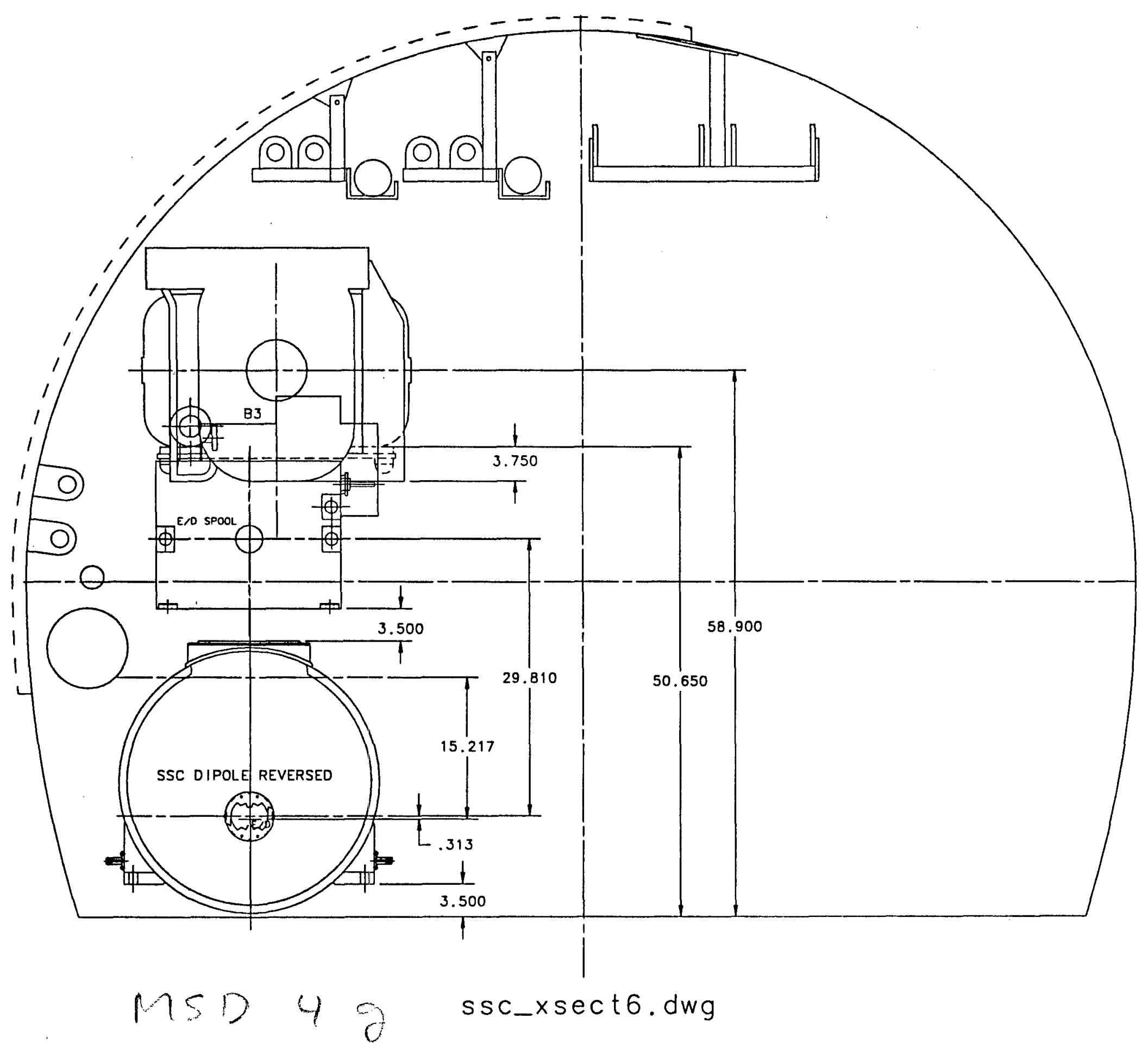




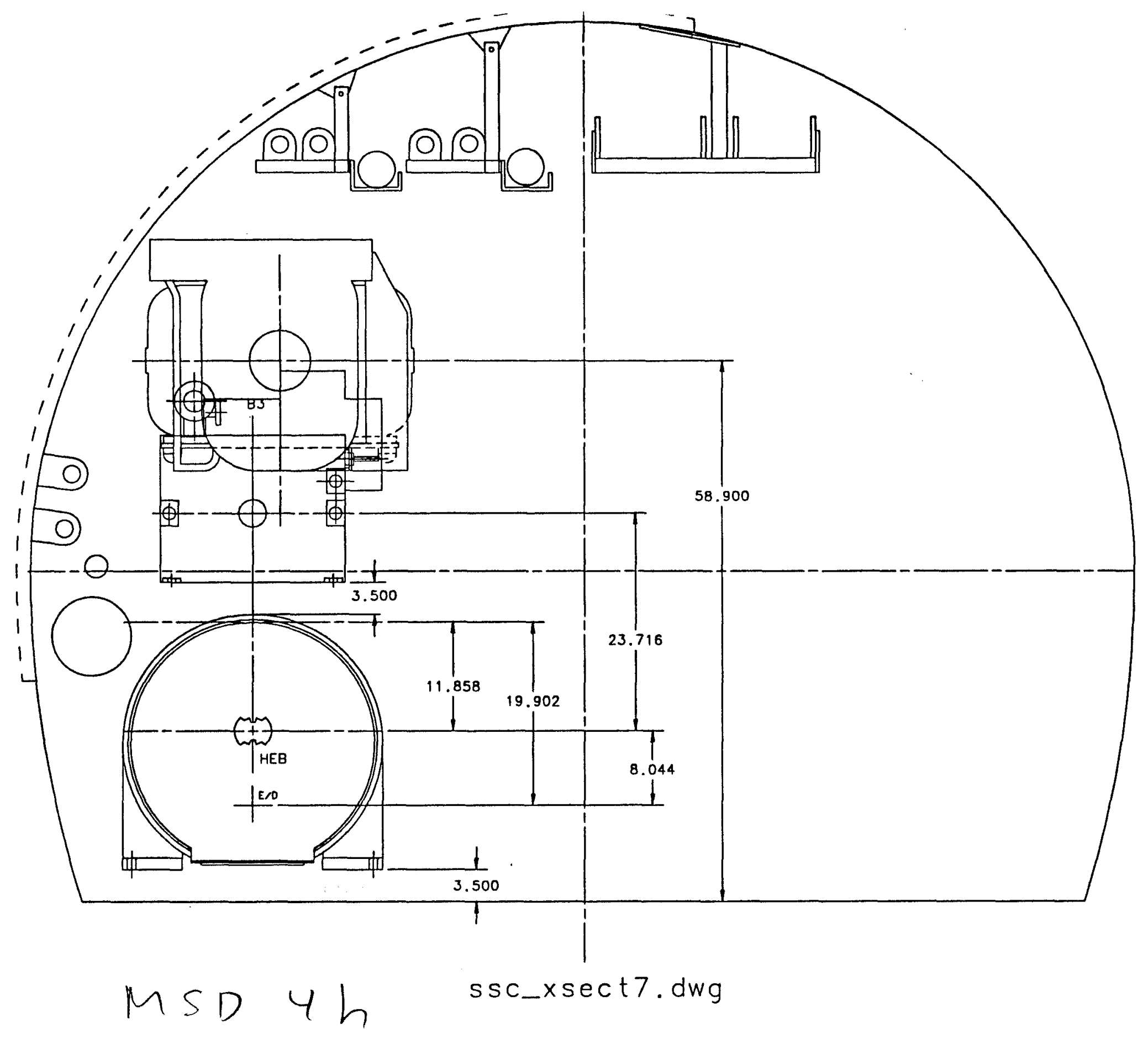




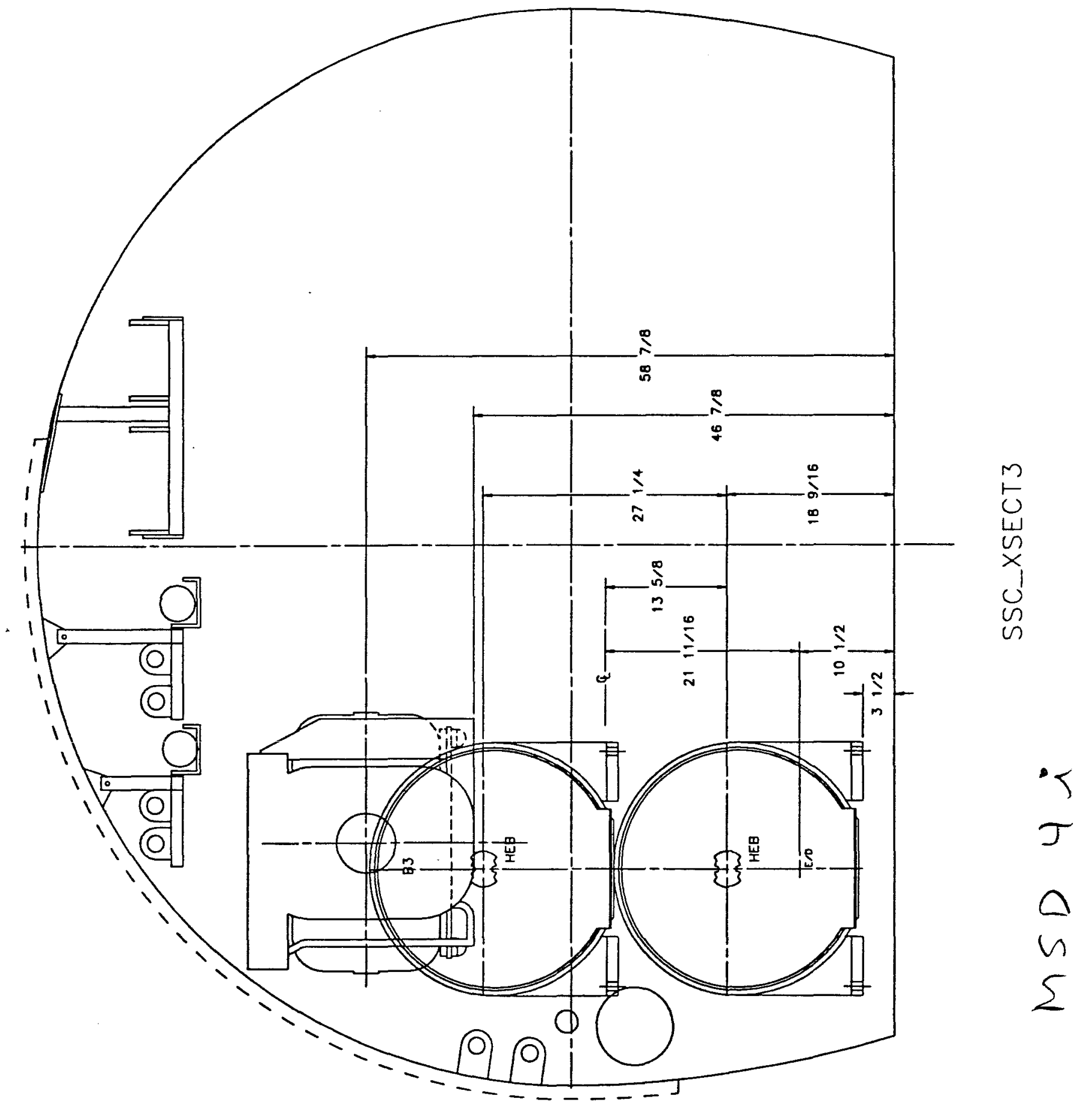




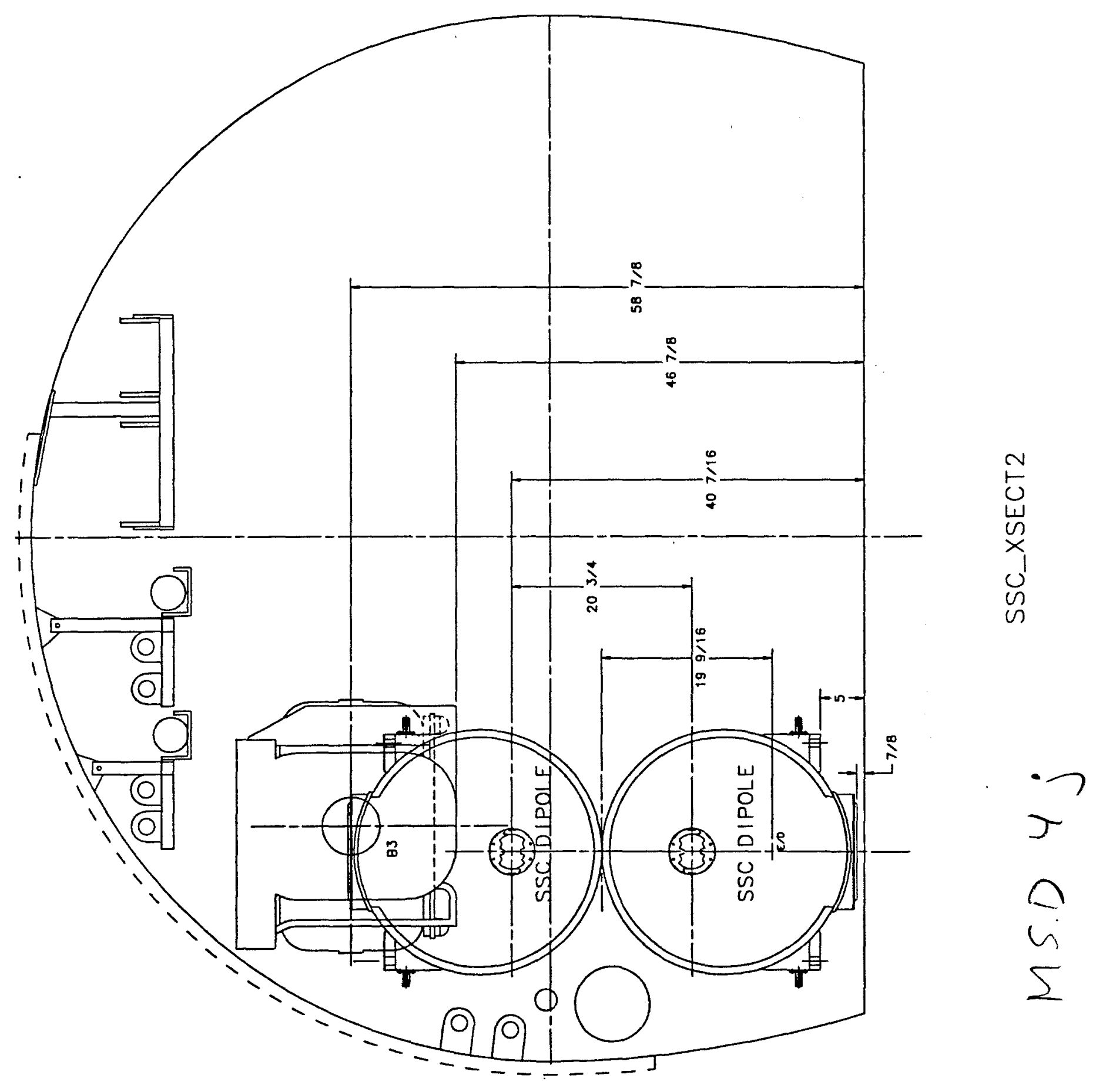


DRAFT

December, 1993

\title{
Physics Sensitivity of Fermilab Machine Options
}

\author{
Christopher T. Hill \\ and \\ Stephen J. Parke \\ Fermi National Accelerator Laboratory \\ P.O. Box 500, Batavia, Illinois, 60510
}

\section{(1) Top Quark Factory}

The top quark has proven to be much heavier than originally anticipated and is now the only expected sequential fermion with a mass of order the weak symmetry breaking scale itself. This suggests that the top quark is strongly coupled, through new physics, to the dynamics of electroweak symmetry breaking. There are at least three ways in which new physics can begin to make an appearance through top:

(i) oblique radiative corrections as in the comparison of $m_{t}$ to $M_{W}$ or $\sin ^{2} \theta$, etc., may yield inconsistencies when subject to high statistics precision tests;

(ii) the search for exotic decay modes, such as $t \rightarrow H^{+}+b$, may yield the first observation of scalars as in multiple Higgs schemes or some of the plethora of SUSY particles; other modes, such as $t \rightarrow \gamma c$ or $t \rightarrow Z c$, if observed, would be indicative of new physics;

(iii) the production distributions of top are sensitive to exotic intermediate heavy states which may favor strong coupling to the top quark.

The sensitive comparison of $M_{W}$ to $m_{t}$ will require determination of $M_{W}$ to a precision of order $\pm 30 \mathrm{MeV}$ and $m_{t}$ to better than $\pm 10 \mathrm{GeV}$. This can 
occur completely within the context of the Fermilab program. The resulting consistency check on the standard model is shown in Fig.(1). We show here a result that would confirm the standard model; there is the possibility of generating here $\mathrm{a} \sim 3 \sigma$ discrepancy with the standard model. Nonstandard top decays within the context of multi-Higgs boson generalizations of the standard model, which include SUSY theories, are expected at branching ratios of order $\sim 10^{-3}$ to $10^{-2}$, as shown for the most recent SUSY limits in Fig.(2) from ref.[2]. In these models the top quark acts as a catalyst in the production of charged Higgs bosons. Finally, the $t \bar{t}$ system can be used as a Drell-Yan pair (i.e., as a substitute for $\mu \bar{\mu}$ ) to probe new physics in the $p_{T}$ production distributions of the top quark and decay product $W$ boson, as in an example case of ref.[1] shown in Fig.(3).

This suggests that a fruitful near-term physics goal would consist of upgrades of the Fermilab Tevatron/Main Injector program in luminosity or energy to act effectively as a top quark factory. We arbitrarily prescribe an asymptotic goal of $10^{6}$ produced top quarks. We assume the upgrade options detailed by D. Finley, and give the top production rates, per week, versus top quark mass in Figs.(4) and (5).

\section{(2) Higgs Bosons}

There is a mass range for the standard model Higgs boson which is difficult for any machine to explore, and in which a $p \bar{p}$ machine has a decided advantage. This extends roughly from $m_{H}=60 \mathrm{GeV}$ (the present lower limit) to $m_{H} \approx$ $200 \mathrm{GeV}$. The Higgs boson is produced most copiously through gluon fusion, Fig.(5), however the backgrounds to the $H \rightarrow b \bar{b}$ or $H \rightarrow W+W^{*}$ decay modes are overwhelming at energies below the SSC. However, associated production of Higgs together with a $W$ boson in a $p \bar{p}$ machine is only an order of magnitude below gluon fusion at $\sqrt{s}=2 \mathrm{TeV}$, and provides a very powerful tag and background suppression to the detection of the Higgs. This proceeds through the virtual $W$ diagram of Fig.(5), which involves no small Higgs-Yukawa couplings. The mass range probed here is interesting because the decay modes of Higgs depend sensitively upon the opening of the $W W$ final state. The discovery potential for this mode is driven by the total integrated luminosity.

We have rescaled the yields from Ref.[3], for $10 \mathrm{fb}^{-1}$ with their acceptance cuts (all at $\sqrt{s}=2 \mathrm{TeV}$ ): 


\begin{tabular}{||l|c|c|c||}
\hline$M_{H}(\mathrm{GeV})$ & $W H / Z H$ & $W b \bar{b} / Z b \bar{b}$ & $N \sigma$ \\
\hline \hline 60 & $500 / 580$ & $600 / 520$ & $20 \sigma$ \\
\hline 80 & $280 / 350$ & $390 / 380$ & $14 \sigma$ \\
\hline 100 & $150 / 200$ & $250 / 260$ & $9 \sigma$ \\
\hline 120 & $80 / 100$ & $170 / 180$ & $6 \sigma$ \\
\hline 140 & $30 / 38$ & $110 / 130$ & $3 \sigma$ \\
\hline \hline
\end{tabular}

If $M_{H}>140 \mathrm{GeV}$, then we are interested in the production of the states $(W, Z \rightarrow \ell \ell)+\left(H \rightarrow W W^{*}\right)$. Isolated like-sign dileptons with missing $p_{T}$ have no significant background. We expect for $m_{H}=200 \mathrm{GeV} \sim 300$ produced Higgs boson and $\sim 15$ like-sign dilepton events (backgroundless) of this type for $10 \mathrm{fb}^{-1}$.

\section{(3) B-Physics}

Hadron colliders offer many clear advantages to $B$-physics studies relative to $e^{+} e^{-}$machines. The key reasons are (i) the $B$ production cross sections are very large, typically $50 \mu b$ at Tevatron (vs. $10 n b$ at the SLAC asymmetric $\mathrm{B}$ factory) and (ii) the beam is broad-band, ergo all $B$ system states are produced. $e^{+} e^{-}$machines typically sit upon the $\Upsilon(4 S)$. This offers the advantage of kinematic reconstruction since the initial $B$ energy is known (which can be achieved at hadron machines by other means). However, in a hadron machine one makes the following states in addition to the conventional $B_{u}$ and $B_{d}$ (the following are unavailable at an $e^{+} e^{-} \Upsilon(4 S)$ machine, such as the SLAC asymmetric $B$-factory): $B_{s}, B_{c}$, single heavy $b$-baryons, double-heavy baryons, triple heavy, all $B$-system mesonic and baryonic resonances. The resonances are particularly interesting because of their tight interplay with chiral symmetry in QCD and as a method of providing charged pions and Kaons for flavor tagging of daughter states. CP-violation in the $B$ system is readily observable in this way, and could be discovered in principle at the Tevatron in run II at CDF. 


\begin{tabular}{||l|c|c|c|c||}
\hline Mode & Tevatron $^{(a)}$ & Main Injector $^{(b)}$ & SLAC ABF $^{(c)}$ & LEP II $^{(d)}$ \\
\hline \hline$B_{u, d}$ & $3 \times 10^{9}$ & $3 \times 10^{10}$ & $3 \times 10^{8}$ & $3 \times 10^{6}$ \\
\hline$B_{s}$ & $0.5 \times 10^{9}$ & $0.5 \times 10^{10}$ & none & $10^{6}$ \\
\hline$B_{c}$ & $3 \times 10^{7}$ & $3 \times 10^{8}$ & none & $10^{5}$ \\
\hline$b q q$ & $3 \times 10^{7}$ & $3 \times 10^{8}$ & none & $10^{5}$ \\
\hline$b b q$ & $3 \times 10^{4}$ & $3 \times 10^{5}$ & none & $10^{2}$ \\
\hline$b b b$ & 30 & 300 & none & none \\
\hline
\end{tabular}

Table 1: Indicated yields of some usable $b$ quark containing hadrons, running for a 3 year, $30 \%$ duty cycle, period for: (a) Tevatron at present attainable $\mathcal{L}=10^{31} \mathrm{~cm}^{-2} \mathrm{sec}^{-1}$ (b) Main Injector assuming $\mathcal{L}=10^{32} \mathrm{~cm}^{-2} \mathrm{sec}^{-1}$ (twice the design goal); multiply by 10 if the rapidity range is $|\eta| \leq 3$ and $p_{t}>5 \mathrm{GeV}$. (c) $A B F-A s y m-$ metric B-factory proposal at $\mathcal{L}=10^{34} \mathrm{~cm}^{-2} \mathrm{sec}^{-1}$ operating on the $\Upsilon(4 S)$ (d) LEP at $Z^{0}$-pole with $\mathcal{L}=2 \times 10^{31} \mathrm{~cm}^{-2} \mathrm{sec}^{-1}$. 


\section{Figure Captions}

Fig.(1) Electroweak radiative corrections allow comparison of $m_{t}$ to $M_{W}$ for various values of the S.M. Higgs boson mass. We superimpose the result of a $\pm 30 \mathrm{MeV}$ determination of $M_{W}$ and a $\pm 10 \mathrm{GeV}$ determination of $m_{t}$.

Fig.(2) Nonstandard decays of top in multi-Higgs boson generalizations, which include the minimal supersymmetric standard model, are expected at the $\sim 10^{-2}$ level of branching ratio, from ref.[2].

Fig.(3) Nonstandard production including the coherent effects of a color octet heavy resonance (e.g. a "topgluon") are shown for a resonance mass of 0.6 $\mathrm{TeV}, 0.8 \mathrm{TeV}, 1.0 \mathrm{TeV}$, and pure QCD (infinite resonance mass), from ref.[1].

Fig.(4) Weekly top production rates vs. $m_{t}$ for the various $p \bar{p}$ machine options.

Fig.(5) Weekly top production rates vs. $m_{t}$ for the various $p p$ machine options.

Fig.(6) Cross-sections for Higgs production from ref.[3]. Dominant gluon fusion cross-section is swamped by backgrounds. Associated $W+H$ and $Z+H$ are indicated, for the Tevatron. The diagram for associated $W+H$ production is indicated.

Fig.(7) Branching ratios for Higgs decay vs mass of Higgs boson from ref.[3].

Fig.(8) Weekly rates for associated Higgs production with the $W$ boson vs $M_{H}$ for various machine options ( $p \bar{p}$ only).

Fig.(9) Weekly rates for associated Higgs production with the $Z$ boson vs $M_{H}$ for various machine options ( $p \bar{p}$ only).

\section{References}

[1] C. T. Hill and S. Parke, in preparation; C. T. Hill, Phys. Lett. B266 419 (1991).

[2] F. Borzumatti, Desy Preprint, Desy-93-99, (1993)

[3] A. Stange, W. Marciano, S. Willenbrock "Higgs Bosons at the Fermilab Tevatron," Fermilab-Pub-93/142-T 


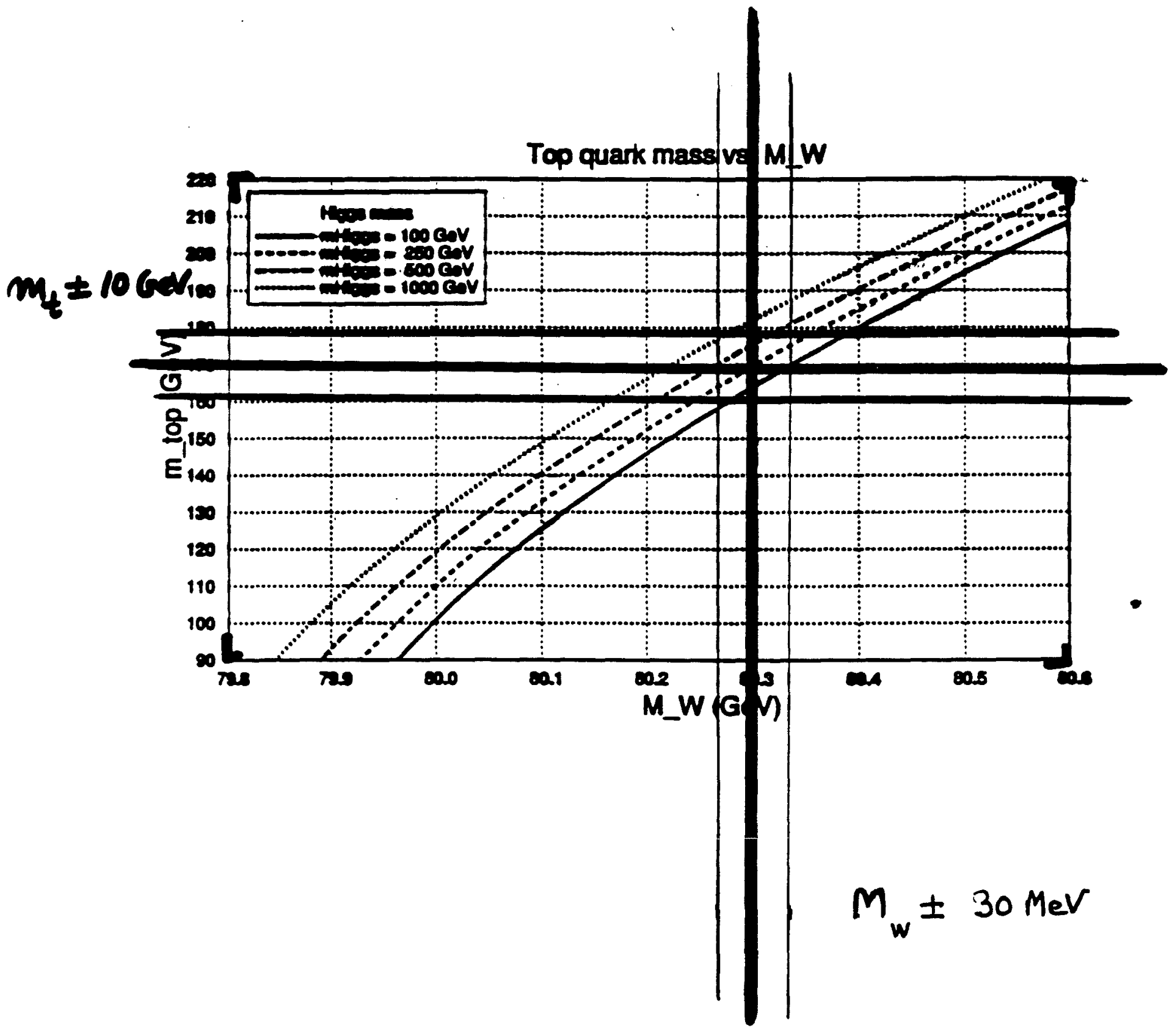

Physics Sensitivity.. 
F. Borzumatti (Desy-93-99)

Top as Chaged Higs fietory Mssm

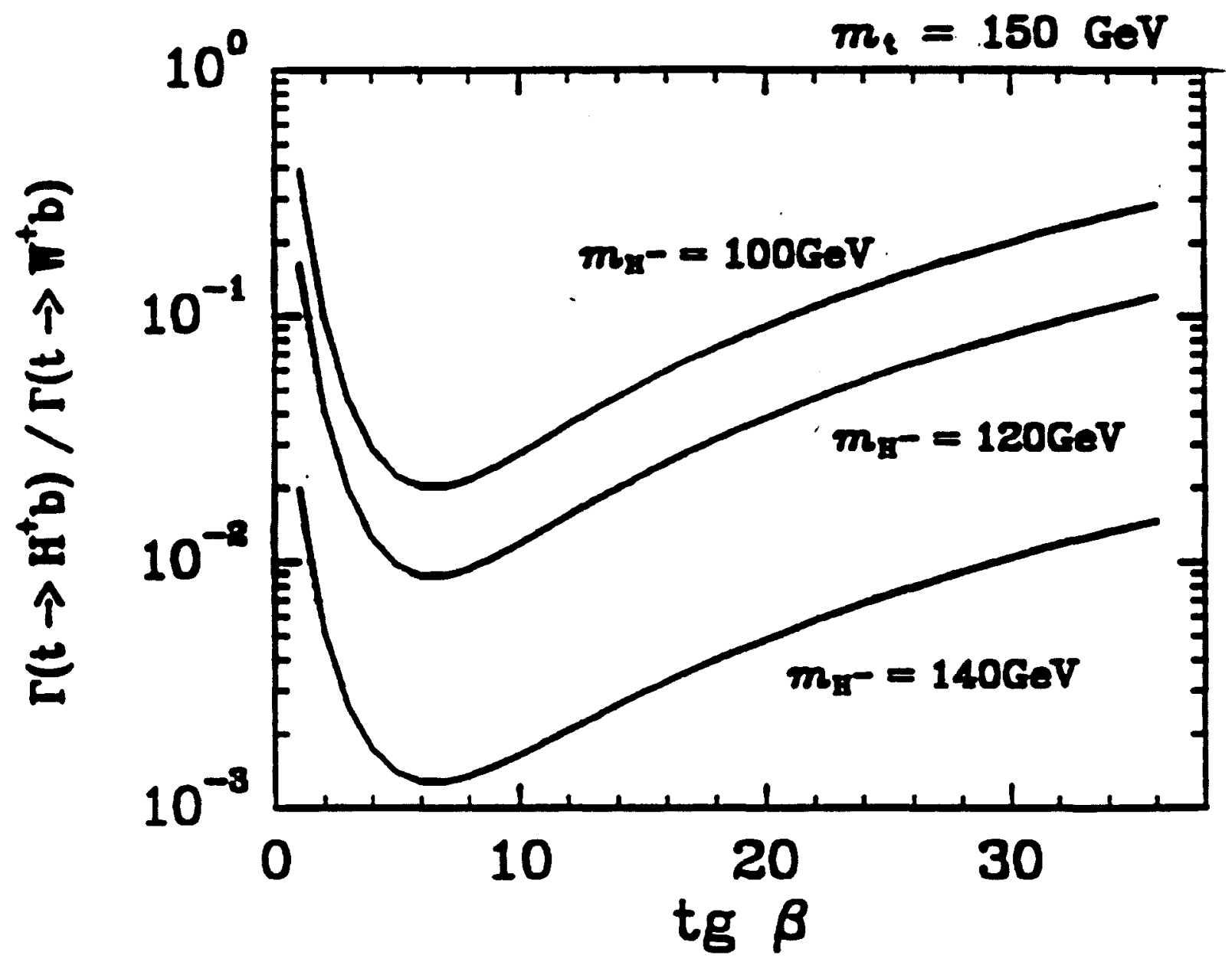

$$
\begin{aligned}
& m_{\text {f }}>120 \mathrm{GeV}, \quad m_{\tilde{d}_{1}, \tilde{u}_{2}}>100 \mathrm{GeV}, \quad m_{\tilde{u}_{1}}>45 \mathrm{GeV} \text {, } \\
& m_{\tilde{x}_{2}^{-}}>45 \mathrm{GeV}, \quad m_{\nu_{1}}>45 \mathrm{GeV}, \quad m_{\tilde{\jmath}_{1}}>45 \mathrm{GeV} \text {, } \\
& m_{h_{2}^{\circ}}>30 \mathrm{GeV}, \quad m_{\tilde{x}_{i}^{0}}>20 \mathrm{GeV} \text {. }
\end{aligned}
$$




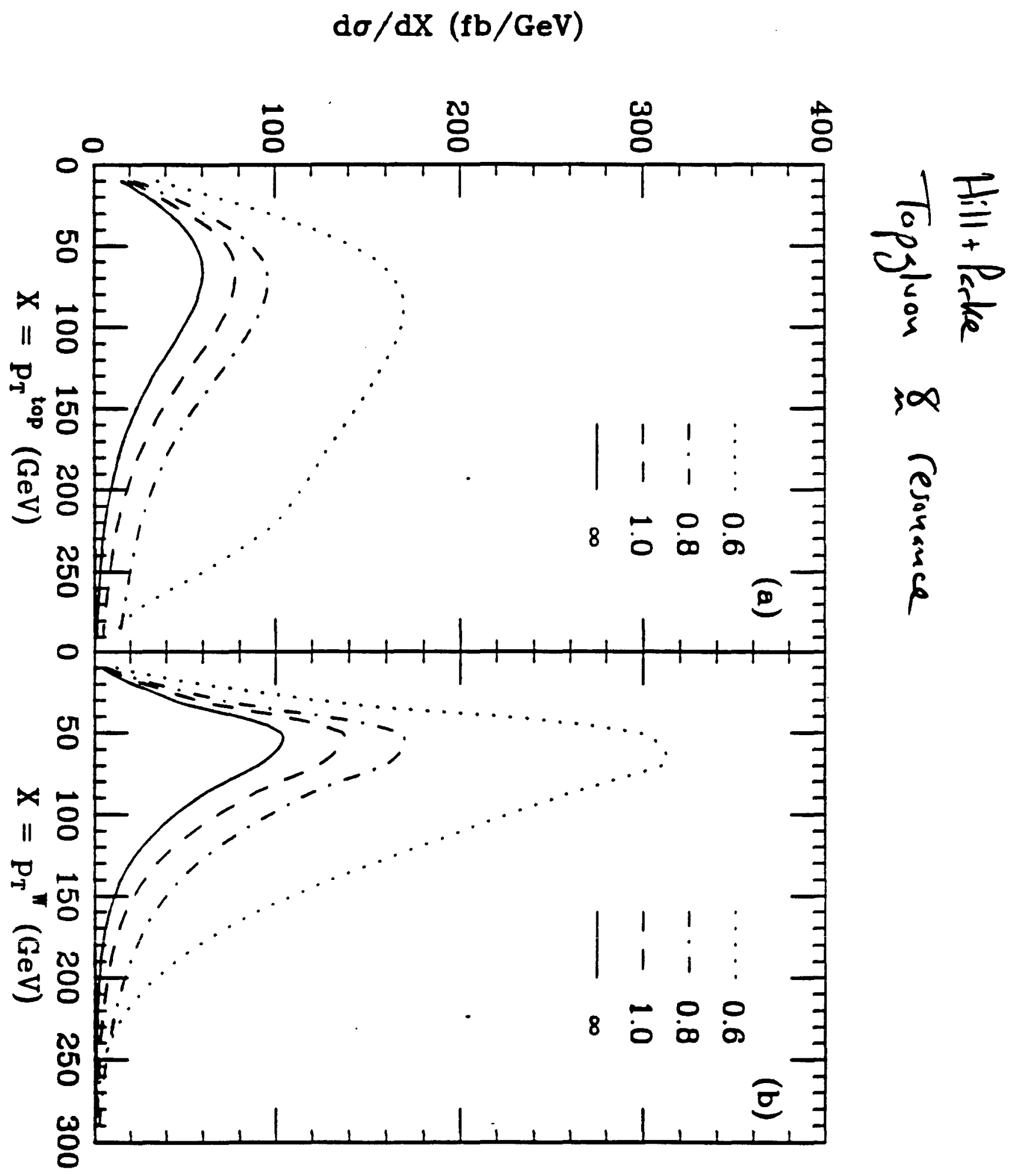




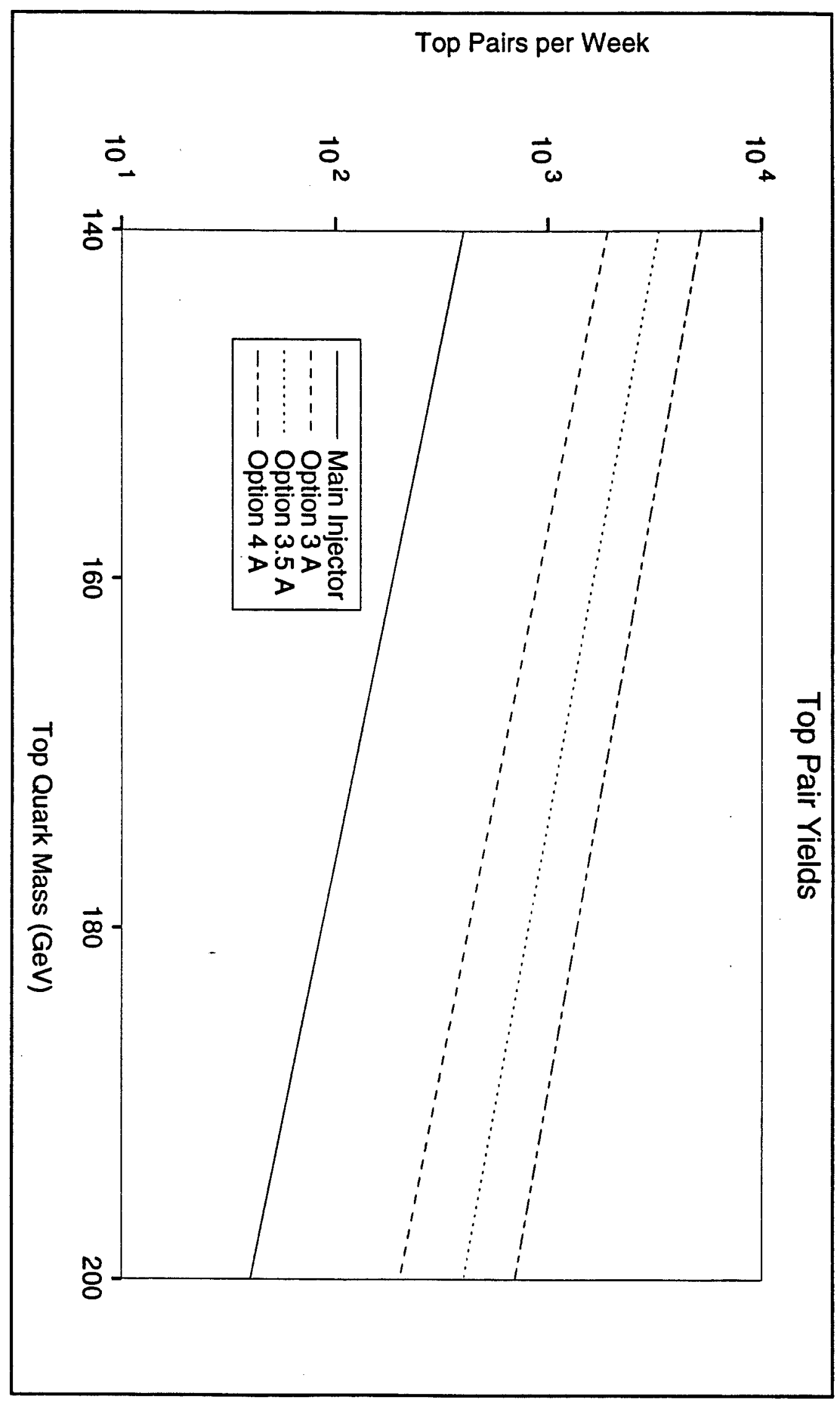

Physics Sensitivity... 


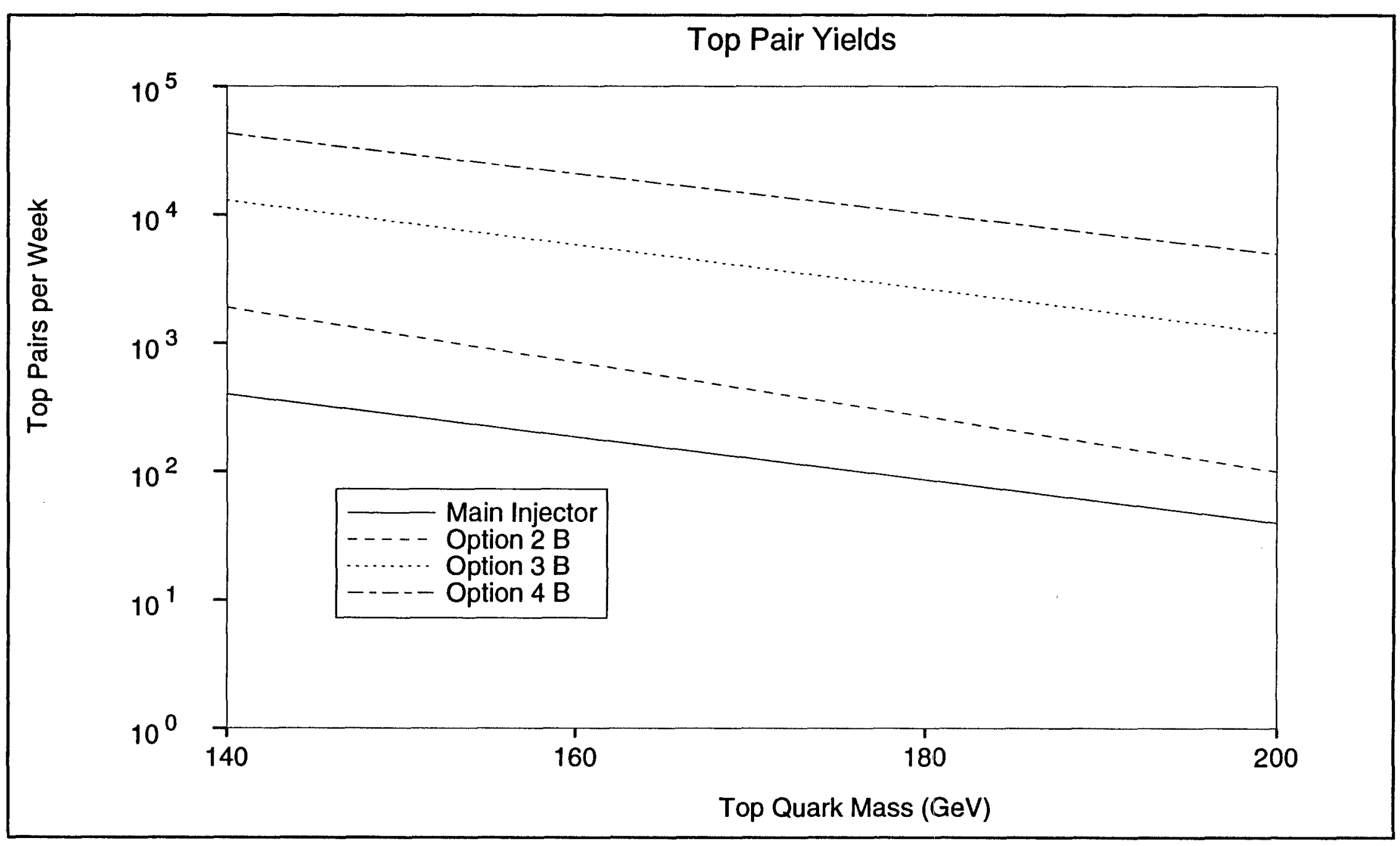


Stany, Mociame, Willenbrede

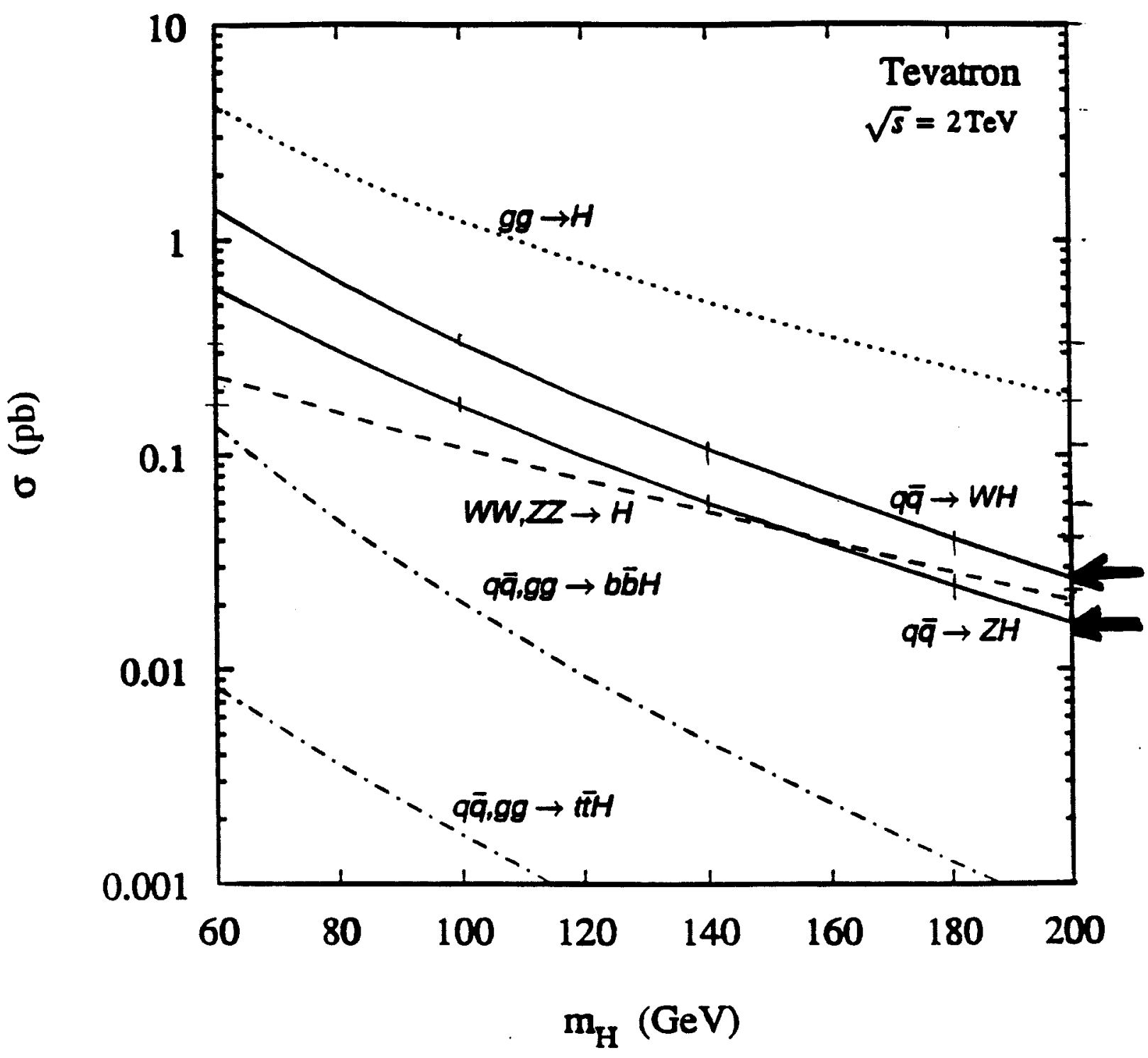

$q \bar{q} \rightarrow W H^{\circ}$

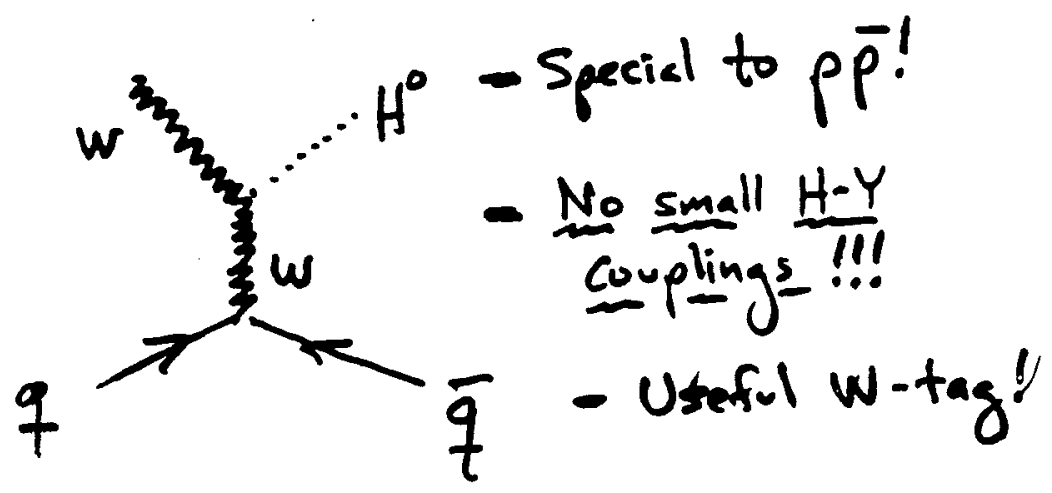


Staigy, Merriang willew soren

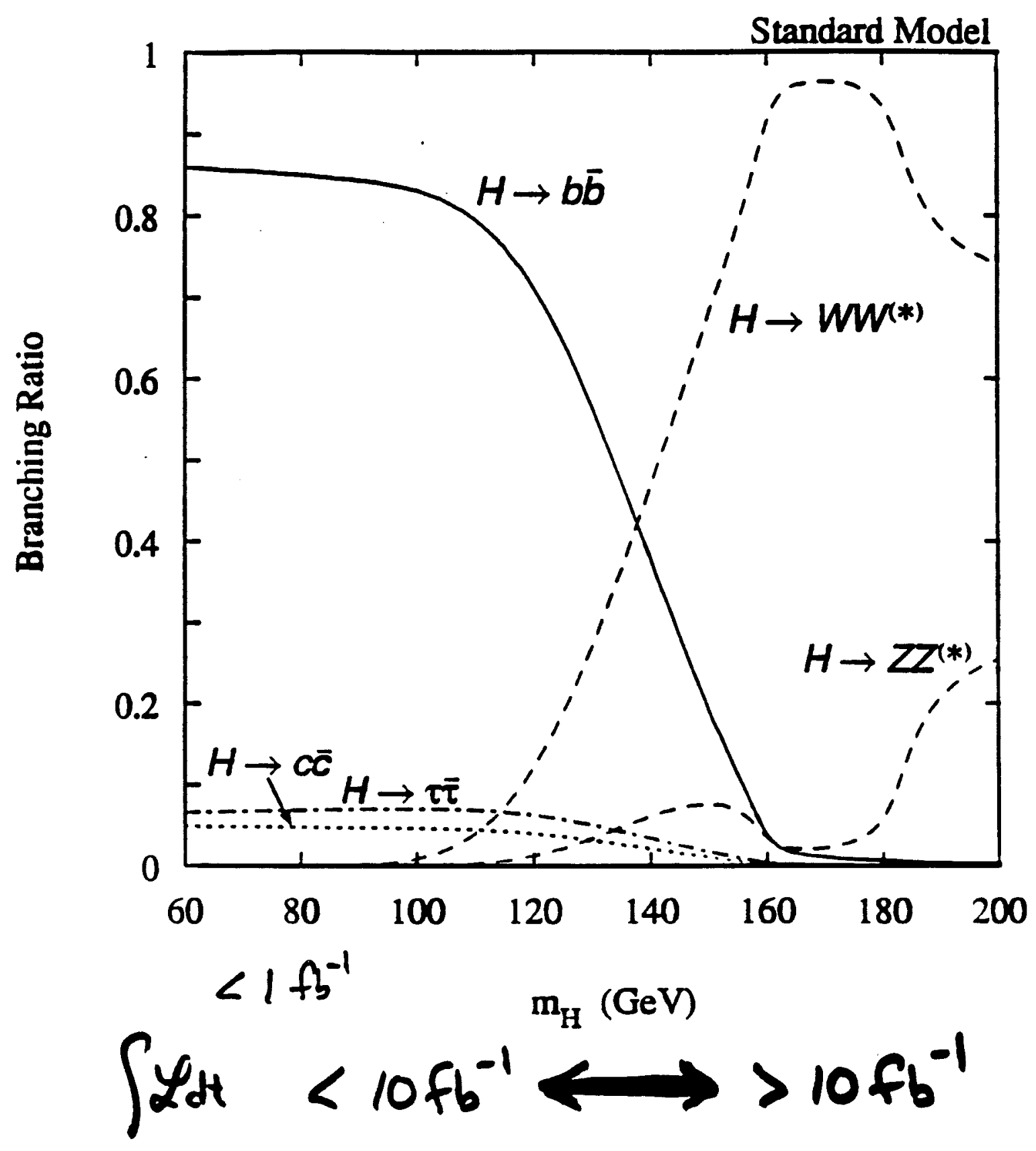

Physics Sensitivity... 


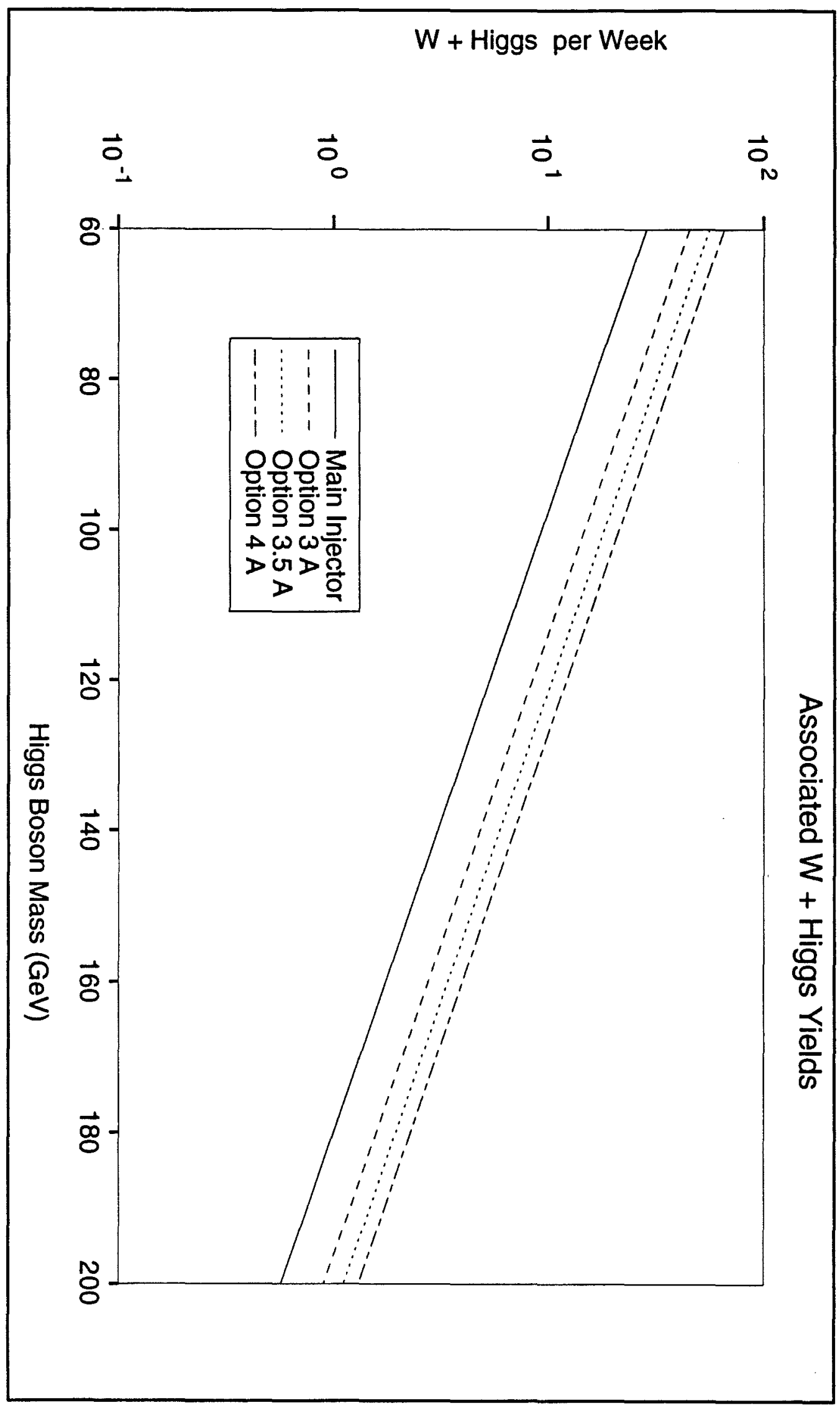

Physics Sensitivity... 


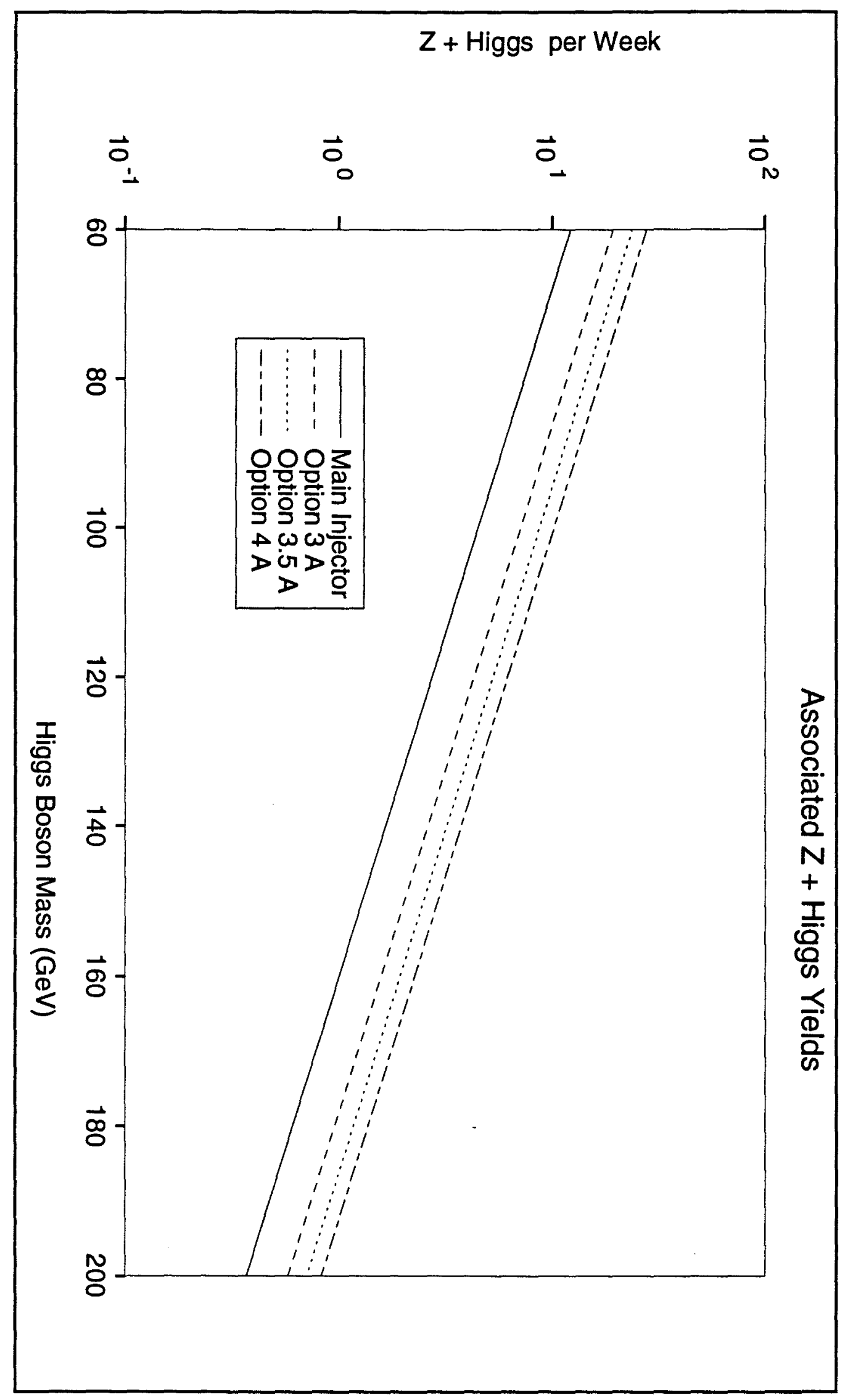

Physics Sensitivity... 

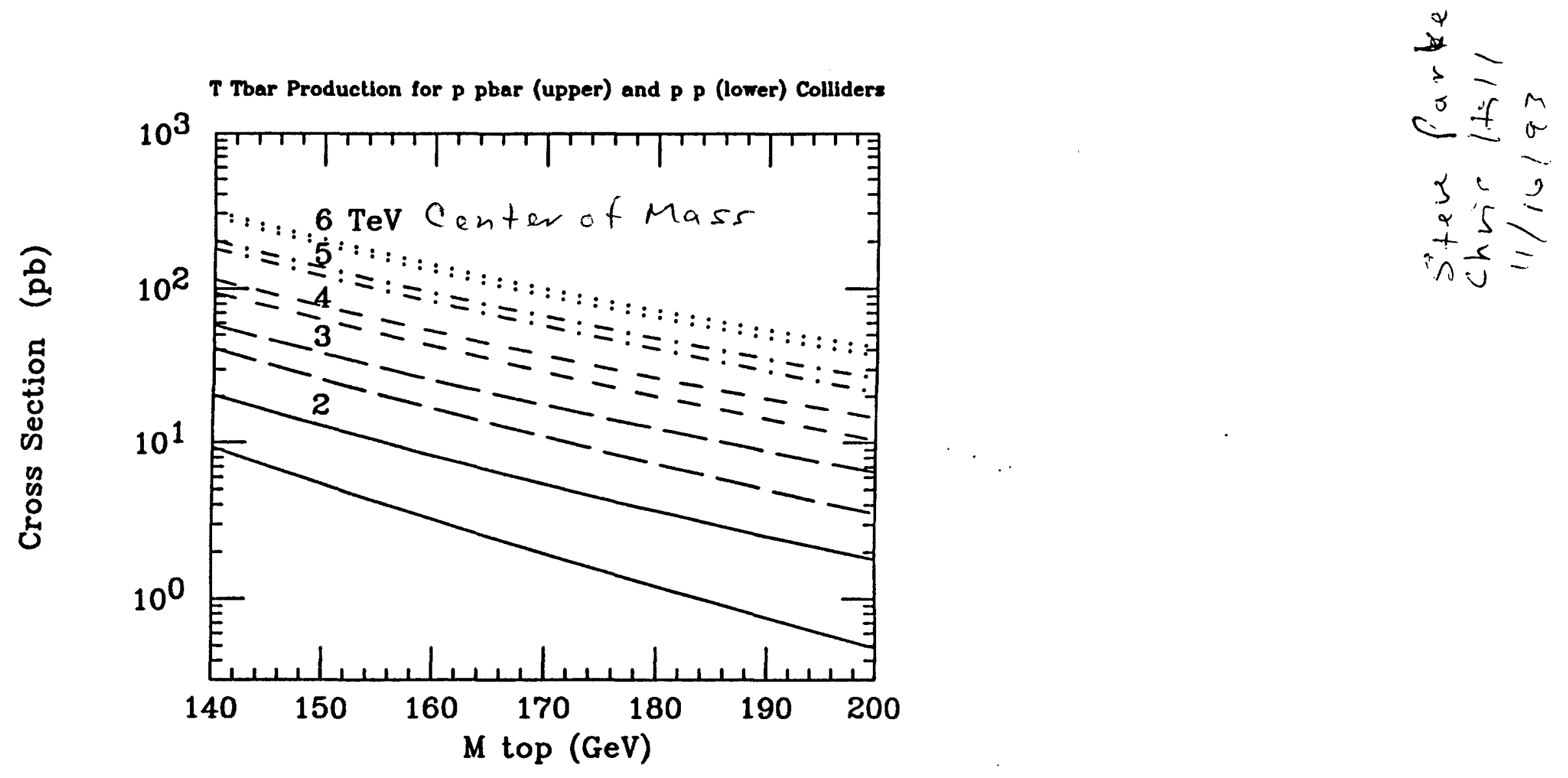

PH 